\title{
Towards basin-scale in-situ characterization of sea-ice using an Autonomous Underwater Glider
}

\author{
by \\ Zachary Duguid
}

S.B. Aerospace Engineering, Massachusetts Institute of Technology (2018)

Submitted to the Department of Mechanical Engineering and to the Joint Program in Applied Ocean Science and Engineering in partial fulfillment of the requirements for the degree of

Master of Science in Mechanical Engineering

at the

MASSACHUSETTS INSTITUTE OF TECHNOLOGY

and the

WOODS HOLE OCEANOGRAPHIC INSTITUTION

September 2020

(c) Zachary Duguid 2020. All rights reserved.

The author hereby grants to MIT and WHOI permission to reproduce and to distribute publicly paper and electronic copies of this thesis document in whole or in part in any medium now known or hereafter created.

Author

Zachary Duguid

Joint Program in Applied Ocean Science and Engineering

August 7th, 2020

Certified by

Richard Camilli

Woods Hole Oceanographic Institution

Thesis Supervisor

Accepted by

David Ralston

Woods Hole Oceanographic Institution

Chair, Joint Committee for Applied Ocean Science and Engineering

Accepted by

Nicolas Hadjiconstantinou

Massachusetts Institute of Technology

Chair, Department Committee on Graduate Theses 


\title{
Towards basin-scale in-situ characterization of sea-ice using an Autonomous Underwater Glider
}

\author{
by \\ Zachary Duguid
}

Submitted to the Department of Mechanical Engineering and to the Joint Program in Applied Ocean Science and Engineering in partial fulfillment of the requirements for the degree of Master of Science in Mechanical Engineering

\begin{abstract}
This thesis presents an Autonomous Underwater Glider (AUG) architecture that is intended for basin-scale unattended survey of Arctic sea-ice. The distinguishing challenge for AUG operations in the Arctic environment is the presence of year-round sea-ice cover which prevents vehicle surfacing for localization updates and shore-side communication. Due to the high cost of operating support vessels in the Arctic, the proposed AUG architecture minimizes external infrastructure requirements to brief and infrequent satellite updates on the order of once per day. This is possible by employing onboard acoustic sensing for sea-ice observation and navigation, along with intelligent management of onboard resources.

To enable unattended survey of Arctic sea-ice with an AUG, this thesis proposes a hierarchical acoustics-based sea-ice characterization scheme to perform science data collection and assess environment risk, a multi-factor terrain-aided navigation method that leverages bathymetric features and active ocean current sensing to limit localization error, and a set of energy-optimal propulsive and hotel policies that react to evolving environmental conditions to improve AUG endurance. These methods are evaluated with respect to laboratory experiments and preliminary field data, and future Arctic sea-ice survey mission concepts are discussed.
\end{abstract}

Thesis Supervisor: Richard Camilli

Title: Associate Scientist with Tenure 


\section{Acknowledgments}

First, I would like to extend my deepest gratitude to Richard Camilli for believing in me as a researcher and for inspiring me to "dream big." Thank you for mentoring me and advocating on my behalf throughout my graduate program.

Next, I express my sincere thanks to my colleagues at the Deep Submergence Lab for guiding me along the way, especially Angelos Mallios, Al Bradley, Brian Claus, Oscar Pizarro, Greg Burgess, Peter Ventola, Judy Fenwick, and Heather Marin. Special thanks to Judy for her helpful editing suggestions on this thesis.

For inviting me to participate in my first sea-ice field work experience and for teaching me proper ice canoeing form, I thank Dany Dumont, Peter Sutherland, Luc Barast, Hugo Sellet, Seth Zippel, and Ted Maksym.

For orchestrating the research deployment at the Kolumbo Volcano, I thank Paraskevi Nomikou, Angelos Mallios, Richard Camilli, the MIT MERS group, the 2019 Kolumbo Expedition Science Team, the captain and crew of the CLV/ Ocean Link, and the Greek Ministry of Foreign Affairs.

I gratefully acknowledge the faculty and staff at both MIT and WHOI for their advice and support during my graduate program, especially John Leonard, Brian Williams, Pierre Lermusiaux, Mike Benjamin, Leslie Regan, Beth Marois, Kris Kipp, Andone Lavery, and Gordon Zhang. Additionally, I thank my fellow Joint Program and MechE students for their friendship.

Lastly, I thank my friends and family for supporting me and for cheering me up whenever I needed it. I especially thank my parents, Rebecca and John, and my sister, Hannah, for their unconditional love, and Annie for being my best friend and support system.

Support for this research was provided through the National Science Foundation Navigating the New Arctic Grant \#1839063 and the NASA PSTAR Grant \#NNX16AL08G. Additionally, this research was supported by the Walter A. Rosenblith Presidential Fellowship. 
This page intentionally left blank. 


\section{Contents}

$\begin{array}{ll}\text { List of Figures } & 11\end{array}$

$\begin{array}{ll}\text { List of Tables } & 19\end{array}$

$\begin{array}{ll}\text { Nomenclature } & 23\end{array}$

1 Introduction $\quad 29$

1.1 Motivation for Studying Arctic Sea-ice . . . . . . . . . . . . . . 29

1.2 Review of Arctic Sea-ice Survey Technologies . . . . . . . . . . . . . . 30

1.3 Need for Static and Dynamic Sea-ice Inventory . . . . . . . . . . . . . 33

1.4 Navigating the New Arctic . . . . . . . . . . . . . . . . 34

1.5 Thesis Contributions . . . . . . . . . . . . . . . . . 34

1.6 Thesis Organization . . . . . . . . . . . . . . . 35

2 Proposed Architecture for Autonomous Underwater Glider 37

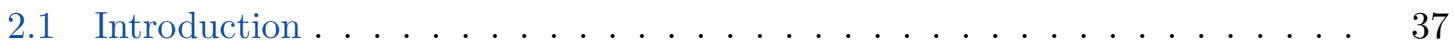

2.2 Review of Hardware Components . . . . . . . . . . . . . . . . . 39

2.3 Hybrid Propulsion . . . . . . . . . . . . . . . . . . . . 40

2.3.1 Buoyancy Engine . . . . . . . . . . . . . . . . . . 41

2.3.2 Propeller Thruster . . . . . . . . . . . . . . . . . . . 42

2.4 Battery Pack Design . . . . . . . . . . . . . . . . . . . 43

2.5 Onboard Acoustic Sensing . . . . . . . . . . . . . . . . 47

2.6 Review of Software Components _ . . . . . . . . . . . . . . . 49

2.7 Risk Aware Planning and Control . . . . . . . . . . . . . . . . 51

2.8 Summary and Future Work . . . . . . . . . . . . . . . . . 52 
3 Synoptic Sea-ice Mapping $\quad 55$

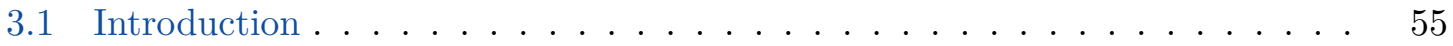

3.2 Properties of Sea-ice . . . . . . . . . . . . . . . . . 56

3.3 Detection of Thin First-year Sea-ice . . . . . . . . . . . . 58

3.4 Hierarchical Characterization of Sea-ice . . . . . . . . . . . . . . 60

3.5 Active Sensing . . . . . . . . . . . . . . . . . 66

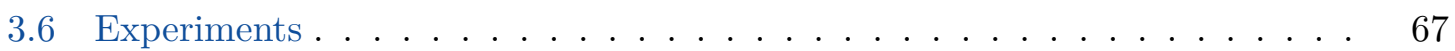

3.6.1 Laboratory Tests in Woods Hole . . . . . . . . . . . . . . 68

3.6.2 Field Deployments in Saint-Fabien . . . . . . . . . . . . . 70

3.7 Results. . . . . . . . . . . . . . . . . . . 73

3.7 .1 Feature Extraction . . . . . . . . . . . . . . . 74

3.7 .2 Sea-ice Detection . . . . . . . . . . . . . . . . . 74

3.8 Summary and Future Work . . . . . . . . . . . . . . . . . . . 79

4 Multi-Factor Terrain-Aided Navigation $\quad 83$

4.1 Introduction . . . . . . . . . . . . . . . . . . 83

4.2 Doppler Velocity Log Odometry . . . . . . . . . . . . . . . . 86

4.2 .1 Dead Reckoning . . . . . . . . . . . . . 87

4.2 .2 Ocean Current Estimation . . . . . . . . . . . . . . . 87

4.3 Multi-Factor Terrain-Aided Navigation . . . . . . . . . . . . . . . 90

4.3.1 Multi-Factor Extraction . . . . . . . . . . . . . . . . . 90

$4.3 .2 \quad$ Particle Filter . . . . . . . . . . . . . . . . . . . . . 92

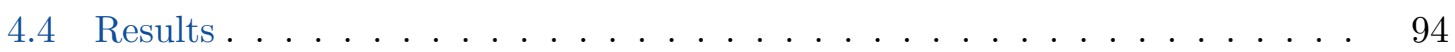

4.4.1 Doppler Velocity Log Odometry . . . . . . . . . . . . . 95

4.4.2 Multi-Factor Terrain-Aided Navigation . . . . . . . . . . . . 96

4.5 Summary and Future Work . . . . . . . . . . . . . . . . . 103

5 Energy-Optimal Control Policies $\quad 105$

5.1 Introduction . . . . . . . . . . . . . . . . . 105

5.2 Objective Function: Minimize Transport Cost . . . . . . . . . . . . . . 105

5.3 Adaptive Velocity Control . . . . . . . . . . . . . . . . . . . . 107

5.3 .1 Simplified 1D Model . . . . . . . . . . . . . . . . . . . . 108

5.3 .2 Generalized 3D Model . . . . . . . . . . . . . . . 111 
5.4 Exploitative Depth Band Selection . . . . . . . . . . . . . . 116

5.5 Adaptive Duty Cycling . . . . . . . . . . . . . . . . . . . . . 118

5.5.1 Satellite Data Transfer . . . . . . . . . . . . . . . . . . . . . . 119

5.6 Results. . . . . . . . . . . . . . . . . . 120

5.6 .1 Adaptive Velocity Control . . . . . . . . . . . . . . . . . . . 121

5.6 .2 Exploitative Depth Band Selection . . . . . . . . . . . . . . . . 122

5.6.3 Mission Concept: Sea-ice Survey at the Chukchi Plateau . . . . . . . 127

5.7 Summary and Future Work . . . . . . . . . . . . . . . . 131

6 Discussion and Future Work 135

6.1 Contributions . . . . . . . . . . . . . . . . . 135

6.2 Future Work . . . . . . . . . . . . . . . . 136




This page intentionally left blank. 


\section{List of Figures}

1-1 Overview of design space for under-ice surveying AUVs and AUGs in terms of mass and range, where the blue highlighted region indicates the area of interest within the design space. The top-right corner of each vehicle image indicates its approximate numerical value within the design space. The scaling of the vehicle images is approximate. . . . . . . . . .

2-1 Rendering of Polarsentinel, the proposed AUG architecture. Polarsentinel is a modified Slocum G3 Electric Glider. Physical modifications include: MSIS for sea-ice characterization, DVL for environment state estimation and navigation, hybrid thruster design for increased efficiency, and updated battery pack for increased energy capacity. . . . . . . . . . . . . . .

2-2 Comparison of transport cost between the buoyancy engine and the prototype thruster when operating in transit mode. The blue solid curves correspond to the transport costs of the buoyancy engine, while the yellow, orange, and red dashed lines correspond with the prototype thruster. . . . . . . . . .

2-3 Battery pack configuration for proposed AUG design. The configuration includes four battery packs: the pitch pack, the payload pack, the aft-short pack and the aft-long pack. Lithium secondary batteries are shown in the diagram, but a similar design is possible for the higher energy density lithium

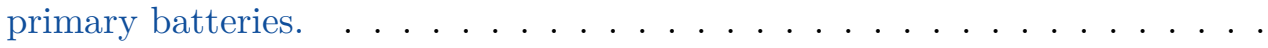


2-4 Subplot A shows the graphical user interface (GUI) that is used for displaying battery state and charging the battery packs when the AUG is connected to a power supply while on the bench. Subplot B shows an example of battery state being tracked over time during a charge sequence, and Subplot $\mathrm{C}$ shows an example of the battery state GUI being used to monitor battery state when the AUG is connected to the bench. . . . . . . . . . . .

2-5 Acoustic beam width for the MSIS and DVL instruments. The MSIS beam width is given by two angles: $\varphi_{M S I S, h}=3^{\circ}$ is the horizontal beam width, and $\varphi_{M S I S, v}=35^{\circ}$ is the vertical beam width. The DVL consists of four narrow profiling beams, each with beam width angle $\varphi_{D V L}=2.2^{\circ} \ldots \ldots$

2-6 Robot Operating System (ROS) computation graph for the software components running the AUG's BSD computer, a Raspberry Pi. The red boxes are ROS nodes, the orange boxes are ROS topics, and the blue boxes are AUG hardware components. The figure highlights the connectivity between onboard sensor processes and AUG control processes. . . . . . . . . . . . .

2-7 Schematic diagram of an AUG conducting a sea-ice survey in the Arctic. The figure showcases how environment features make in-situ sea-ice survey dangerous for AUGs. The AUG is denied access to the ocean surface except for intermittent gaps between ice floes known as leads. Additionally, thick multi-year ice and pressure ridges serve as obstacles that the AUG must avoid when operating close to the sea surface. Figure adapted from [Jurohi, 2008].

3-1 Schematic diagram of AUG sampling sea-ice in the Arctic and transmitting compressed sea-ice information via satellite communication. The onboard sensor processing module reduces raw acoustic measurements into hierarchical sea-ice features: sea-ice presence, sea-ice thickness, and sea-ice roughness. Figure adapted from $[$ Lee et al., 2012] . . . . . . . . . . . . . . 
3-2 Schematic diagram of the MSIS sonar discerning thin sea-ice cover from free surface. $P_{R}, P_{T}$, and $P_{I S}$ represent the reflected, transmitted, and internal scattering acoustic waves, and together, they make up the components of acoustic scattering. The three subplots at the bottom of the figure show real acoustic scan-line data from laboratory testing that correspond with the bearings and surface conditions portrayed in the diagram (Note that the small initial peak in subplot A is caused by sidewall reflection in the test tank). 59

3-3 Examples of BSDF function $S$ for different surfaces. Note that only the reflected wave is portrayed in figure for simplicity. Surface A portrays a mirror-like surface that exhibits a planar wave reflection, Surface B portrays a glossy surface that exhibits a specular wave reflection, and Surface C portrays a Lambertian surface that exhibits uniform reflection in all directions. Figure adapted from $[$ VonHaarberg, 2018] . . . . . . . . . . . . .

3-4 Array of acoustic scan-lines for both ice and water surface conditions, for $5^{\circ}, 15^{\circ}$, and $25^{\circ}$ incidence angles. High intensity peaks that appear before and after the $0.4 \mathrm{~m}$ range are caused by sidewall and surface reflections that arise from the confined test tank environment that was used during data collection. Note that the data was collected in a freshwater environment. . .

3-5 Experiment setup for the laboratory tank testing with the MSIS in Woods Hole, MA. The figure shows the MSIS sonar mounted at a water depth of $42 \mathrm{~cm}$ and centered at the interface of free surface and ice cover. The ice was measured by hand to be $7.5 \mathrm{~cm}$ thick. . . . . . . . . . . . . . .

3-6 Percent Ice Cover (PIC) variation during laboratory tank testing in Woods Hole, MA. The left column of the figure shows the raw camera images of the various PIC conditions, while the right column of the figure shows the ice wedges highlighted in red for ease of interpretation. . . . . . . . . . . .

3-7 Field deployment in Saint-Fabien, QC. The deployment spanned four consecutive days, where the sampling location for each day is indicated by a gold star in the right subplot. The light-blue highlighted area shows the approximate portion of the bay that was covered with land-fast sea-ice for the duration of the deployment. Outside the land-fast sea-ice zone, marginal sea-ice cover was observed. . . . . . . . . . . . . . . . . 
3-8 Ice canoeing in Baie du Ha! Ha!, a small bay in Saint-Fabien, QC, situated on the Saint Lawrence River. Operated by a team of five people, the ice canoe is sledged over ice floes and pressure ridges, and paddled across sections of open water. Only a limited amount of scientific equipment may be deployed in the field because all equipment must be carried onboard the canoe. . . .

3-9 Preparation for data collection during field deployments in Saint-Fabien, QC. Sub-figure A shows a hole that was cut into the ice cover using an ice-saw so that under-ice MSIS sonar samples can be recorded. Sub-figure B shows the view from underneath the sea-ice cover, captured using a waterproof GoPro

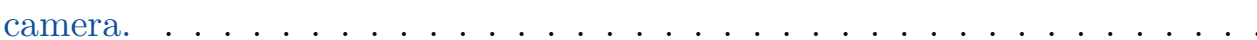

3-10 Percent Ice Cover (PIC) variation during field deployment testing in SaintFabien, QC. The left column of the figure shows the raw camera images of the various PIC conditions, while the right column of the figure shows the ice wedges highlighted in red for ease of interpretation. . . . . . . . . . .

3-11 Acoustic features of sea-ice for laboratory data collected in Woods Hole, MA. Peak-width, incidence angle, and maximum intensity are plotted as 3D coordinates in three-view format. The color of each point indicates percent ice cover (PIC), where dark blue denotes free-surface, yellow denotes full ice cover, and green or purple colors indicate partial ice cover. . . . . . . . . .

3-12 Acoustic features of sea-ice for field data collected in Saint-Fabien, QC. Peakwidth, incidence angle, and maximum intensity are plotted as 3D coordinates in three-view format. The color of each point indicates percent ice cover (PIC), where dark blue denotes free-surface, yellow denotes full ice cover, and green or purple colors indicate partial ice cover. . . . . . . . . . . . .

3-13 Receiver Operating Characteristic (ROC) curves for SSIM sea-ice detection, evaluated on laboratory and field datasets. Both laboratory and field datasets are evaluated with respect to single-ping and swath-of-pings classifiers. The optimal ROC curve points are indicated with orange stars, and the point of perfect classification and line of no-discrimination are included for reference. 
3-14 True Positive Rate (TPR) sea-ice detection performance as a function of Percent Ice Cover (PIC) for fixed levels of False Positive Rate (FPR). Both laboratory and field datasets are considered. High PIC conditions have better TPR performance than low PIC conditions, indicated by the general positive



4-1 Schematic diagram of the Velocity Shear Propagation (VSP) method being used to estimate water column currents. The VSP method uses forward propagation and back propagation to estimate ocean currents throughout the water column. . . . . . . . . . . . . . . .

4-2 Three factors of seafloor for the Kolumbo Volcano underwater crater: depth, slope, and orientation. Together, these three factors comprise the groundtruth map available to the AUG when performing navigation via the MFTAN algorithm. A kernel density plot is provided for each factor to convey the relative uniqueness of a particular location in the environment. . . . . .

4-3 Schematic diagram of seafloor factor extraction using the DVL instrument. To extract seafloor factors, a plane is fit to a set of bottom-contact points in 3D space. Then, seafloor depth, slope, and orientation can be readily extracted and used by the MF-TAN algorithm. . . . . . . . . . . . .

4-4 Offline ocean current sensing for a selection of AUG missions during the November 2019 deployment in the Southern Aegean Sea. Each row of the figure corresponds to a particular mission. The AUG starts each mission at the origin of the local mean coordinates (LMC) coordinate system. The decreased localization error provided by DVL-Odo compared to DR-DACC serves as evidence that the water column currents are being estimated accurately. . . . . . . . . . . . . . . . . . .

4-5 Bathymetric factor extraction for Dive A of the November 2019 deployment in the Southern Aegean Sea. The factor extraction shows how stability can be improved by considering successive DVL ensembles rather than just considering a single ensemble at each time-step. Note that the depth factors present at approximately $100 \mathrm{~m}$ water depth are caused by stratification in

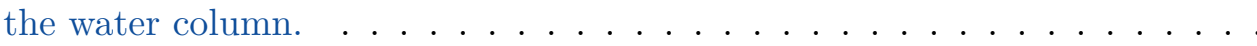


4-6 MF-TAN localization using three factors of the seafloor. The figure contains two subplots: Subplot A shows when the AUG performs localization within the Kolumbo crater, and Subplot B shows when the AUG performs localization outside of the crater. Each subplot highlights the three factor constraints in isolation, as well as the intersection of the constraints. When the factors are considered in combination with DVL-Odo position drift, the AUG is able to localize itself with respect to the ground-truth bathymetry.

4-7 Bathymetric factor extraction and MF-TAN navigation for Dive $\mathrm{F}$ of the November 2019 deployment in the Southern Aegean Sea. The depth, slope, and orientation factors correspond with the first, second, and third rows of the figure subplots. The MF-TAN method leverages three factors of seafloor bathymetry to significantly improve navigation performance over the DVLOdo and DR-DACC methods. . . . . . . . . . . . . . .

4-8 Multi-Factor Terrain-Aided Navigation (MF-TAN) for four dives during the November 2019 research deployment in the Southern Aegean Sea. MF-TAN is compared against DVL-Odo and DR-DACC, and both TAN fixes and GPS fixes are displayed. The MF-TAN navigation method consistently achieves the lowest navigation error compared to the other methods. . . . . . . . . .

5-1 Energy optimal propulsive speed as a function of hotel load and ocean current conditions for the 1D case. Color in both subplots indicates hotel load, where hotel load values vary between transit mode $(0.37 \mathrm{~W})$ and survey mode

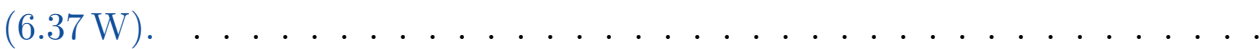

5-2 AUG range as a function of vehicle through-water speed and hotel load. This plot assumes an energy supply of $10 \mathrm{~kW} \mathrm{~h}$ and ignores the effect of ocean currents. The blue curve shows how the range-maximizing through-water speed of the AUG increases with increasing hotel load, echoing the result of equation $(5.3) \ldots \ldots \ldots \ldots \ldots \ldots \ldots$ 
5-3 AUG range as a function of vehicle through-water speed and ocean current conditions, shown for the transit hotel load in the left subplot and the survey hotel load in the right subplot. This plot assumes an energy supply of $10 \mathrm{kWh}$. The blue curve shows how the range-maximizing through-water speed of the AUG increases with decreasing favorable ocean currents, echoing the result of equation $(5.3) \ldots \ldots \ldots \ldots$

5-4 Average power draw of the buoyancy engine as a function of depth band size $z_{\text {dive }}-z_{\text {climb }}$ and AUG pitch angle $\phi$ assuming zero angle of attack and negligible vertical component of ocean currents. Since the average power grows exponentially for small depth bands and large pitch angles, exceeding $1000 \mathrm{~W}$ in some cases, the plot is capped at $24 \mathrm{~W}$ for easier interpretation. .

5-5 AVC given horizontal ocean current speeds $\leq 1 \mathrm{~ms}^{-1}$, where the vertical component is assumed to be negligible. In each subplot, the x-coordinate represents the cross-track component of the ocean current and the y-coordinate represents the co-linear component of the ocean current. The color shown in both subplots represents the optimal AUG through-water speed for transit and survey hotel loads given by the AVC control policy in equation (5.8). .

5-6 Transport cost improvement obtained by operating the DVL while transiting. By operating the DVL during transit, the AUG can perform AVC, which includes active steering to avoid cross-track and long-track drift caused by ocean currents. For ocean currents of small magnitudes, transport cost increases when the DVL is operated during transit. . . . . . . . . . .

5-7 AVC when applied to Dive A of the November 2019 deployment in the Southern Aegean Sea. The Hybrid Adaptive Speed curve shows the optimal AUG speed over time, which responds to varying ocean current conditions. The Hybrid Constant Speed is only optimized with respect to hotel load, so it does not change during the mission. The Forward Currents and Starboard Currents during mission are estimated via the VSP method. . . . . . . . . . 
5-8 EDBS as applied to Dive B of the November 2019 research deployment in the Southern Aegean Sea. The EDBS method is applied at heading intervals of $45^{\circ}$. For each AUG heading considered, the left subplot shows the transport cost as a function of depth band, where the yellow color indicates low transport cost and the blue color indicates high transport cost. Similarly, each right subplot shows the water column velocities at $1 \mathrm{~m}$ depth intervals with the resulting optimal depth band highlighted in orange. . . . . . . .

5-9 EDBS as applied to Dive F of the November 2019 research deployment in the Southern Aegean Sea. The EDBS method is applied at heading intervals of $45^{\circ}$. For each AUG heading considered, the left subplot shows the transport cost as a function of depth band, where the yellow color indicates low transport cost and the blue color indicates high transport cost. Similarly, each right subplot shows the water column velocities at $1 \mathrm{~m}$ depth intervals with the resulting optimal depth band highlighted in orange. . . . . . . . .

5-10 Energy-optimal through-water speed and over-ground speed for sea-ice survey using a reduced hotel load of $2.47 \mathrm{~W}$. The AUG over-ground speed is relatively constant across a fairly wide range of ocean current conditions, as indicated by the flat bowl shape in the 3D over-ground speed plot. Interestingly, the AVC control algorithm leads to a decrease in over-ground speed when small magnitude favorable ocean currents are encountered. . . . . . .

5-11 Overview of unattended AUG sea-ice survey mission scenario during the spring-summer sea-ice retreat. For this mission, the AUG departs Utqiagivik, Alaska, transits $450 \mathrm{~km}$ to the survey site on the Chukchi Plateau, performs a sea-ice survey, and then transits $450 \mathrm{~km}$ back to Utqiagivik. The feasibility of this mission depends on the resource management of the AUG. Figure adapted from bathymetric map by [Jakobsson et al., 2012]. . . . . . . . . . 130 


\section{List of Tables}

1.1 Requirements table for basin-scale Arctic observation technologies. Requirements are broken into categories of range, cost, and science. Technologies from remote sensing and in-situ sensing are considered, including aerial, sea surface, and subsea technologies. . . . . . . . . . . . . . . .

2.1 Review of hardware components including instantaneous power draw when the component is active, the expected duty cycle during a typical AUG mission, and the average power draw over the course of the mission. Hardware components fall into four main categories: vehicle propulsion and control systems, sensors, computers, and communication units. . . . . . . . . .

2.2 Comparison of battery chemistry configurations for the proposed AUG. Batteries are compared in terms of ambient energy capacity, derated energy capacity, whether or not they are rechargeable, and whether or not they are capable of smart querying. The mass and energy capacity figures listed in the table assume a standard number of AUG mid-sections corresponding with four battery pack sections. . . . . . . . . . . . . . . .

2.3 Review of notable parameters for the MSIS and DVL instruments. Note that the maximum and minimum range listed for the DVL instrument corresponds to DVL operating in bottom-track mode. When the DVL is operating in water-track mode, the instrument range envelope is tapered. . . . . . . . .

3.1 Density and speed of sound for three mediums: air, seawater, and seaice. Seawater is assumed to have a temperature of $-2{ }^{\circ} \mathrm{C}$ and a salinity of $32 \mathrm{~g} \mathrm{~kg}^{-1}$. Density and speed of sound values for sea-ice are taken from [Timco and Frederking, 1996] and [Vogt et al., 2008], respectively. . . . . . . 
3.2 Reflection and transmission coefficients for all acoustic transitions for the three mediums: air, seawater, and sea-ice. For this table, the incidence acoustic wave is assumed to be traveling normal to the boundary between the two mediums and that each medium is homogeneous. . . . . . . . . .

3.3 Review of MSIS actuation strategies in terms of instrument bearing and angular resolution for different sensing modes: sea-ice presence, sea-ice thickness, and sea-ice roughness. . . . . . . . . . . . . . . .

3.4 Percent Ice Cover (PIC) for two data-sets: a laboratory test data-set recorded during tank test operations in Woods Hole, MA, and a field deployment dataset recorder during ice-canoe operations in Saint-Fabien, QC. . . . . . . . .

3.5 True Positive Rate (TPR) and False Positive Rate (FPR) for two sea-ice detection modes: detection based on individual MSIS pings and detection based on an ensemble of neighboring MSIS pings. TPR and FPR rates are given for both laboratory and field experiments, which are described in Section 3.6. The metrics listed correspond with the optimal ROC curve points

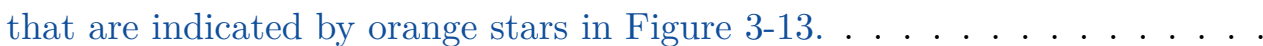

3.6 Analysis of how TPR performs on different PIC conditions, given three fixed values of FPR: $2 \%, 10 \%$, and $30 \%$. As expected, low PIC values are consistently more difficult to detect correctly compared to high PIC values. The number of points that fall into each category are given by the True Positive Count (TPC) and Condition Positive Count (CPC). Note that the TPR values shown in the table correspond with plotted points in Figure 3-14. . .

4.1 Comparison of Depth-Factor (DF) and Multi-Factor (MF) seafloor constraints during TAN fixes for the November 2019 deployment in the Southern Aegean Sea. DF and MF constraints are given as areas, corresponding to the portion of the ground-truth bathymetric map that is consistent with the factor(s) observed by the AUG. For each dive, median area $(\tilde{\sigma})$ and mean area $(\tilde{\sigma})$ are reported. The final column shows the percent reduction in area that occurs when using MF instead of DF. The final row of the table reports the aggregate of the five dives. . . . . . . . . . . . . 
4.2 Performance comparison between three methods of navigation: Dead Reckoning with Depth-averaged Current Correction (DR-DACC), DVL Odometry (DVL-Odo), and Multi-Factor Terrain-Aided Navigation (MF-TAN). Navigation error is given as a percentage of distance traveled for the three methods, and the median $(\tilde{\epsilon})$ and mean $(\bar{\epsilon})$ navigation errors in $[\mathrm{m}]$ are given for the MF-TAN method. Standard deviations are also listed for dives with multiple legs. The final row of the table shows the aggregate navigation performance across the five AUG missions. The AUG missions are from the November 2019 research deployment in the Southern Aegean Sea. . . . . . . . . . . . .

5.1 Review of hardware components that can be adaptively duty cycled during mission to reduce power expenditure. For resource-constrained AUG missions in the Arctic, operation of these instruments can be reduced to save energy while still meeting mission safety and science data collection requirements. .

5.2 Offline performance analysis between three AUG velocity control policies applied to November 2019 Southern Aegean Sea missions previously mentioned: constant velocity with the buoyancy engine alone, constant velocity with the hybrid buoyancy and thruster system, and AVC with the hybrid buoyancy and thruster system. The control methods are evaluated with respect to three metrics: mission time, average power draw, and transport cost. The most important metric for AUG energy efficiency, transport cost, shows that in this mission context the hybrid AUG performs approximately twice as well compared to the buoyancy engine alone, and the AVC method reliably outperforms the constant velocity controller. . . . . . . . . . . .

5.3 Performance comparison between the EDBS method and the default behavior of utilizing the full available water column (WC). Water column data is taken from two dives from the November 2019 deployment in the Southern Aegean Sea. The dives are vertically scaled to $80 \mathrm{~m}$ for ease of comparison. Dive B has significant velocity shear structure in the water column while Dive F is more uniform throughout the water column. Performance is evaluated at $45^{\circ}$ heading intervals, where performance metrics include transport cost and average speed over ground. . . . . . . . . . . . . . . 
5.4 Performance analysis of six AUG control policies applied to a hypothetical sea-ice survey at the Chukchi Plateau. The survey mission includes $\approx 900 \mathrm{~km}$ round-trip transit, with remaining energy used for science data collection. The baseline AUG control policy is a buoyancy driven propulsion using $26^{\circ}$ pitch. Each successive control policy demonstrates continual performance improvement, where performance improvement is given by the increase in total range relative to the baseline control policy. . . . . . . . . . . . . . . . 130 


\title{
Nomenclature
}

\author{
Acronyms \\ ACD Approximate Convex Decomposition \\ ADC Adaptive Duty Cycling \\ AUG Autonomous Underwater Glider \\ AUV Autonomous Underwater Vehicle \\ AVC Adaptive Velocity Control \\ BSD Backseat Driver computer \\ BSDF Bidirectional Scattering Distribution Function \\ CICE Community Ice CodE \\ DEM Digital Elevation Map \\ DOM Digital Orientation Map \\ DR Dead Reckoning \\ DR-DACC Dead Reckoning with Depth-averaged Current Correction \\ DSM Digital Slope Map \\ DVL Doppler Velocity Log \\ DVL-Odo Doppler Velocity Log Odometry \\ EDBS Exploitative Depth Band Selection
}




\begin{tabular}{|c|c|}
\hline EKF & Extended Kalman Filter \\
\hline FPR & False Positive Rate \\
\hline FSD & Floe Size Distribution \\
\hline FWHM & Full Width Half Maximum \\
\hline GPS & Global Positioning System \\
\hline GUI & Graphical User Interface \\
\hline IMU & Inertial Measurement Unit \\
\hline INS & Inertial Navigation System \\
\hline ITD & Ice Thickness Distribution \\
\hline JBPF & Jittered Bootstrap Particle Filter \\
\hline $\mathrm{KF}$ & Kalman Filter \\
\hline LBL & Long Baseline \\
\hline MF-TAN & Multi-Factor Terrain-Aided Navigation \\
\hline MIZ & Marginal Ice Zone \\
\hline MSIS & Mechanical Scanning Imaging Sonar \\
\hline $\mathrm{PF}$ & Particle Filter \\
\hline PIC & Percent Ice Cover \\
\hline $\mathrm{ROC}$ & Receiver Operating Characteristic \\
\hline ROS & Robot Operating System \\
\hline $\mathrm{ROV}$ & Remotely Operated Vehicle \\
\hline SIR & Sequential Importance Resampling \\
\hline SLAM & Simultaneous Localization and Mapping \\
\hline $\mathrm{SMC}$ & Sequential Monte Carlo \\
\hline
\end{tabular}




$\begin{array}{ll}\text { SSIM } & \text { Synoptic Sea-ece Mapping } \\ \text { TAN } & \text { Terrain-aided Navigation } \\ \text { TOF } & \text { Time of Flight } \\ \text { TPR } & \text { True Positive Rate } \\ \text { USBL } & \text { Ultra-Short Baseline } \\ \text { VSP } & \text { Velocity Shear Propagation } \\ \text { WC } & \text { Water Column }\end{array}$

\section{Notation}

AUG angle of attack

$\phi$

AUG pitch

$\boldsymbol{\psi} \quad$ AUG heading

$\theta$

AUG roll

AUG glide angle

$\delta \quad$ Relative alignment of ocean currents with AUG heading

Ocean current heading

Ocean current velocity vector 


\begin{tabular}{|c|c|c|}
\hline$v_{\text {buoy }}$ & Speed contribution from buoyancy engine (through-water speed) & {$\left[\mathrm{m} \mathrm{s}^{-1}\right]$} \\
\hline$v_{\text {prop }}$ & Speed contribution from propeller thruster (through-water speed) & {$\left[\mathrm{m} \mathrm{s}^{-1}\right]$} \\
\hline$E_{\text {pump }}$ & Energy cost of using ballast pump during bottom inflections & {$[\mathrm{J}]$} \\
\hline Pbuoy & Buoyancy engine power draw & {$[\mathrm{W}]$} \\
\hline$P_{\text {hotel }}$ & Hotel load power draw & {$[\mathrm{W}]$} \\
\hline$P_{\text {prop }}$ & Propulsive power draw & {$[\mathrm{W}]$} \\
\hline$P_{\text {total }}$ & Total instantaneous power draw & {$[\mathrm{W}]$} \\
\hline $\mathbf{u}$ & Input control vector & \\
\hline$\gamma$ & Percent of ballast volume pumped by buoyancy engine & {$[\%]$} \\
\hline$f_{T C}$ & Transport cost objective function & {$\left[\mathrm{J} \mathrm{m}^{-1}\right]$} \\
\hline$z_{\text {climb }}$ & Climb-to depth, component of AUG depth band & {$[\mathrm{m}]$} \\
\hline$z_{\text {dive }}$ & Dive-to depth, component of AUG depth band & {$[\mathrm{m}]$} \\
\hline$\varphi_{D V L}$ & Acoustic beam width of the DVL & \\
\hline$\varphi_{M S I S}$ & Acoustic beam width of the MSIS & \\
\hline$\dot{\boldsymbol{\psi}}_{M S I S}$ & Angular resolution setting of MSIS & {$\left[{ }^{\circ} \mathrm{s}^{-1}\right]$} \\
\hline$\psi_{M S I S}$ & Bearing setting of MSIS ( $0^{\circ}$ is vertically upwards) & \\
\hline$k_{M S I S}$ & Gain setting of MSIS & {$[\%]$} \\
\hline$r_{M S I S}$ & Range setting of MSIS & {$[\mathrm{m}]$} \\
\hline$\rho$ & Density of medium & {$\left[\mathrm{kg} \mathrm{m}^{-3}\right]$} \\
\hline$c$ & Speed of sound in medium & {$\left[\mathrm{m} \mathrm{s}^{-1}\right]$} \\
\hline$R$ & Reflection coefficient & \\
\hline$T$ & Transmission coefficient & \\
\hline$S\left(\omega_{i}, \omega_{r}\right)$ & $\mathrm{BSDF}$ & \\
\hline
\end{tabular}


$H \quad$ Transmission loss

$L_{p} \quad$ Sound pressure level

$L_{s} \quad$ Source level

$T_{s} \quad$ Target strength

$\epsilon$

Navigation error

$[\mathrm{m}]$

$\sigma$

Extent of state space for TAN fix

$\left[\mathrm{m}^{2}\right]$

$\mathbf{x}_{t}$

State at time $t$

$\mathbf{y}_{t} \quad$ Observation at time $t$

$\mathbf{Y}_{t} \quad$ Observation history up through time $t$ 
This page intentionally left blank. 


\section{Chapter 1}

\section{Introduction}

\subsection{Motivation for Studying Arctic Sea-ice}

The Arctic is the most rapidly warming region on Earth and over the past several decades these rising temperatures have had a substantial impact on the region's seasonal sea-ice cover and volume [Serreze and Barry, 2011, Stammerjohn et al., 2012]. Changing conditions in the Arctic have broad ramifications for the global Earth system, including rising global temperatures, as well as biological, chemical, physical, and societal impacts [Francis and Skific, 2015, Cohen et al., 2018, Arrigo et al., 2014, Barnhart et al., 2014, Jakobsson et al., 2014, Christiansen et al., 2014]. Diminished sea-ice volume results in diminished latent heat thermal buffering capacity, which accelerates warming of the Earth's oceans [Kwok and Cunningham, 2015, Jeffries et al., 2013, Jackson et al., 2012]. Thus it is critical to accurately understand Arctic sea-ice inventory. In addition to rising temperatures, positive feedback mechanisms of albedo and momentum transfer are causing the decline of Arctic sea-ice to accelerate. Sea-ice, and particularly the snow settling on top of sea-ice, has a characteristic albedo that is among the highest of all natural materials found on Earth's surface, causing sea-ice and snow to reflect the majority of incoming solar radiation back into the atmosphere. When sea-ice melts and exposes the ocean below, the low-albedo seawater absorbs the majority of the solar radiation causing the sea-surface temperature to rise, which provokes more sea-ice melting [Nicolaus et al., 2012]. Additionally, as seaice cover declines, the ice pack is less able to dampen sea surface kinetics, amplifying the mechanical break-up of sea-ice, which leads to further lateral melting [Zhang et al., 2015]. To better understand the global sea-ice latent heat budget and the processes that govern 
sea-ice dynamics, it is essential to improve our understanding of Arctic sea-ice volume as well as the transfer of heat and momentum at the sea-ice boundary. This requires overcoming the intrinsic challenges of sea-ice survey operations.

The Arctic Ocean has remained as one of the most forbidding environments for humans since prehistoric people first ventured out onto the ice floes of Eurasia. Historical records recount numerous expeditions during $19^{\text {th }}$ and $20^{\text {th }}$ centuries that met tragic ends, such as the Franklin expedition of 1845 [Beattie and Geiger, 2017], Greely expedition of 1882 [Todd, 2017], and Amundsen's 1928 failed rescue attempt of the Italia expedition [Hovdenak, 1935]. Sea-ice cover in the Arctic Ocean is such a barrier for humans that the North Pole was indisputably reached only after humans reached the South pole; the first ship to reach the North Pole was a nuclear powered submarine on its second attempt [Griffin, 2013], less than 10 years before humans landed on the moon. Despite the advance of modern technologies, the Arctic Ocean's challenges and dangers persist as barriers for scientific observation, imposing significant limitations on observing systems. Major operational constraints include the fact that relevant areas of sea-ice study are spatially remote, often many hundreds of kilometers away from nearest land contact or operating base. In terms of physical dangers, weather, wildlife, and sea-ice are persistent threats to observational hardware. For example, pressure ridges formed from the collision of ice floes can exceed $30 \mathrm{~m}$ in draft height, sometimes impinging upon the seafloor, making under-ice technologies particularly vulnerable.

\subsection{Review of Arctic Sea-ice Survey Technologies}

Conventional Arctic sea-ice observing systems include remote aerial and satellite systems, as well as in-situ sea surface and subsea systems. Remote aerial and satellite observation is able to acquire surface data with the greatest spatial extent while maintaining kilometerto-meter spatial resolution and day-to-week temporal resolution [Markus et al., 2017, Kwok and Cunningham, 2015, Ardhuin et al., 2018]. These systems are particularly well suited for measuring sea-ice area coverage and determining the sea-ice boundary. Satellite observation is, however, generally limited by the difficulty in accurately determining sea-ice thickness and volume [Schweiger et al., 2011], as well as the inability to assess physical characteristics such as heat and momentum transfer. Ice-hardened vessels can be used to observe these physical characteristics, but they are costly to operate and environmentally 
intrusive because the transit path to the study site causes disturbance to the sea-ice being studied [Lee et al., 2012]. Although other in-situ surface technologies such as moorings and ice-tethered buoys [Richter-Menge et al., 2006, Toole et al., 2011] are minimally intrusive and able to operate unattended, they are often crushed between ice floes [Jackson, 2016] and only provide point measurements.

Subsea observational platforms, including remotely operated vehicles (ROVs) [Nicolaus et al., 2012] and tow bodies, require the support of surface vessels for operation, but have limited spatial extent and are infeasible for scaling up to basin-scale observation at meaningful spatial and temporal resolution. Although human occupied submersibles have operated under ice cover in the Arctic [Sagalevitch, 2013], conventional electric and diesel-electric submarines are not considered viable for under-ice research because of their limited endurance and the associated risk to the crew. In contrast, nuclear-powered submarines are perhaps the most-capable of all platforms for in-situ Arctic sea-ice observation because these vessels have access to an effectively limitless energy supply while underway, producing water, oxygen, and heat for crew, as well as ample electricity for instrumentation. Important scientific insights into Arctic sea-ice processes have relied on nuclear-powered military submarines [Wadhams and Horne, 1980]. The obvious downside to using nuclear submarines for Arctic research is that they are extremely costly to operate and are the exclusive domain of only a few of the world's militaries. More recently, autonomous underwater vehicles (AUVs), including autonomous underwater gliders (AUGs), have demonstrated the ability to operate under ice [Kukulya et al., 2016, Kunz et al., 2009, Williams et al., 2015]. With notable exceptions [Kaminski et al., 2010, Furlong et al., 2012, Boeing, 2017], most AUVs do not possess the required range to conduct basin-scale Arctic sea-ice surveys. AUGs, however, routinely operate in polar waters [Lee et al., 2017, Zhou et al., 2019, Miles et al., 2016], have successfully completed multiple trans-Atlantic missions [Ramos et al., 2018, Willis, 2009] and with appropriate modification, may be viable for basin-scale Arctic operations. Scaling up to persistent basin-scale synoptic observation using underwater platforms will require miserly power budgets coupled with low unit costs.

A review of Arctic observation system technologies is provided in Table 1.1, which highlights whether or not an observational platform meets the requirements for basin-scale in-situ Arctic study. The table considers three aspects of Arctic observing capabilities: range, cost, and analytical sensing. Range performance is evaluated with respect to the 
ability to traverse more than $5000 \mathrm{~km}$. This range requirement is derived from the remote aspect of the Arctic region. Costs are evaluated with respect to capital cost and daily operating cost and are reported as orders of magnitude estimates which are not meant to convey precision, but rather semi-quantitative relationships between survey methods. Analytical sensing capabilities are evaluated with respect to the ability to measure sea-ice volume, sea-ice area, heat transfer at the sea-ice interface, momentum transfer at the seaice interface, and whether or not a given technology is environmentally intrusive. In this context, intrusive signifies that the observing technology creates a significant disturbance to sea-ice in the area being studied.

\begin{tabular}{|c|c|c|c|c|c|c|c|c|}
\cline { 2 - 8 } \multicolumn{1}{c|}{} & $\begin{array}{c}\text { Traverse } \\
>5000 \mathrm{~km}\end{array}$ & $\begin{array}{c}\text { Capital } \\
\text { Cost }\end{array}$ & $\begin{array}{c}\text { Operating } \\
\text { Cost (Daily) }\end{array}$ & $\begin{array}{c}\text { Sea-ice } \\
\text { Volume }\end{array}$ & $\begin{array}{c}\text { Sea-ice } \\
\text { Area }\end{array}$ & $\begin{array}{c}\text { Heat } \\
\text { Transfer }\end{array}$ & $\begin{array}{c}\text { Momentum } \\
\text { Transfer }\end{array}$ & Nonintrusive \\
\hline Satellites & $\checkmark$ & $10^{8}$ & $10^{4}$ & - & $\checkmark$ & $x$ & $x$ & $\checkmark$ \\
\hline Airplanes & $\checkmark$ & $10^{5}-10^{8}$ & $10^{4}$ & - & $\checkmark$ & $x$ & $x$ & $\checkmark$ \\
\hline $\begin{array}{c}\text { Icebreaker } \\
\text { Campaigns }\end{array}$ & $\checkmark$ & $10^{8}-10^{9}$ & $10^{5}$ & $\checkmark$ & $\checkmark$ & $\checkmark$ & $\checkmark$ & $x$ \\
\hline $\begin{array}{c}\text { Ice Camps } \\
\text { Ice-Tethered } \\
\text { Buoys }\end{array}$ & $\times$ & $10^{8}-10^{9}$ & $10^{2}-10^{5}$ & $\checkmark$ & $\checkmark$ & $\checkmark$ & $\checkmark$ & - \\
\hline $\begin{array}{c}\text { Under-Ice } \\
\text { Moorings }\end{array}$ & $\times$ & $10^{6}$ & $10^{2}$ & $\checkmark$ & $\checkmark$ & $\checkmark$ & $\checkmark$ & $x$ \\
\hline $\begin{array}{c}\text { Nuclear } \\
\text { Submarines }\end{array}$ & $\checkmark$ & $10^{6}$ & $10^{0}$ & $\checkmark$ & $\checkmark$ & $\checkmark$ & $\checkmark$ & $\checkmark$ \\
\hline ROVs & $x$ & $10^{4}-10^{7}$ & $10^{5}$ & $\checkmark$ & $\checkmark$ & $\checkmark$ & $\checkmark$ & $\checkmark$ \\
\hline AUVs & $x$ & $10^{5}-10^{7}$ & $10^{5}$ & $\checkmark$ & $\checkmark$ & $\checkmark$ & $\checkmark$ & $x$ \\
\hline AUGs & - & $10^{5}$ & $10^{3}$ & $\checkmark$ & $\checkmark$ & $\checkmark$ & $\checkmark$ & $\checkmark$ \\
\hline
\end{tabular}

Table 1.1: Requirements table for basin-scale Arctic observation technologies. Requirements are broken into categories of range, cost, and science. Technologies from remote sensing and in-situ sensing are considered, including aerial, sea surface, and subsea technologies.

As Table 1.1 illustrates, the general drawback of ROVs and AUVs is limited range capability for basin-scale Arctic operations. Although AUVs can overcome range limitations by scaling up in size, this leads to an increasing trend in capital and operating costs. This leaves AUGs as the Arctic observation technology that offers one of the most promising compromises between range, cost, and observational capability.

Figure 1-1 reviews the design space of subsea Arctic-sampling technologies in terms of mass and range, including four main size classes: extra large vehicles (Echo Voyager [Boeing, 2017], Theseus [Butler and den Hertog, 1993]), large vehicles (Sentry [Kaiser et al., 2016], Bluefin21 [Lehmenhecker and Wulff, 2013], Autosub [Furlong et al., 2012]), medium vehicles (Tethys [Bellingham et al., 2010], Slocum [Jones et al., 2014], Spray [Sherman et al., 2001], Seaglider [Lee et al., 2017]), and small vehicles (Remus [Kukulya et al., 2010]). The highlighted area of the design space is comprised of vehicles with sufficient range and science 
capabilities to pursue meaningful deployments in the Arctic, while requiring only modest costs. Like most marine vessels, unit costs of robotic underwater vehicles tend to correlate with displacement, and operating costs are influenced substantially by the infrastructure requirements for launch and recovery.

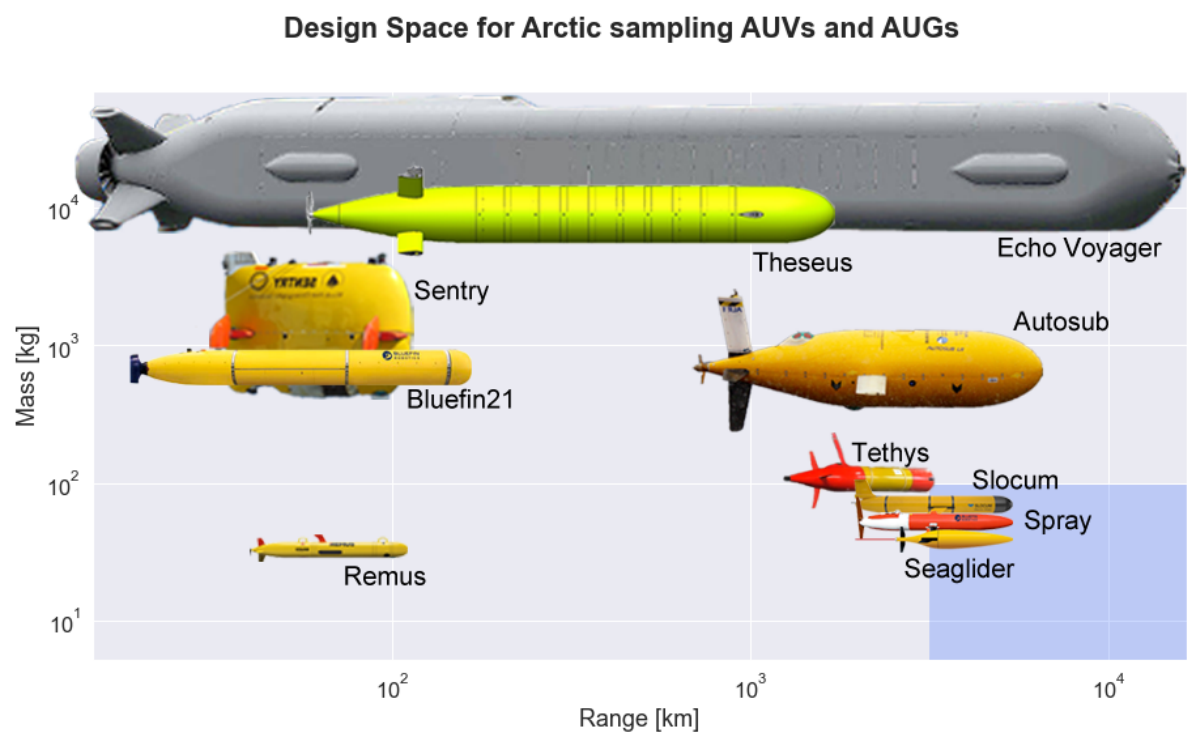

Figure 1-1: Overview of design space for under-ice surveying AUVs and AUGs in terms of mass and range, where the blue highlighted region indicates the area of interest within the design space. The top-right corner of each vehicle image indicates its approximate numerical value within the design space. The scaling of the vehicle images is approximate.

The complexity of Arctic observation suggests that optimal survey methods would make use of a blend of several technologies: satellites providing synoptic basin-scale estimate of sea-ice extent, periodic icebreaker campaigns to collect physical samples within small regions of interest, and persistent long-endurance AUGs and AUVs to record in-situ observations across large under-ice transects. In such an observing network, AUGs would be limited by energy storage constraints which directly influence range and science capabilities. Therefore, this thesis proposes a hybrid AUG architecture that includes a high-efficiency thruster, low-power acoustic sensors, and energy-optimal control policies for unattended transit and survey. Efficient utilization of onboard resources is necessary for AUGs and AUVs to be effective for sea-ice observing networks. 


\subsection{Need for Static and Dynamic Sea-ice Inventory}

Long-range AUGs have the potential to supplement the observational capability of both static and dynamic sea-ice quantities. Static quantities refer to total inventory at a moment in time, whereas dynamic quantities refer to the change in inventory over a time duration. For example, for Arctic sea-ice survey operations, it is valuable to measure both the static inventory and dynamic inventory of sea-ice variables. In particular, understanding static and dynamic inventory sea-ice volume in the Arctic is of critical importance for understanding Earth's global heat budget [Jackson et al., 2012, Wadhams, 2012, Maksym, 2019].

While aerial observation systems face difficulties in measuring sea-ice volume [Schweiger et al., 2011], an in-situ sea-ice observing AUG can directly measure sea-ice volume using a principle known to humans for millennia: Archimedes' Principle, originally stated by Archimedes circa 250B.C. in 'On Floating Bodies' [Heath et al., 2002]. Archimedes' Prin-

ciple states that a body submersed in fluid experiences an upward buoyant force that is equal in magnitude to the weight of fluid being displaced. Thus, an AUG with knowledge of sea-ice and sea-water densities can measure vertical sea-ice displacement using onboard acoustic sensing, allowing the AUG to directly measure sea-ice volume. Therefore, with persistent AUG sea-ice operations in the Arctic, it is possible to estimate static and dynamic inventory of sea-ice volume.

\subsection{Navigating the New Arctic}

Aside from their ability to supplement crucially important sea-ice data products, AUGs are prepared to adapt to evolving environmental conditions in the Arctic. As sea-ice cover and sea-ice volume decline, ocean circulation patterns are changing [Kwok et al., 2013, Horvat et al., 2016, Meneghello et al., 2018], Arctic freshwater content is increasing [Haine et al., 2015, Carmack et al., 2016, Lique et al., 2016], sea-surface waves and turbulence are intensifying [Thomson and Rogers, 2014, Zhang et al., 2013, Asplin et al., 2012], and annual heat fluxes are rising [Screen and Simmonds, 2010]. Evolving environmental conditions in the Arctic put a premium on capable and adaptable sea-ice observing technology.

The upcoming chapters of this thesis review an architecture for a modified AUG with specialized hardware and software components that allow the vehicle to collect valuable sea-ice data while adapting to dynamic and dangerous environmental conditions. 


\subsection{Thesis Contributions}

This thesis aims to extend the autonomous capabilities of AUGs to enable basin-scale in-situ characterization of sea-ice in the Arctic. To support this central aim, this thesis includes three primary contributions: a hierarchical acoustics-based sea-ice characterization scheme to perform science data collection and assess environment risk, a multi-factor terrain-aided navigation method that leverages bathymetric features and active ocean current sensing to limit localization error, and a set of energy-optimal propulsive and hotel policies that react to evolving environmental conditions to improve AUG endurance.

\subsection{Thesis Organization}

- Chapter 1 provides motivation for studying Arctic sea-ice and outlines viable technologies for studying Arctic sea-ice.

- Chapter 2 describes an architecture for an AUG that is capable of providing persistent basin-scale synoptic observation of Arctic sea-ice.

- Chapter 3 proposes an approach for onboard characterization of sea-ice using a mechanical scanning imaging sonar.

- Chapter 4 proposes a method for terrain-aided navigation that leverages multiple seafloor features and real-time ocean current estimation.

- Chapter 5 describes a set of energy optimal control policies that can be used to improve AUG endurance.

- Chapter 6 provides a conclusion of the thesis and offers recommendations for future work. 
This page intentionally left blank. 


\section{Chapter 2}

\section{Proposed Architecture for}

\section{Autonomous Underwater Glider}

\section{$2.1 \quad$ Introduction}

AUVs enhance the scope of Arctic sea-ice observation networks due to their ability to make in-situ observations of sea-ice volume, sea-ice area, heat-transfer at the sea-ice interface, and momentum-transfer at the sea-ice interface. Within the ecosystem of viable sea-ice observing AUVs, there is a particular subset of design space that is ideal for enabling persistent basin-scale synoptic observation of Arctic sea-ice: modest cost vehicles that are capable of long-range missions. In particular, three commercially available AUGs shown in Figure 1-1 coincide with this subset of design space: the Slocum AUG, the Spray AUG, and the Seaglider AUG.

This thesis builds upon the standard Slocum G3 electric AUG configuration by proposing a hardware and software architecture that enables the AUG to conduct long-range sea-ice survey missions in the Arctic. The modified Slocum AUG is named: Polarsentinel.

The proposed architecture is derived from four fundamental requirements: scientific data collection, long-range travel, unattended operation, and survival. Scientific data collection is at the heart of why Arctic sea-ice survey is conducted in the first place: to better understand processes governing sea-ice and related climate systems. Therefore, the AUG must be capable of making observations of sea-ice and related oceanographic variables such as temperature and momentum flux. Long-range travel is essential to enable synoptic sea- 


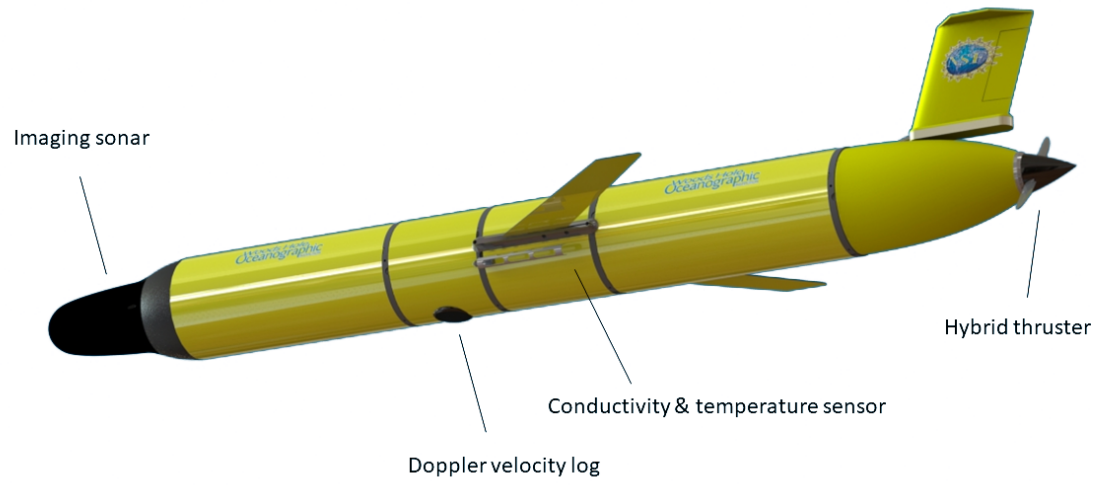

Figure 2-1: Rendering of Polarsentinel, the proposed AUG architecture. Polarsentinel is a modified Slocum G3 Electric Glider. Physical modifications include: MSIS for sea-ice characterization, DVL for environment state estimation and navigation, hybrid thruster design for increased efficiency, and updated battery pack for increased energy capacity.

ice observation with only a limited number of vehicles. Additionally, long-range travel is necessary to support launch and recovery maneuvers in remote areas of the world where rendezvous points may be far away from the survey location. Therefore, the the AUG must be capable of long-range travel on the order of basin-scale missions. Unattended operation is required to keep costs of Arctic sea-ice survey from growing exponentially with the amount of infrastructure needed to enable operation. Therefore, the AUG must be capable of unattended operation such that the AUG is not dependent on persistent surface vessel support or acoustic transponders preemptively placed in the environment. Finally, AUG survivability is necessary for recovering the valuable scientific data that was collected in satisfaction of the first requirement. Additionally, AUG survivability is necessary for persistent observation of Arctic sea-ice: similar to SpaceX's motivation for developing reusable rockets, it is difficult to perform persistent operations with single-use technologies. Therefore, the AUG must be expected to survive Arctic sea-ice survey operations, which entails the ability to accurately sense environment state and adaptively adjust the mission plan in response to apparent risks. Together, these four requirements serve as the guiding principles in the derivation of the proposed AUG architecture. Notably, each requirement is connected to a central theme: intelligent allocation of AUG resources. 


\subsection{Review of Hardware Components}

A hardware architecture is proposed that utilizes a prototype high-efficiency hybrid thruster and commercially available low-power acoustic sensors that enable real-time environmental state estimation. To further improve energy efficiency, the thesis considers low-level optimization behaviors for hybrid propulsion and acoustic sensing in conjunction with onboard data interpretation that allows for efficient information transfer during vehicle surfacing.

AUG power expenditure can be divided into two primary categories: propulsive and hotel. As a hybrid vehicle, propulsive power includes power draw for the buoyancy engine and power draw to drive the propeller. Hotel electric power, or hotel load, consists of all non-propulsive systems running onboard the AUG, including computers and active control systems, sensors, and communication equipment. Sensors running onboard the vehicle include a Mechanical Scanning Imaging Sonar (MSIS) for sea-ice and sea-state characterization, a Doppler Velocity Log (DVL) for navigation and ocean current estimation, a Conductivity Temperature and Depth (CTD) sensor for measuring proprioceptive water-column variables, and an Inertial Measurement Unit (IMU) for measuring vehicle accelerations. A summary of the AUG's hardware components and corresponding power requirements are given in Table 2.1.

For normal flight conditions, not all of the components mentioned in Table 2.1 are necessary. For example, the communication components are only used when the AUG is at the surface, which is a small fraction of total mission time. By toggling different components on and off, a set of possible operating conditions can be defined. Two operating modes that are commonly defined for underwater vehicles conducting science missions are transit mode and survey mode. When the AUG is in transmit mode, scientific and nonessential systems are turned off to limit power expenditure while traveling to science targets. Conversely, when the AUG is in survey mode, analytical sensors are turned on to enable observation of the science target. Hotel configuration can, however, be expressed as gradations between transit and survey mode, instead of just binary states. For example, in instances of moderate ocean currents, it may be beneficial to operate the DVL while transiting to improve AUG navigation via active steering to counteract cross-track ocean currents even though the hotel load is increased. Further treatment of hotel load optimization is discussed in Chapter 5. Transit mode and survey mode are defined as the upper and lower bound of the AUG's 


\begin{tabular}{|l|c|c|c|}
\hline \multicolumn{1}{|c|}{$\begin{array}{c}\text { Hardware } \\
\text { Component }\end{array}$} & $\begin{array}{c}\text { Instantaneous } \\
\text { Power Draw } \\
{[\mathrm{W}]}\end{array}$ & $\begin{array}{c}\text { Duty } \\
\text { Cycle } \\
{[\%]}\end{array}$ & $\begin{array}{c}\text { Average } \\
\text { Power Draw } \\
{[\mathrm{W}]}\end{array}$ \\
\hline Hybrid Thruster (min) & 1.09 & 98.4 & 1.07 \\
\hline Hybrid Thruster (max) & 18.10 & 98.4 & 17.80 \\
\hline Buoyancy Engine & 101.00 & 1.6 & 1.60 \\
\hline Altimeter & 0.41 & 41.2 & 0.17 \\
\hline Pitch Controller & 2.18 & 0.9 & 0.02 \\
\hline Rudder & 2.44 & 1.0 & 0.02 \\
\hline MSIS (700 kHz) & 3.00 & 100.0 & 3.00 \\
\hline DVL (600 kHz) & 2.00 & 100.0 & 2.00 \\
\hline CTD & 0.14 & 100.0 & 0.14 \\
\hline Micro IMU & 0.40 & 100.0 & 0.40 \\
\hline Flight Computer & 0.16 & 100.0 & 0.16 \\
\hline BSD & 0.40 & 100.0 & 0.40 \\
\hline Radio Modem (900 MHz) & 21.00 & 0.00 & 0.00 \\
\hline Satellite Communication & 6.00 & 1.00 & 0.06 \\
\hline
\end{tabular}

Table 2.1: Review of hardware components including instantaneous power draw when the component is active, the expected duty cycle during a typical AUG mission, and the average power draw over the course of the mission. Hardware components fall into four main categories: vehicle propulsion and control systems, sensors, computers, and communication units.

hotel load, without including the power draw from communication equipment. This yields an average power demand for transit and survey mode of $0.37 \mathrm{~W}$ and $6.37 \mathrm{~W}$ respectively.

\subsection{Hybrid Propulsion}

The hybrid AUG is unique among robotic underwater vehicles in that it is able to utilize buoyancy engine propulsion and/or propeller thruster propulsion, while reaping the benefits of both propulsive strategies: efficiency at low speeds via the buoyancy engine and variable freedom of speed variability via the propeller thruster. The hybrid thruster is particularly useful because it enables the AUG to operate despite adverse or cross-track currents, whereas an AUG equipped with buoyancy engine alone would be susceptible to being swept off-course. Prior work suggests a strategy wherein AUGs utilize high-speed transport to mitigate the negative impact of adverse or cross-track currents [Jenkins et al., 2003]. The ability to travel at higher speeds is especially useful in the context of sea-ice surveys because higher speed may allow the AUG to evade dynamic sea-ice cover in order to reach a safe 
surfacing location.

In addition to the wider velocity envelope, hybrid thrust improves flexibility for AUG path planning by not requiring that the AUG rely entirely on the sawtooth path associated with the buoyancy engine. The AUG can instead travel at an arbitrary depth band without loss of speed or efficiency, which may be beneficial for scientific observation, navigation and localization, obstacle avoidance, or improved efficiency in shallow waters. Using the buoyancy engine in conjunction with the thruster avoids a drawback common to AUVs of expending energy to achieve neutral stability. AUVs often must contend with this because they are ballasted slightly positively buoyant as a safety precaution [Jenkins et al., 2003], [Bellingham et al., 1995]. Since the AUG does not have access to elevator control, it can be challenging to stabilize with thruster and pitch controller alone [Claus et al., 2012]. By providing a stabilizing force via buoyant loading of the glide surfaces, the buoyancy engine boosts the efficiency of the thruster. Finally, this flexibility of two parallel propulsive strategies makes the AUG more redundant to catastrophic failure during the mission, and thus, single-fault tolerant with respect to propulsion.

To formalize the discussion of energy efficiency, the transport cost function $f_{T C}$ is defined to be equal to the total energy expended per distance traveled, $f_{T C}=\frac{\Delta E}{\Delta x}$. Thus, lower values of transport cost indicated greater energy efficiency of AUG travel.

\subsubsection{Buoyancy Engine}

The buoyancy engine allows the AUG to travel efficiently through the water column while generating forward motion in a sawtooth trajectory, with the AUG inflecting downward once it reaches the top of its flight band and inflecting upward upon reaching the bottom of its flight band. Through the use of ambient hydrostatic pressure, the AUG only needs to actively pump ballast at the lower inflection points, enabling low energy expenditure during the majority of the trajectory. The buoyancy engine's efficiency decreases, however, in proportion to a narrowing flight band because a narrowing flight band corresponds with an increase in the frequency of AUG inflections. Thus, efficiency is highest when the flight band includes the full depth range of the AUG. Analytical models adapted from Jenkins et al. [Jenkins et al., 2003] suggest that the transport cost of buoyancy engine travel is minimized at approximately $12^{\circ}$ pitch angle, although steeper angles yield greater horizontal speeds. AUG designs such as the Slocum and Seagliders also utilize isopycnal hulls with 
compressibility characteristics roughly matching seawater, further minimizing buoyancy engine energy expenditure [Webb, 2006].

\subsubsection{Propeller Thruster}

While the buoyancy engine is efficient for travel at relatively low speeds, large depth bands, and shallow pitch angles, the hybrid thruster is better suited for missions requiring higher speeds or narrow flight bands. An expanded vehicle dynamics envelope provides more flexibility for mission planning and adaptive control to contend with adverse environmental conditions often encountered in the Arctic. Large portions of the Arctic basin are on continental shelves and have shallow depths. For example, much of the Chukchi Sea is between $25 \mathrm{~m}$ and $50 \mathrm{~m}$ deep. When including an appropriate safety margin for possible sea-ice cover and inflection at depth, the resulting flight band is narrow and energetically expensive for buoyancy engine transit. Although AUGs are generally designed to travel throughout their entire depth range during transit, there are control methods that can be used for hybrid AUGs to travel at constant depths. Claus and Bachmeyer demonstrate the ability to use a linear reduced order model of AUG dynamics paired with a linear quadratic regulator controller to minimize energy loss due to lift induced drag from excess buoyant force [Claus and Bachmayer, 2016].

Optimization of AUG thrust for energy conservation requires understanding of the relationship between through-water speed and thruster input power. This can be described as an efficiency curve, which is given below. The propulsive power is specified in $\mathrm{W}$ and the through-water speed of the AUG $v_{t w}$ is given in $\mathrm{ms}^{-1}$.

$$
P_{\text {prop }}\left(v_{t w}\right)=3.79 v_{t w}^{3}+1.99 v_{t w}^{2}-0.22 v_{t w}+1.09
$$

This theoretical model for a prototype thruster currently under development considers propeller, motor, and motor controller losses, and is designed to provide a wide velocity envelope while maintaining efficiency across its range.

Figure 2-2 shows the comparison between buoyancy engine efficiency and prototype thruster efficiency when the AUG is operating in transit mode. Note that the two propulsive modes have different dependencies for efficiency: the buoyancy engine depends on pitch angle and depth band, while the thruster depends on through-water speed. The thruster is 




Figure 2-2: Comparison of transport cost between the buoyancy engine and the prototype thruster when operating in transit mode. The blue solid curves correspond to the transport costs of the buoyancy engine, while the yellow, orange, and red dashed lines correspond with the prototype thruster.

most efficient at $0.5 \mathrm{~m} \mathrm{~s}^{-1}$, independent of depth band and pitch angle. This figure suggests that a fairly broad thruster velocity envelope is more efficient than the buoyancy engine when operating within a narrow depth band. However, these efficiency curves do not take into account the effect of science payload or ocean currents. For increased science payload or adverse ocean current magnitudes, it becomes more efficient to travel at higher speeds.

\subsection{Battery Pack Design}

A Slocum AUG can be equipped with an extended endurance pack configuration comprised of lithium primary cells, providing approximately $10 \mathrm{~kW} \mathrm{~h}$ at ambient Arctic ocean temperatures. Although Lithium primary batteries are not rechargeable, they allow for a three-fold increase in the AUG's onboard energy capacity compared to lithium ion secondary batteries, to help meet the range requirements of an Arctic mission. Both classes of lithium battery chemistry have thermal characteristics that are superior to most other battery chemistries, making them better suited for Arctic missions where water temperature is approximately $-2{ }^{\circ} \mathrm{C}$. Although the extended endurance pack configuration is necessary to meet the range requirements of Arctic survey, the lithium secondary pack configuration has been instru- 


\begin{tabular}{|l|c|c|c|c|c|}
\hline Battery Chemistry & $\begin{array}{c}\text { Ambient } \\
\text { Capacity } \\
{[\mathrm{kW} \mathrm{h}]}\end{array}$ & $\begin{array}{c}\text { Derated } \\
\text { Capacity } \\
{[\mathrm{kW} \mathrm{h}]}\end{array}$ & $\begin{array}{c}\text { Derating } \\
\text { Factor } \\
{[\%]}\end{array}$ & Recharge & $\begin{array}{c}\text { Smart } \\
\text { Query }\end{array}$ \\
\hline Alkaline Primary & 2.2 & 1.6 & 72 & $\boldsymbol{X}$ & $\boldsymbol{X}$ \\
\hline Lithium Secondary & 3.2 & 2.1 & 65 & $\checkmark$ & $\checkmark$ \\
\hline Lithium Primary & 12.0 & 10.0 & 83 & $\mathbf{X}$ & $\mathbf{X}$ \\
\hline
\end{tabular}

Table 2.2: Comparison of battery chemistry configurations for the proposed AUG. Batteries are compared in terms of ambient energy capacity, derated energy capacity, whether or not they are rechargeable, and whether or not they are capable of smart querying. The mass and energy capacity figures listed in the table assume a standard number of AUG mid-sections corresponding with four battery pack sections.

mental in the development of the proposed architecture because of its rechargeable and smart-querying features.

In the proposed architecture, four distinct battery pack sections are considered: the pitch pack, the payload pack, the aft-short pack, and the aft-long pack. The four packs with lithium secondary pack configuration are shown in Figure 2-3. Each battery pack chassis section has different design considerations, where the chassis sections are designed via Computer-Aided Design (CAD) and constructed using 3D printers. 3D printing allows for a high degree of customization and an ability to limit weight without compromising strength by utilizing internal lattice structure during construction.

The pitch pack has two functions: contributing to the energy supply of the AUG and providing a pitch moment by sliding forward and aft. The position of the pitch pack along the axis of the AUG body helps establish the glide angle $\xi$ for efficient travel through the water column in coordination with the buoyancy engine. As a result, the pitch pack chassis is designed to allow for sturdy connection with the piston that is responsible for sliding the pack back and forth. Additionally, careful consideration is given to the width of the pitch pack to prevent the pack from becoming pinched by a compressed hull when the AUG is at depth, which would result in lack of glide angle controllability.

The payload pack is designed to fit around the central AUG electronic components, including the BSD computer, the IMU, and the main circuit board. Unlike the pitch pack, the payload pack is not dynamically positioned within the AUG body during the mission, so a minimal chassis design is used to maximize the room for the central electronic components. As a result of utilizing 3D printers for manufacturing the chassis, small adjustments can be 

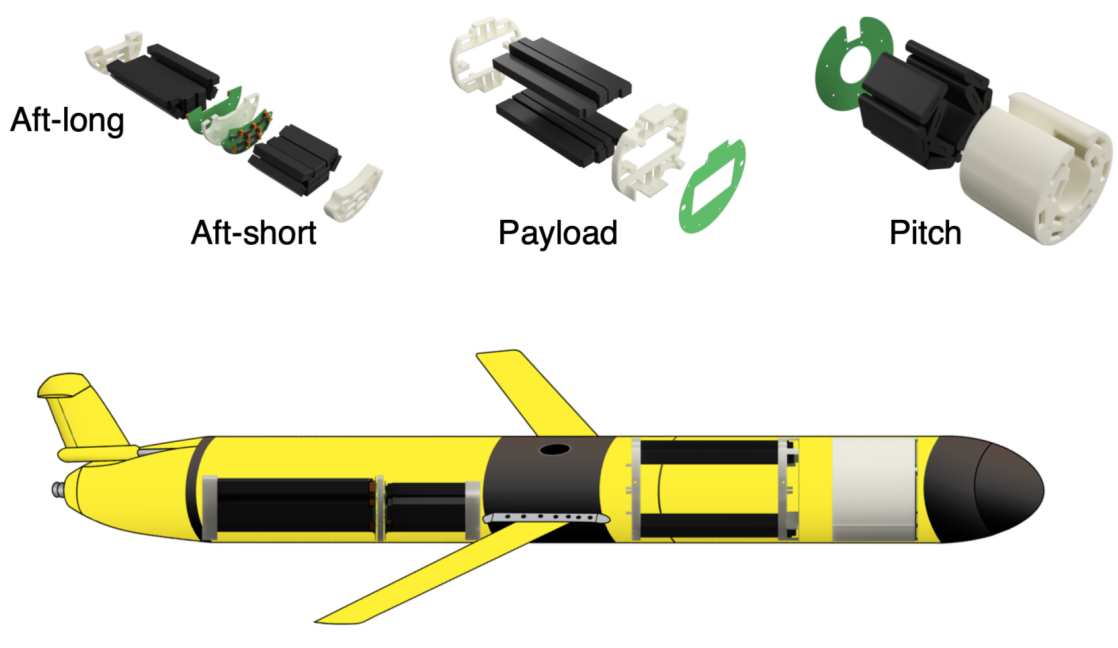

Figure 2-3: Battery pack configuration for proposed AUG design. The configuration includes four battery packs: the pitch pack, the payload pack, the aft-short pack and the aft-long pack. Lithium secondary batteries are shown in the diagram, but a similar design is possible for the higher energy density lithium primary batteries.

made such as the inclusion of gripper arms to improve battery stability while minimizing weight.

Similar to payload pack, the aft-short and aft-long packs do not dynamically change position within the AUG. The two aft battery packs are designed to be flush against the bottom of the AUG hull to accommodate the ballast pump that is positioned above the battery packs.

The lithium secondary battery packs are particularly useful for AUG operations because of their smart querying feature. Smart querying means that individual batteries amongst the four battery packs can be queried for voltage, current, percent charge, temperature, and desired charge rate. Additionally, individual batteries can be toggled on and off for both charging and discharging sequences. Having in-depth knowledge of battery state and precise control of battery maintenance enables better situational awareness for mission planning and greater efficiency when charging or discharging the batteries. By having centralized control of all batteries, selective charging can be performed when the AUG is on the bench without needing to disassemble the vehicle, enabling greater duty cycle for the AUG during research deployments.

The BSD computer can perform battery monitoring while the AUG is submerged similar 



Figure 2-4: Subplot A shows the graphical user interface (GUI) that is used for displaying battery state and charging the battery packs when the AUG is connected to a power supply while on the bench. Subplot B shows an example of battery state being tracked over time during a charge sequence, and Subplot $\mathrm{C}$ shows an example of the battery state GUI being used to monitor battery state when the AUG is connected to the bench. 
to the bench-top monitoring shown in Figure 2-4. This onboard awareness of battery state is particularly helpful for fault diagnosis purposes where it may be necessary to initiate contingency planning based on the remaining energy supply. Depending on the severity of the fault detected, vehicle recovery may be deemed infeasible and the AUG enters a state of data transmission to minimize data loss. Entering such a state is analogous to a Mars rover mission where instrument retrieval is not possible, but the transmitted data is still extremely valuable. However, with further improvements to cognitive robotics, the AUG may be able to enter a state of pseudo-hibernation while harvesting freely available environmental energy thus prolonging mission duration long enough for vehicle rescue, similar to Mark Watney, the character portrayed by Matt Damon in The Martian (2015), who resourcefully cultivated spuds using Martian soil and bio-waste to enable his possibility of returning to Earth.

\subsection{Onboard Acoustic Sensing}

Onboard acoustic sensing can provide several functionalities, including: navigation, mapping, and communication. In the context of unattended sea-ice survey in the Arctic, external aids such as surface vessels or landmark beacons are unavailable for acoustic communication. Furthermore, unattended underwater vehicles are solely responsible for performing exteroceptive sensing of environment state to enable active mission adjustments in response to said environment state.

Two acoustic sensors are considered in the proposed architecture: a Mechanical Scanning Imaging Sonar (MSIS) and Doppler Velocity Log (DVL). The MSIS is used for characterization of sea-ice and sea-state, while the DVL is used for navigation and estimating water column currents. The MSIS is located in the nose cone of the AUG and be rotated $360^{\circ}$ about the instrument's axis of rotation, thus forming a composite image from a series of acoustic scans. A gimbal mount is used within the AUG nosecone such that the MSIS axis of rotation is aligned with the horizontal plane rather than the AUG body. The DVL is mounted in a downward-facing orientation in the middle section of the AUG. The DVL has four profiling beams at $30^{\circ}$ offset from the normal direction of the transducer head, and each DVL beam measures the Doppler shift to estimate water-track and bottom-track velocities. The DVL is rigidly mounted to the AUG body such that the instrument axes move with the AUG axes. Figure 2-5 shows the acoustic beam-width for both instruments. 

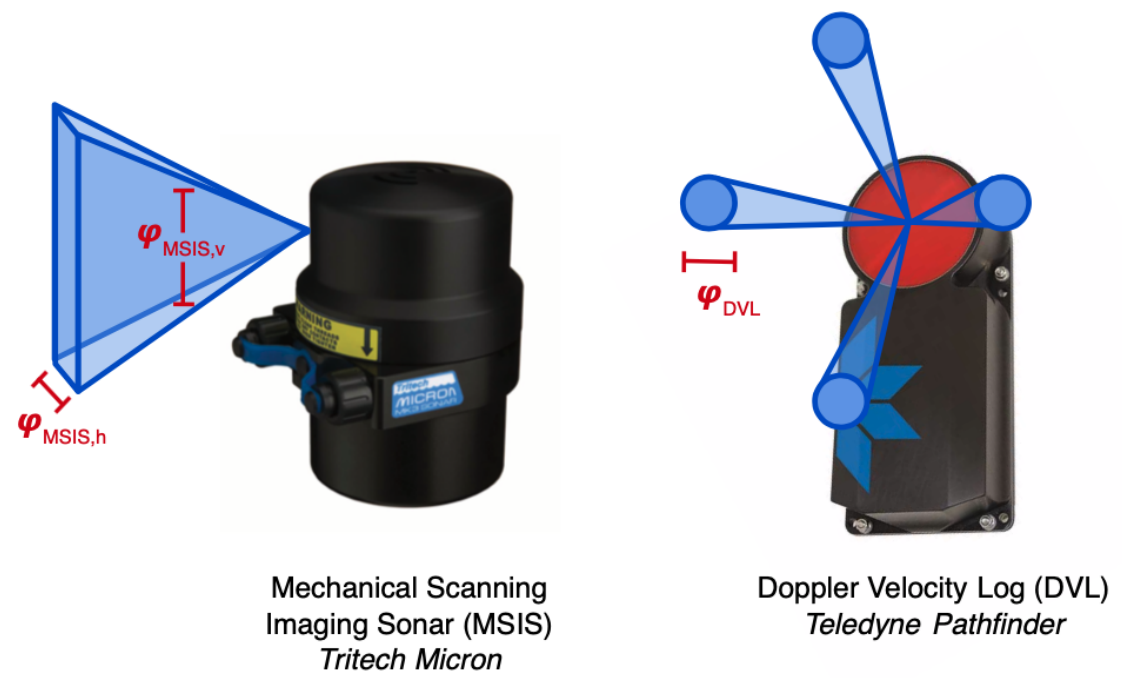

Figure 2-5: Acoustic beam width for the MSIS and DVL instruments. The MSIS beam width is given by two angles: $\varphi_{M S I S, h}=3^{\circ}$ is the horizontal beam width, and $\varphi_{M S I S, v}=$ $35^{\circ}$ is the vertical beam width. The DVL consists of four narrow profiling beams, each with beam width angle $\varphi_{D V L}=2.2^{\circ}$.

\begin{tabular}{|l|c|c|c|c|c|c|}
\hline & $\begin{array}{c}\text { Min Range } \\
{[\mathrm{m}]}\end{array}$ & $\begin{array}{c}\text { Max Range } \\
{[\mathrm{m}]}\end{array}$ & $\begin{array}{c}\text { Frequency } \\
{[\mathrm{kHz}]}\end{array}$ & $\begin{array}{c}\text { Beam Width } \\
{\left[{ }^{\circ}\right]}\end{array}$ & $\begin{array}{c}\text { Mass } \\
{[\mathrm{kg}]}\end{array}$ & $\begin{array}{c}\text { Power } \\
{[\mathrm{W}]}\end{array}$ \\
\hline Micron MSIS & 0.3 & 75.0 & 700 & 35,3 & 0.33 & 3.0 \\
\hline Pathfinder DVL & 0.2 & 89.0 & 600 & 2.2 & 1.19 & 2.0 \\
\hline
\end{tabular}

Table 2.3: Review of notable parameters for the MSIS and DVL instruments. Note that the maximum and minimum range listed for the DVL instrument corresponds to DVL operating in bottom-track mode. When the DVL is operating in water-track mode, the instrument range envelope is tapered.

Table 2.3 reviews other acoustic sensor parameters such as range, frequency, mass, and power. The minimum range of the sensors is dictated by the blanking distance of the acoustic source, and the maximum range is dictated by attenuation and absorption of the acoustic signal in the water column.

Control and interpretation of the MSIS and the DVL sensors serve as the foundation of this thesis. Specifically, Chapter 3 focuses on interpretation of MSIS data for making sea-ice characterizations, Chapter 4 focuses on interpretation of DVL data for improving AUG navigation, and Chapter 5 leverages the results discussed in the two previous chapters to derive a set of energy-optimal AUG control policies. By improving the state estimation and control methods of the AUG, the vehicle is more prepared for basin-scale in-situ survey 
of Arctic sea-ice.

\subsection{Review of Software Components}

To support sensing, planning, and control onboard the AUG, several software modules must operate together in a coordinated fashion on the AUG science computer, otherwise referred to as the Backseat Driver computer (BSD). To make coordination of a diverse set of computer programs and hardware components, a robotics middleware called Robotic Operating System (ROS) is utilized. A robotics middleware provides a set of software frameworks that allows for management of software packages, message-passing between components, and low-level control of devices. ROS is open source and widely adopted amongst a diversity of research communities. Other notable robotics middleware programs include Mission Oriented Operating Suite with Interval Programming (MOOS-IvP) [Benjamin et al., 2010] and Lightweight Communications and Marshalling (LCM) [Huang et al., 2010].

ROS employs a modular design approach such that individual software modules are responsible for specialized tasks, and these modules communicate with one another using pre-defined message formats. Individual software modules are refereed to as ROS nodes, and communication links between ROS nodes are called ROS topics. Each ROS topic supports a specific message type which is defined by a ROS message. For example, a Navigation node may publish pose information to a $A U G$ Localization topic, where the message passed to the topic contains the current time in seconds along with $x, y, z$ position in meters. The collection of ROS nodes and ROS that support robotics operations are referred to the ROS computation graph. The ROS computation graph for the proposed AUG architecture is shown in Figure 2-6.

Walking through Figure 2-6, the sonar_surface_classifier ROS node is responsible for data processing and controlling of the MSIS instrument, represented by the tritech_msis hardware component, and publishing sea-ice and sea-surface characterization information to the ice_classifications and wave_classifications ROS topics so that the BSD computer can react to the evolving environment state. Similarly, the dvl navigator ROS node is responsible for data processing of the DVL instrument, represented by the pathfinder_dvl hardware component, and publishing navigation updates and propulsion commands to the navigation_updates and propulsion_commands ROS topics so that the 


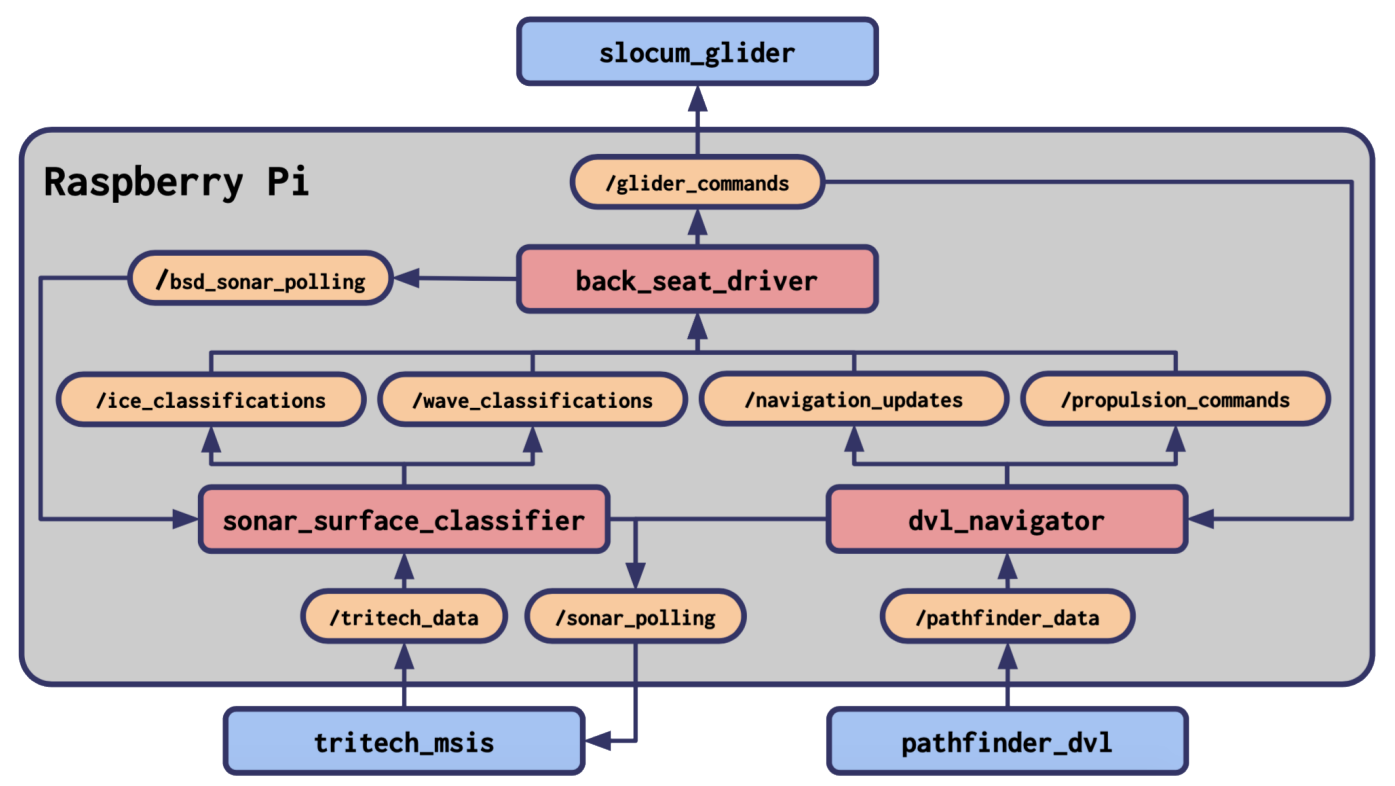

Figure 2-6: Robot Operating System (ROS) computation graph for the software components running the AUG's BSD computer, a Raspberry Pi. The red boxes are ROS nodes, the orange boxes are ROS topics, and the blue boxes are AUG hardware components. The figure highlights the connectivity between onboard sensor processes and AUG control processes.

BSD computer can react to the evolving vehicle state. The dvl_navigator ROS node also helps to control the MSIS instrument because the node is responsible for publishing AUG depth information, which impacts the range setting to be used by the MSIS.

The back_seat_driver node is responsible for ingesting all of the environment state and vehicle state updates from the MSIS and DVL interpretation nodes. Then, the BSD node is responsible for sending control commands to the AUG flight computer, which is represented by the slocum_glider hardware component. The AUG control commands then feed into the DVL navigation node to help perform odometry, a sub-component of navigation. Additionally, the BSD node is able to request MSIS control behavior via the bsd_sonar_polling topic. For example, if the BSD wants to initiate an AUG surfacing maneuver, it may first request a sequence of MSIS sea-ice and sea-surface scans to confirm that the environment state is conducive to safe surfacing.

The low-level details of the BSD node are outside the scope of this thesis, but a highlevel discussion of this node is provided in Section 2.7. The low-level details of the MSIS and DVL processing nodes are provided in Chapter 3 and Chapter 4, respectively. 


\subsection{Risk Aware Planning and Control}

Conducting in-situ sea-ice survey in the Arctic is inherently dangerous for underwater vehicles. Figure 2-7 provides a schematic overview of typical sea-ice features in the Arctic environment, showing how these features threaten vehicle safety.



Figure 2-7: Schematic diagram of an AUG conducting a sea-ice survey in the Arctic. The figure showcases how environment features make in-situ sea-ice survey dangerous for AUGs. The AUG is denied access to the ocean surface except for intermittent gaps between ice floes known as leads. Additionally, thick multi-year ice and pressure ridges serve as obstacles that the AUG must avoid when operating close to the sea surface. Figure adapted from [Jurohi, 2008].

As shown in Figure 2-7, sea-ice cover precludes the option for vehicle surfacing, decreasing the frequency that the AUG can receive mission updates, receive a new GPS fix, or transmit data. In the event that a serious fault is detected, the AUG cannot readily perform a mission abort and request an impromptu recovery operation from a shore-side support team. To make matters worse, even if the AUG was able to request a recovery operation, the rescue may be too dangerous to attempt, and the vehicle would be lost at sea.

In terms of sea-ice features serving as obstacles, multi-year ice and pressure ridges can be on the order of $10 \mathrm{~m}$ thick. Pressure ridges in particular can even impinge upon the seafloor in coastal areas. As a result, operations along the continental shelfs surrounding the Arctic, like the Chukchi Shelf for example, are particularly dangerous for AUG operations because seafloor depth is typically between $30 \mathrm{~m}$ and $100 \mathrm{~m}$, limiting the available of water column that is safe for operations.

Therefore, it is critical for the AUG to have access to robust risk-aware re-planning capa- 
bilities running onboard, or for the AUG to be provided a comprehensive set of contingency plans to fall back on should a conflict arise during the mission. This risk-aware planning and control capability is outside the scope of this thesis, but other researchers have focused more intensely on this problem. Previous efforts include probabilistic path planning under bounded risk [Ono et al., 2013], convex optimization for hybrid path and activity planning [Fernandez-Gonzalez et al., 2017], activity planning that is based on risk-reward tradeoff [Ayton and Williams, 2018], and goal-directed risk-bounded activity planning onboard AUVs [Timmons et al., 2016].

For the context of this thesis, it is assumed that a risk-aware planning system is operational onboard the AUG. This system is referred to as the Backseat Driver (BSD). The BSD is responsible for sending mission updates and control commands to the AUG as necessary. To enable informed decision making, the BSD utilizes state updates provided by the MSIS sea-ice characterization module and the DVL navigation module, both of which constitute the main contributions of this thesis.

\subsection{Summary and Future Work}

This chapter reviews the proposed AUG architecture in terms of hardware and software components, which is designed to enable basin-scale in-situ characterization of sea-ice. In doing so, this chapter motivates the need for onboard acoustic sensing for sea-ice characterization and navigation, as well as intelligent resource management to improve AUG energy efficiency.

Future work for specific software components will be discussed in more detail in the following chapters. For future work related to the AUG architecture, a formalized system for sea-ice and ocean current forecasting should be included. By having access to sea-ice and ocean current predictions, the mission planning system will be more capable of assessing mission risk and more prepared to develop contingency plans as necessary. Sea-ice modeling typically applies conservation equations of mass and momentum over the domain of interest to make a prediction regarding the underlying Ice Thickness Distribution (ITD) and Floe Size Distribution (FSD) functions [Hunke and Lipscomb, 2015, Feltham, 2008]. Accuracy of sea-ice models can be improved if coupled with atmospheric models and ocean circulation models. Similarly, ocean current modeling typically applies a reduced form of the Navier- 
Stokes equations over the domain of interest [Lermusiaux et al., 2006]. Uncertainty in both sea-ice and ocean current models can arise from uncertain boundary conditions, uncertain initial conditions, and uncertainty in the underlying physics. Additionally, high spatial or temporal resolutions can quickly lead to computational intractability. Moreover, to improve the proposed AUG architecture, a simplified but reliable sea-ice model and ocean current model should be included in the shore-side computation system so that local predictions of sea-ice and ocean-currents can be exploited by the AUG mission planner. 
This page intentionally left blank. 


\section{Chapter 3}

\section{Synoptic Sea-ice Mapping}

\subsection{Introduction}

Onboard acoustic sensing serves as the primary mechanism for AUVs to perform exteroceptive sensing of their environment. Significant efforts have been pursued in the realm of sonar-based mapping and characterization of oceanographic phenomena. In regards to acoustic-based mapping of the environment, a simultaneous localization and mapping (SLAM) approach that uses a probabilistic scan-matching routine to cross-register sequential scans recorded by an MSIS can be used to reconstruct a map of the underwater environment [Mallios et al., 2014]. This scan-matching method has demonstrated success in confined underwater cave environments [Mallios et al., 2016]. In the operational inverse of the underwater cave-mapping scenario, an MSIS has also been used for AUV iceberg mapping where the vehicle leverages a vehicle-attached occupancy map (VOM) representation to build a map of the iceberg [Zhou et al., 2019]. In terms of ice floe mapping, multibeam sonars have been mounted on AUVs to build digital elevation maps (DEMs) of the sea-ice cover and successfully distinguish between first-year sea-ice and multi-year sea-ice [Wadhams and Doble, 2008]. Similarly, multi-beam methods have been used in Antarctica to show that Antarctic sea-ice is characteristically thicker than what has been shown with ice-coring methods or satellite remote survey [Williams et al., 2015].

Aside from mapping efforts, onboard acoustics have been useful for characterization and detection of oceanographic phenomena as well. Of particular importance due to economic interest and risk of catastrophic environmental damage is the in-situ acoustic characterization and detection of submerged oil. By investigating the acoustical scattering properties 
associated with submerged droplets, it is possible to use broadband acoustics to quantify oil concentration and transport in the ocean [Loranger, 2019]. In the Arctic context, it has been shown that broadband acoustic backscatter techniques may be capable of detecting oil layers directly underneath the sea-ice cover when the sonar is operated with normal incidence with respect to the oil and sea-ice layer [Bassett et al., 2016]. Additionally, methods exist for estimating sea-ice thickness solely from acoustic backscatter, rather than considering freeboard altitude or keel depth [Bassett et al., 2020]. Thus, methods of characterization based on acoustic signature can be used in combination with sonar mapping techniques to establish a more detailed understanding of the environment.

For the AUG design, full coverage sea-ice mapping and acoustic characterization is not pursued as it would require constant operation of the scanning sonar, which would quickly deplete AUG resources (Note that more careful treatment of AUG resource management is presented in Chapter 5). Instead, intermittent sea-ice sensing using the MSIS instrument is proposed, where observations are made on the order of $50 \mathrm{~m}$ spatial intervals. This intermittent sea-ice sensing process is referred to as synoptic sea-ice mapping (SSIM). This Chapter develops an acoustic model for performing SSIM and applies SSIM to two ice observation datasets. Heavy emphasis is placed on the ability to discern thin first-year sea-ice from open water since this operational scenario is particularly challenging given the sensing modalities of the AUG and particularly important for the survivability of the AUG.

In SSIM, a three-tier classification scheme is used for describing sea-ice observations based on the following hierarchical factors: sea-ice presence, sea-ice thickness, and sea-ice roughness. This classification scheme is beneficial because it enables the use of multiple sensing modalities of the AUG, it concisely represents science data which improves the data transfer rate during AUG surfacing, and it promotes situational awareness of the onboard mission planning module.

\subsection{Properties of Sea-ice}

Sea-ice prevails in the Arctic at several different orders of magnitude in size, from mm sized ice crystals to $\mathrm{km}$ sized ice floes [Lucieer et al., 2016, Mei et al., 2019]. Properties of sea-ice at the smaller scale have more relevance for SSIM because sea-ice micro-structure dictates the acoustic scattering properties of the material. That said, large-scale sea-ice features 

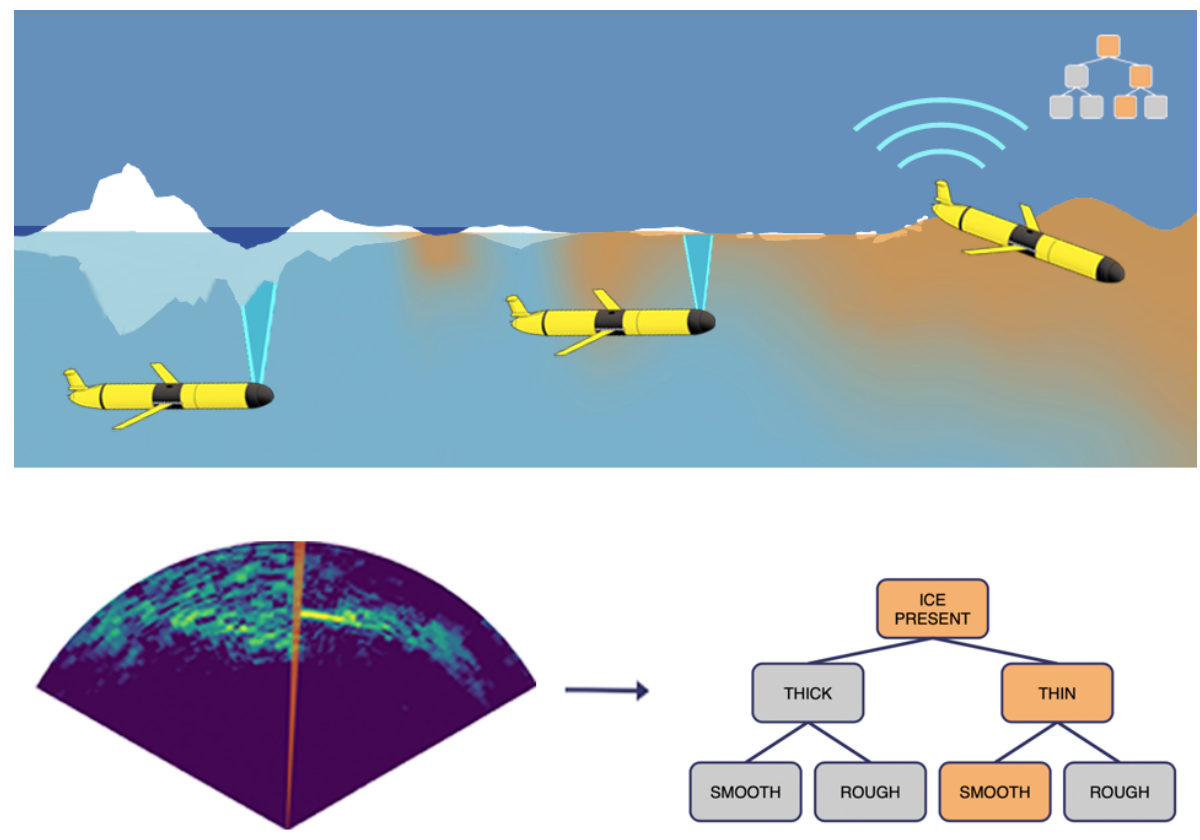

Figure 3-1: Schematic diagram of AUG sampling sea-ice in the Arctic and transmitting compressed sea-ice information via satellite communication. The onboard sensor processing module reduces raw acoustic measurements into hierarchical sea-ice features: sea-ice presence, sea-ice thickness, and sea-ice roughness. Figure adapted from [Lee et al., 2012].

such as pressure ridges are of critical importance for the navigation and mission planning systems [Wadhams and Doble, 2008].

Modeling all physically realizable components of sea-ice structure for the purpose of developing a detailed acoustics model is outside the scope of this thesis (The Community Ice CodE (CICE) manual provides a thorough overview of all aspects of sea-ice modeling [Hunke and Lipscomb, 2015]). Instead, this chapter focuses on capturing the general essence of seaice structure in order to perform SSIM with a scanning sonar. To do so, inhomogeneities introduced by brine channels that form during sea-ice formation are considered. Since salt cannot be incorporated into the crystalline structure that forms when water freezes, the salt is discarded from the material in a process known as brine rejection. This process leaves behind hollow columnar sections known as brine channels. These brine channels are known to contain bubbles which then act as scatterers when the sea-ice is ensonified by an acoustic signal [Bassett et al., 2020]. In addition to brine channel structures, another important sea-ice structural element is the vertically aligned congelation ice crystals that 


\begin{tabular}{|l|c|c|}
\hline & $\begin{array}{c}c \\
{\left[\mathrm{~m} \mathrm{~s}^{-1}\right]}\end{array}$ & $\begin{array}{c}\rho \\
{\left[\mathrm{kg} \mathrm{m}^{-3}\right]}\end{array}$ \\
\hline Air & 343 & 1.225 \\
\hline Seawater & 1450 & 910 \\
\hline Sea-ice & 3845 & 1028 \\
\hline
\end{tabular}

Table 3.1: Density and speed of sound for three mediums: air, seawater, and sea-ice. Seawater is assumed to have a temperature of $-2{ }^{\circ} \mathrm{C}$ and a salinity of $32 \mathrm{~g} \mathrm{~kg}^{-1}$. Density and speed of sound values for sea-ice are taken from [Timco and Frederking, 1996] and [Vogt et al., 2008], respectively.

form at the bottom of ice floes [Bassett et al., 2020]. These ice crystals lead to a complex ridging structure found at the bottom of sea-ice floes and are referred to as skeletal ice.

Along with structural aspects of sea-ice, bulk properties of sea-ice and the two adjacent mediums, namely air and seawater, are relevant for acoustic scattering processes as well. Table 3.1 reviews the density and speed of sound for air, seawater, and sea-ice. These bulk properties are crucial for understanding acoustic scatter intensities resulting from the interface of two mediums. Since the speed of sound difference between sea-ice and seawater is much greater than the density difference between the two mediums, the acoustic return intensity models are less sensitive to precise values for seawater and sea-ice density [Bassett et al., 2020]. Other notable properties of sea-ice, including mechanical properties, thermal properties, and electromagnetic properties, are discussed in [Schwarz and Weeks, 1977].

\subsection{Detection of Thin First-year Sea-ice}

During sea-ice survey operations, the AUG may use multiple sensing modalities to perform SSIM. Thick multi-year ice can readily be identified by examining the differential of the keel vertical range measured by the MSIS and the depth reported by the AUG pressure sensor. However, this depth-differential approach does not have the vertical resolution necessary for detecting thin first-year sea-ice. Here, thin sea-ice is defined as sea-ice that is $\leq 10 \mathrm{~cm}$ in thickness, which corresponds to the minimum thickness that can be reliably discerned using the depth-differential approach.

To overcome the challenge of detecting thin sea-ice, features of the acoustic signature that is induced by the properties of sea-ice are considered. First, the bubbles and other inhomogeneities that reside in the brine channels of sea-ice lead to internal scattering which elongates the acoustic signal received by the MSIS, increasing the peak-width of the signal. 
Next, the small-scale roughness of the sea-ice skeletal layer promotes Lambertian scattering (i.e. the surface scatters uniformly in all directions regardless of incidence angle) which enables meaningful acoustic returns at large incidence angles. Finally, the differences in speed of sound and density for the three mediums indicate that the seawater/air interface will lead to higher magnitude acoustic returns compared to the seawater/sea-ice interface. Figure 3-2 highlights how these features can be used to discern thin sea-ice from open water, including example acoustic scan-lines recorded during laboratory testing. Section 3.4 provides a more rigorous derivation of the classification scheme used for discerning thin sea-ice from free surface, Section 3.6 describes two experiments where the MSIS was used to observe ice and free surface conditions, and Section 3.7 describes the results of applying the SSIM classification scheme to the two datasets.

\subsection{Hierarchical Characterization of Sea-ice}

The AUG uses a tiered sea-ice characterization scheme based on three hierarchical features of sea-ice: sea-ice presence, sea-ice thickness, and sea-ice roughness. This classification scheme is beneficial because it enables the use of multiple sensing modalities of the AUG, it concisely represents science data which improves the data transfer rate during AUG surfacing, and it promotes situational awareness of the onboard mission planning module.

Sea-ice thickness is estimated by first measuring sea-ice keel depth by taking the difference between MSIS acoustic range and AUG pressure sensor depth, and then using the density difference between sea-ice and seawater to estimate sea-ice thickness. Sea-ice presence can readily be discerned for thick multi-year ice by inspecting the depth differential in keel vertical range and AUG depth. However, sea-ice presence is more challenging to assess for thin first-year sea-ice because the depth-differential does not have the vertical resolution to distinguish thin sea-ice from free surface. This section develops a scheme for performing SSIM with hierarchical sea-ice characterization, with emphasis on detecting thin first-year sea-ice.

First, the linear acoustic wave equation is derived from first principles, including: the equation of state (3.1), the conservation of mass (3.2), and the conservation of momentum (3.3). Here, $\mathbf{u}$ is the velocity vector in $\mathrm{m} \mathrm{s}^{-1} \rho$ is density in $\mathrm{kg} \mathrm{m}^{-3}, c$ is speed of sound in $\mathrm{ms}^{-1}$, and $p$ is pressure in $\mathrm{Pa}$. 

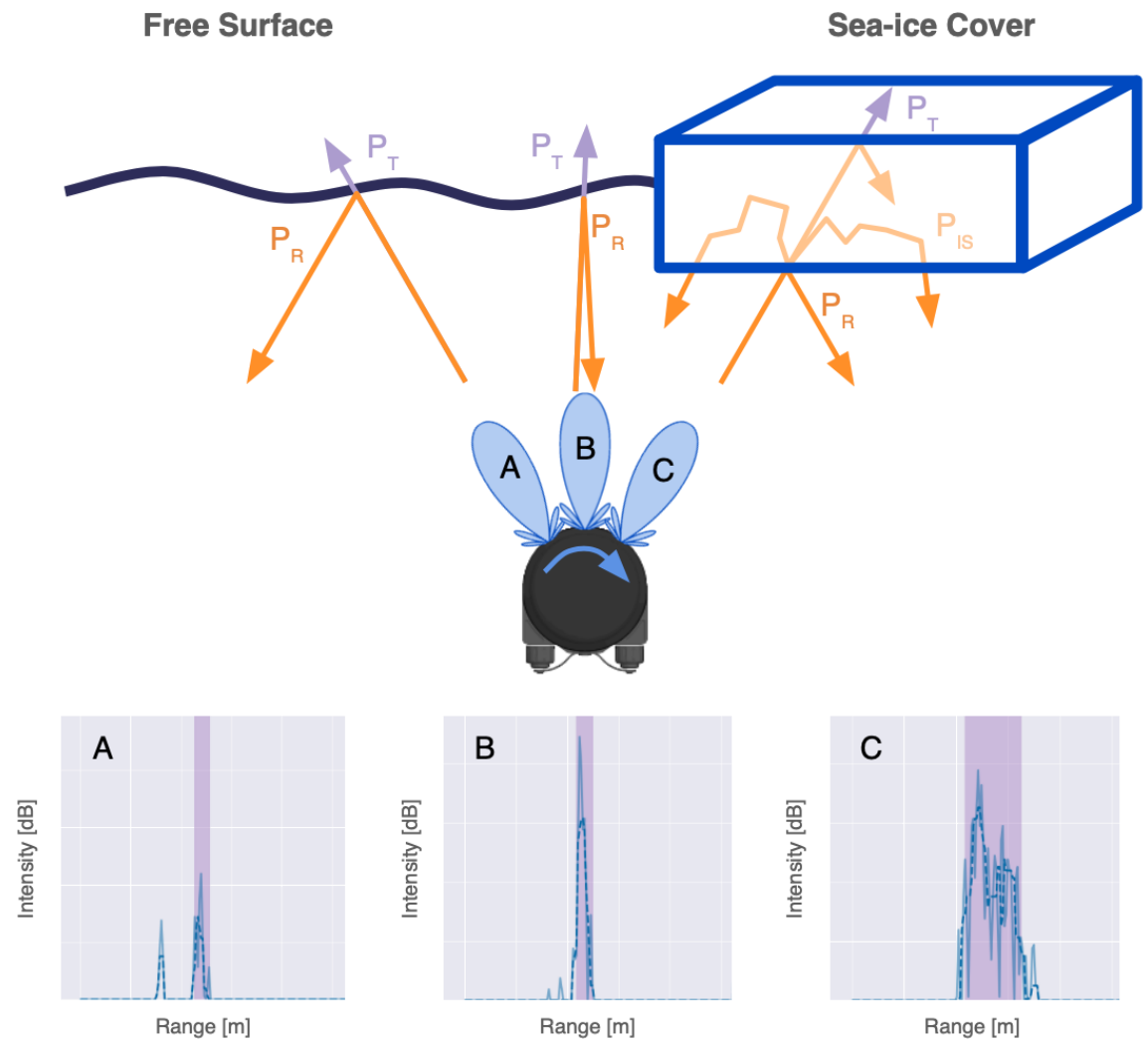

Figure 3-2: Schematic diagram of the MSIS sonar discerning thin sea-ice cover from free surface. $P_{R}, P_{T}$, and $P_{I S}$ represent the reflected, transmitted, and internal scattering acoustic waves, and together, they make up the components of acoustic scattering. The three subplots at the bottom of the figure show real acoustic scan-line data from laboratory testing that correspond with the bearings and surface conditions portrayed in the diagram (Note that the small initial peak in subplot A is caused by sidewall reflection in the test tank).

$$
\begin{aligned}
& \rho(p)=\left.\rho(p)\right|_{p=p_{0}}+\left.\left(p-p_{0}\right) \frac{\partial \rho}{\partial p}\right|_{p=p_{0}}+\left.\frac{\left(p-p_{0}\right)^{2}}{2 !} \frac{\partial^{2} \rho}{\partial p^{2}}\right|_{p=p_{0}}+\ldots \\
& \frac{\partial \rho}{\partial t}+\nabla \cdot(\rho \mathbf{u})=0 \\
& \rho \frac{\partial \mathbf{u}}{\partial t}+\rho \mathbf{u} \cdot \nabla \mathbf{u}=-\nabla p
\end{aligned}
$$

By considering only $0^{\text {th }}$ and $1^{\text {st }}$ order terms from the first principle equations, the linear 
acoustic wave equation can be derived, which is shown in Equation (3.4).

$$
\nabla^{2} p-\frac{1}{c^{2}} \frac{\partial^{2} p}{\partial t^{2}}=0
$$

The linear acoustic wave equation is a second order partial differential equation (PDE) that governs how acoustic waves propagate through a medium. One solution to the PDE of particular interest is the plane wave solution, which is shown in Equation (3.5). Here, $\mathbf{r}$ is the receiver location, $\mathbf{k}$ is the wave number, $\omega$ is the angular frequency of the wave, $t$ is time, $A$ is the wave amplitude, and $j$ is the imaginary number.

$$
p(\mathbf{r}, t)=A e^{j(\mathbf{k} \cdot \mathbf{r}-\omega t)}
$$

The plane wave is useful because it is simple and because it approximates arbitrary acoustic sources in the far-field. For example, a monopole source emits a spherical wave, but in the far-field, the spherical wave can be approximated as a plane wave.

When an acoustic wave transitions from one medium to another at a planar, Snell's law of refraction can be used, along with the continuity of pressure and the continuity of normal velocity, to determine the reflection and transmission behavior of the wave. Snell's law can be written as $\frac{\lambda_{1}}{\cos \theta_{1}}=\frac{\lambda_{2}}{\cos \theta_{2}}$, where $\lambda_{i}$ and $\theta_{i}$ are the wavelength and wave propagation angle with respect to the planar boundary of the $i^{\text {th }}$ medium. Both sides are multiplied by frequency $f$ to get $\frac{c_{1}}{\cos \theta_{1}}=\frac{c_{2}}{\cos \theta_{2}}$, where $c_{i}$ is the speed of sound for the $i^{\text {th }}$ medium.

Next, the incidence wave $P_{I}$ is related to the reflected wave $P_{S}$ and the transmitted wave $P_{T}$ via the reflection and transmission coefficients, $R$ and $T$.

$$
\begin{aligned}
P_{I}(\mathbf{r}, t) & =P_{0} e^{j\left(\mathbf{k}_{\mathbf{1}} \cdot \mathbf{r}-\omega t\right)} \\
P_{R}(\mathbf{r}, t) & =R P_{0} e^{j\left(\mathbf{k}_{\mathbf{1}} \cdot \mathbf{r}-\omega t\right)} \\
P_{T}(\mathbf{r}, t) & =T P_{0} e^{j\left(\mathbf{k}_{\mathbf{2}} \cdot \mathbf{r}-\omega t\right)}
\end{aligned}
$$

Now, the continuity of pressure and continuity of normal velocity are applied to solve for $R$ and $T$. Here, velocity $V$ is given by the following expression: $V=\frac{1}{j \omega \rho} \frac{\partial P}{\partial x}$. 


\begin{tabular}{|lll|r|c|}
\hline & & & \multicolumn{1}{c|}{$R$} & $T$ \\
\hline Air & $\rightarrow$ & Seawater & 1.0 & 2.0 \\
\hline Seawater & $\rightarrow$ & Air & -1.0 & 0.0 \\
\hline Air & $\rightarrow$ & Sea-ice & 1.0 & 2.0 \\
\hline Sea-ice $\rightarrow$ & Air & 1.0 & 0.0 \\
\hline Seawater $\rightarrow$ Sea-ice & 0.4 & 1.4 \\
\hline Sea-ice $\rightarrow$ Seawater & -0.4 & 0.6 \\
\hline
\end{tabular}

Table 3.2: Reflection and transmission coefficients for all acoustic transitions for the three mediums: air, seawater, and sea-ice. For this table, the incidence acoustic wave is assumed to be traveling normal to the boundary between the two mediums and that each medium is homogeneous.

$$
\begin{aligned}
& V_{I}(\mathbf{r}, t)+V_{R}(\mathbf{r}, t)=V_{T}(\mathbf{r}, t) \\
& P_{I}(\mathbf{r}, t)+P_{R}(\mathbf{r}, t)=P_{T}(\mathbf{r}, t)
\end{aligned}
$$

After plugging in the formula for velocity $V$, the two expressions in Equation (3.10) are used to solve for the $R(3.11)$ and $T$ (3.12) coefficients as a function of the density and speed of sound for both mediums, as well as the angle of incidence for the first medium $\theta_{1}$.

$$
R=\frac{\frac{\rho_{2}}{\rho_{1}} \sin \theta_{1}-\left[\left(\frac{c_{1}}{c_{2}}\right)^{2}-\cos ^{2} \theta_{1}\right]^{\frac{1}{2}}}{\frac{\rho_{2}}{\rho_{1}} \sin \theta_{1}+\left[\left(\frac{c_{1}}{c_{2}}\right)^{2}-\cos ^{2} \theta_{1}\right]^{\frac{1}{2}}}
$$

Although these equations do not consider inhomogeneities in the medium or non-planar interfaces between mediums, these equations help to establish an understanding for how the acoustic wave behaves at the various interfaces present in the AUG sea-ice survey scenario.

Table 3.2 shows $R$ and $T$ coefficients for all pairs of the three mediums given in Table 3.1. The incident wave is assumed to be traveling normal to the medium, $\theta_{1}=90^{\circ}$. As expected, the Table shows that the air $\rightarrow$ seawater and air $\rightarrow$ sea-ice interfaces act like rigid boundaries, whereas the seawater $\rightarrow$ air and sea-ice $\rightarrow$ air interfaces act like pressure-release boundaries. Of particular importance for discerning thin sea-ice cover from free surface is 
the fact that the seawater $\rightarrow$ sea-ice interface has lower acoustic reflectivity compared to the seawater $\rightarrow$ air interface. Although the transmitted wave from seawater to sea-ice will reflect off of the air and partially transmit back into seawater, this pathway yields a weaker acoustic intensity compared to the direct seawater $\rightarrow$ air reflection. This analysis is confirmed in [Bassett et al., 2020]. Therefore, based purely off of the bulk properties of the three mediums, it can shown that the free surface yields a higher acoustic return than the thin sea-ice cover.

Now, it is important to consider how irregularities in the sea-ice medium and microstructure of the sea-ice medium affect the acoustic signal received by the MSIS. First, to understand the effect of irregularities in the medium, the acoustic scattering equations and acoustic array theory are considered. The acoustic beam pattern $B(s)$ is given as a function of $s=\sin \theta$, and the taper function $T(u)$ is given as a function of $u=\frac{z}{\lambda}$. The beam pattern shows how the acoustic wave propagates into the medium, and the taper function represents an infinitesimal point source along the array transducer (or on the surface of the scattering object). Functions $B$ and $T$ are Fourier transform pairs of each other.

$$
\begin{aligned}
& B(s)=\int_{-\infty}^{\infty} T(u) e^{j 2 \pi s u} d u \\
& T(u)=\int_{-\infty}^{\infty} B(s) e^{-j 2 \pi s u} d s
\end{aligned}
$$

As a sanity check, it can be easily verified that a taper function equal to the delta function leads to uniform beam pattern for all angles $\theta$, which is consistent with the case of a monopole source. Similarly, an infinite taper function with uniform intensity leads to a delta function as the beam pattern, which is consistent with the plane wave case. For finite taper arrays, as is true with real world acoustic sensors, the Fourier integration yields a sinc function, where $\operatorname{sinc}(x)=\frac{\sin (x)}{x}$. The main peak of the sinc function corresponds with the main lobe of the acoustic beam pattern, while the local extrema of the sinc function correspond with the side lobes of the beam pattern.

When the Kirchhoff Approximation is made, which assumes locally planar reflections, finite acoustic targets behave the same way as finite acoustic sources: the finite targets yield a sinc-shaped acoustic reflection. When an acoustic wave encounters a field of inhomogeneities, as is the case with bubbles present in the sea-ice brine channels, this is 
called volume scattering and the effects of attenuation and dispersion must be considered. Together, attenuation and dispersion lead to a complex internal scattering process, which for the purposes of sea-ice survey, lead to elongated acoustic signals received by the MSIS [Bassett et al., 2020]. Therefore, the increased peak-width from internal scattering in the sea-ice layer serves as the second feature that allows thin sea-ice to be discerned from the free surface.

Now it is important to consider the external surface scattering that results from the ridged skeletal layer of sea-ice. The skeletal layer consists of vertically aligned congelation of ice crystals that form at the bottom layer of sea-ice. However, exact scattering equations for non-trivial geometries are mathematically challenging, or even intractable, to derive. For example, even the acoustic scattering of a fluid sphere is rather mathematically involved [Anderson, 1950, Sullivan-Silva, 1989]. Instead of pursuing an exact acoustic model given the expected structure of skeletal ice, a dimensionless scatter function $S$ is introduced for the material. This allows for the use aggregate statistics to model the complicated and intricate geometries. The scatter function is dependent on four angles, the azimuth and zenith angles of the incident acoustic wave with respect to the target surface normal $\omega_{i}=\left(\phi_{i}, \theta_{i}\right)$, as well as the azimuth and zenith angles of the receiver with respect to the target surface normal $\omega_{r}=\left(\phi_{r}, \theta_{r}\right)$. The far-field acoustic scatter function can be written as a function of $S$, as shown in Equation (3.15).

$$
P_{s}(\mathbf{r})=P_{0}\left(\frac{e^{j k r}}{r}\right)\left[\frac{S\left(\omega_{i}, \omega_{r}\right)}{k}\right]
$$

The form of $S$ dictates the how the material responds to acoustic excitation at different angles. This modeling approach is identical to the Bidirectional Reflectance Distribution Function (BRDF) from the optics community [Nicodemus, 1965]. Figure 3-3 shows the qualitative behavior of different scatter functions $S$, which is herein referred to as the Bidirectional Scattering Distribution Function (BSDF).

As a result of the micro-ridging structure at the bottom skeletal layer, sea-ice expresses Lambertian scattering behavior, meaning that the sea-ice surface yields uniform acoustic scattering independent of incidence angle. Therefore, the ability to receive acoustic returns at large angles of incidence serves as the third acoustic feature that allows thin sea-ice cover to be discerned from free surface. 




Figure 3-3: Examples of BSDF function $S$ for different surfaces. Note that only the reflected wave is portrayed in figure for simplicity. Surface A portrays a mirror-like surface that exhibits a planar wave reflection, Surface B portrays a glossy surface that exhibits a specular wave reflection, and Surface C portrays a Lambertian surface that exhibits uniform reflection in all directions. Figure adapted from [VonHaarberg, 2018].

In conclusion, the SSIM strategy includes a hierarchical characterization of sea-ice that considers three primary attributes: sea-ice presence, sea-ice thickness, and sea-ice roughness. Sea-ice thickness and roughness can be assessed via the combination of keel vertical range and AUG depth. However, assessing the presence of sea-ice, namely thin first-year sea-ice, requires further treatment of the received acoustic signal. Based on first-principle acoustics equations and the known properties of sea-ice, three attributes of the acoustic signal are suggested that can allow thin sea-ice the be discerned from free surface: increased peakwidth, increased incidence angle, and decreased maximum intensity. These attributes are portrayed in Figure 3-4, where the figure displays acoustic data collected during tank testing in Woods Hole, MA.

\subsection{Active Sensing}

In this section the SSIM problem is considered from the active sensing perspective: how can the MSIS be actuated to achieve the sea-ice observations that are desired, and how can actuation be adapted to the the current vehicle and environment state? Of course SSIM also depends on AUG control, but this is outside the scope of this thesis. Active sensing is discussed in greater detail in Chapter 6 as a future work item.

For now, four control parameters pertaining to the actuation of the MSIS instrument are considered: range $r_{M S I S}$, gain $k_{M S I S}$, bearing $\boldsymbol{\psi}_{M S I S}$, and angular resolution $\dot{\boldsymbol{\psi}}_{M S I S}$. Note that angular resolution $\dot{\boldsymbol{\psi}}_{M S I S}$ encodes the difference in bearing between successive MSIS measurements, so high resolution scanning corresponds with small values of $\dot{\boldsymbol{\psi}}_{M S I S}$. Due to the gimbal mounting mechanism used onboard the AUG, the MSIS axis of rotation 




Figure 3-4: Array of acoustic scan-lines for both ice and water surface conditions, for $5^{\circ}$, $15^{\circ}$, and $25^{\circ}$ incidence angles. High intensity peaks that appear before and after the $0.4 \mathrm{~m}$ range are caused by sidewall and surface reflections that arise from the confined test tank environment that was used during data collection. Note that the data was collected in a freshwater environment.

is parallel to the sea-ice surface, effectively decoupling instantaneous AUG pitch from the SSIM problem.

The range and gain setting can be controlled in response to the vertical distance between the MSIS transducer head and the sea-ice keel. This vertical distance is referred to as the keel offset. By adjusting the range and gain setting in response to keel offset, the transmission loss of the acoustic signal can be accounted for. Equation (3.20) gives the simple sonar equation, where $L_{p}$ is the sound pressure level, $L_{s}$ is the source level, $H_{i}$ is the transmission loss of the incident wave, $H_{s}$ is the transmission loss of the scattered wave, and $T_{s}$ is the target strength of the material. 


$$
\begin{aligned}
L_{p} & =10 \log _{10}\left(\frac{|p|^{2}}{\left|p_{r e f}\right|^{2}}\right) \mathrm{dB} \text { re } p_{\text {ref }} \\
L_{s} & =10 \log _{10}\left(\frac{A^{2}}{r_{r e f}^{2} p_{r e f}^{2}}\right) \mathrm{dB} \text { re } r_{r e f}, p_{r e f} \\
H & =10 \log _{10}\left(\frac{r^{2}}{r_{r e f}^{2}}\right) \mathrm{dB} \text { re } r_{r e f} \\
T_{s} & =10 \log _{10}\left(\frac{|S|^{2}}{\left|r_{r e f} k\right|^{2}}\right) \mathrm{dB} \text { re } r_{r e f} \\
L_{p} & =L_{s}-H_{s}-H_{i}+T_{s}
\end{aligned}
$$

Next, the bearing and angular resolution can be adjusted as a function of the sea-ice property currently being estimated, between sea-ice presence, sea-ice thickness, and sea-ice roughness. Detecting sea-ice presence is dependent on the thickness level. For the more challenging case of thin first-year sea-ice, the MSIS is commanded to observe a large swath of $\pm 60^{\circ}$ incidence relative to the sea surface at high angular resolution. Large incidence angles must be considered during the sea-ice detection process because angle of incidence is one of the key acoustic properties that allows sea-ice to be discerned from free surface. Next, to measure sea-ice thickness, only a narrow bearing window $\pm 10^{\circ}$ must be considered at high resolution. Finally, to measure sea-ice roughness, a moderate bearing window of $\pm 30^{\circ}$ must be considered at high resolution, which allows variation in thickness to be assessed without introducing distortion effects that occur at large incidence angles.

In this thesis, wave characterization in the Marginal Ice Zone (MIZ) is not considered. However, characterizing waves requires a different active sensing strategy for the MSIS instrument: the use of low angular resolution over a small bearing window. This sensing strategy allows for the measurement of the Doppler shift of the traveling wave, and when multiple successive wave characterizations are made, the wave propagation direction can be deduced [Burgess et al., 2020]. As discussed in Chapter 2, having knowledge of wave properties in the MIZ is helpful both for science purposes but also for adaptive mission planning carried out by the BSD computer. A summary of the different sensing modes is shown in Table 3.3. Further discussion on MSIS actuation is provided in Chapter 5 in the context of hotel load minimization and energy efficiency. 


\begin{tabular}{|l|c|c|}
\hline \multicolumn{1}{|c|}{ Sensing Mode } & $\begin{array}{c}\text { Bearing } \\
\boldsymbol{\psi}_{M S I S}\end{array}$ & $\begin{array}{c}\text { Resolution } \\
\dot{\boldsymbol{\psi}}_{M S I S}\end{array}$ \\
\hline Sea-ice Presence & $\pm 60^{\circ}$ & High \\
\hline Sea-ice Thickness & $\pm 10^{\circ}$ & High \\
\hline Sea-ice Roughness & $\pm 30^{\circ}$ & High \\
\hline
\end{tabular}

Table 3.3: Review of MSIS actuation strategies in terms of instrument bearing and angular resolution for different sensing modes: sea-ice presence, sea-ice thickness, and sea-ice roughness.

\subsection{Experiments}

Two sets of SSIM experiments were conducted with the MSIS. First, Subsection 3.6.1 reviews a set of laboratory tank tests in Woods Hole, MA. Then, Subsection 3.6.2 reviews a set of field deployments in Saint-Fabien, QC. After the experiments are introduced, the results of SSIM are discussed in Section 3.7.

\subsubsection{Laboratory Tests in Woods Hole}

During laboratory testing in Woods Hole, MA, the MSIS was suspended in an outdoor test tank at $42 \mathrm{~cm}$ depth. Half of the tank surface was ice-free and the other half of the tank was covered with a $7.25 \mathrm{~cm}$ thick layer of ice. The MSIS was mounted in the center of the tank to minimize the effect of side-wall reflections. The tank was filled with freshwater, and as a result, the ice in the tank did not have a brine channel structure that was described in Section 3.2. That said, the ice contained frozen air bubbles which act as internal scatterers in the ice slab, and the bottom layer of the ice structure had a characteristic micro-structure that was qualitatively similar in roughness compared to the skeletal layer of sea-ice. Although the conditions in the tank test are quite different than what is expected of the ice-surveying AUG in the Arctic, this data-set helps provide a proof-of-concept for the ice detection process as part of the SSIM module. Figure 3-5 shows the experimental setup of the tank test.

To accommodate the shallow mounting depth of the MSIS, the minimum range setting of $2 \mathrm{~m}$ was utilized. Recall that the minimum detectable range for the MSIS is $30 \mathrm{~cm}$, which is derived from the blanking distance of the sonar. Also, to avoid over-saturation of the acoustic signal, a low gain setting was used as well. After measurements were taken when the tank surface was evenly split between ice-free and ice-covered area, small ice wedges 


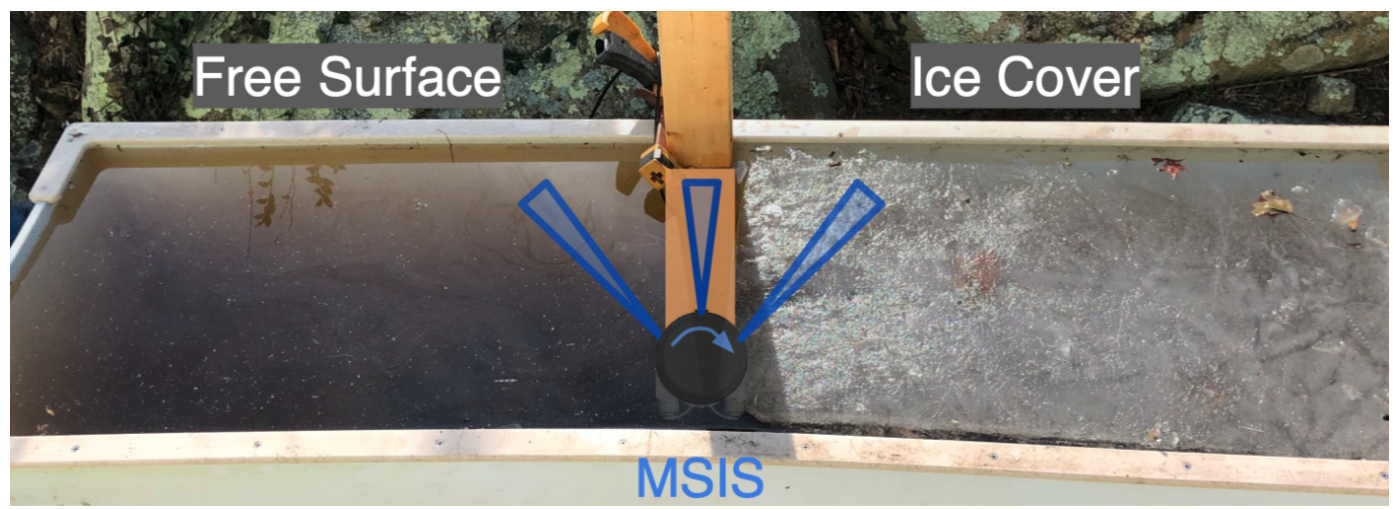

Figure 3-5: Experiment setup for the laboratory tank testing with the MSIS in Woods Hole, MA. The figure shows the MSIS sonar mounted at a water depth of $42 \mathrm{~cm}$ and centered at the interface of free surface and ice cover. The ice was measured by hand to be $7.5 \mathrm{~cm}$ thick.

were added to the ice-free area. In doing so, the Percent Ice Cover (PIC) was modulated on one side of the test tank, which allows for investigation of the effect of PIC on the received acoustic signal. Interestingly, even a small PIC was observed to have a large effect on the acoustic signature of the sonar swath, making ice presence readily detectable even in the case of partial ice coverage. This observation is particularly helpful from an AUG safety perspective because it helps minimize the chances that the AUG attempts a surfacing maneuver in a potentially dangerous partial ice-cover situation. Figure 3-6 showcases how the PIC was modulated during the tank test experiments.

\subsubsection{Field Deployments in Saint-Fabien}

During field deployments in Saint-Fabien, QC, the MSIS was deployed in various sea-ice conditions within Baie du Ha! Ha!, a small bay on the Saint Lawrence River. The survey site in Saint-Fabien is located at $\left[48.3^{\circ} \mathrm{N}, 68.8^{\circ} \mathrm{W}\right]$. Figure $3-7$ shows the testing locations of the four-day research deployment in Saint-Fabien.

The sea-ice in Baie du Ha! Ha! was accessed via ice canoe. Ice canoeing originally served as a means of transport between the shores of the Saint Lawrence River, but now ice canoeing is considered a sport. However, since ice canoeing provides a great deal of mobility amongst a harsh landscape of ice floes and pressure ridges, ice canoes are commonly used as vehicles for research as well. Some members of the Saint-Fabien operations team are shown in Figure 3-8 as they prepare their scientific equipment for various experiments. Behind 




Figure 3-6: Percent Ice Cover (PIC) variation during laboratory tank testing in Woods Hole, MA. The left column of the figure shows the raw camera images of the various PIC conditions, while the right column of the figure shows the ice wedges highlighted in red for ease of interpretation.

the canoe, I can be seen constructing the wooden mount that was used to hold the MSIS in place while recording sea-ice observations. Since space is limited onboard the ice canoe, only essential equipment can be carried during deployment.

After reaching a suitable science location, the crew can stand up on the ice floe and assemble their equipment. For in-water sampling instruments like the MSIS sonar, a hole must first be cut into the ice cover using an ice saw to enable access to the underwater environment below. Figure 3-9 showcases one of the holes that was cut in the sea-ice in order to enable data collection. During the Saint-Fabien deployment, the sea-ice was observed to be between $25 \mathrm{~cm}$ and $30 \mathrm{~cm}$ thick.

Similar to the varying PIC data-set collected in the laboratory, a data-set of varying PIC conditions was collected during the Saint-Fabien deployment as well. To produce varying 


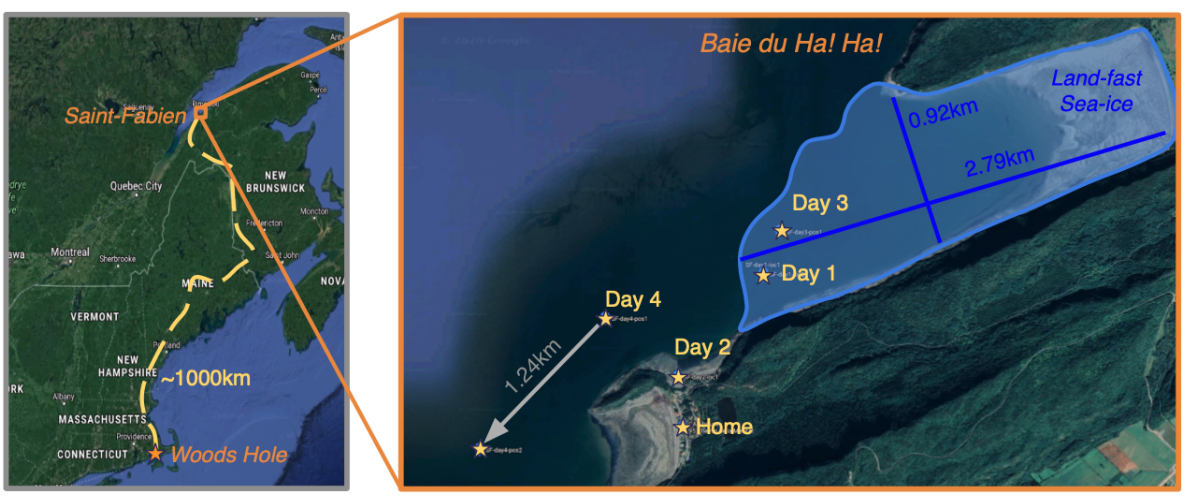

Figure 3-7: Field deployment in Saint-Fabien, QC. The deployment spanned four consecutive days, where the sampling location for each day is indicated by a gold star in the right subplot. The light-blue highlighted area shows the approximate portion of the bay that was covered with land-fast sea-ice for the duration of the deployment. Outside the land-fast sea-ice zone, marginal sea-ice cover was observed.

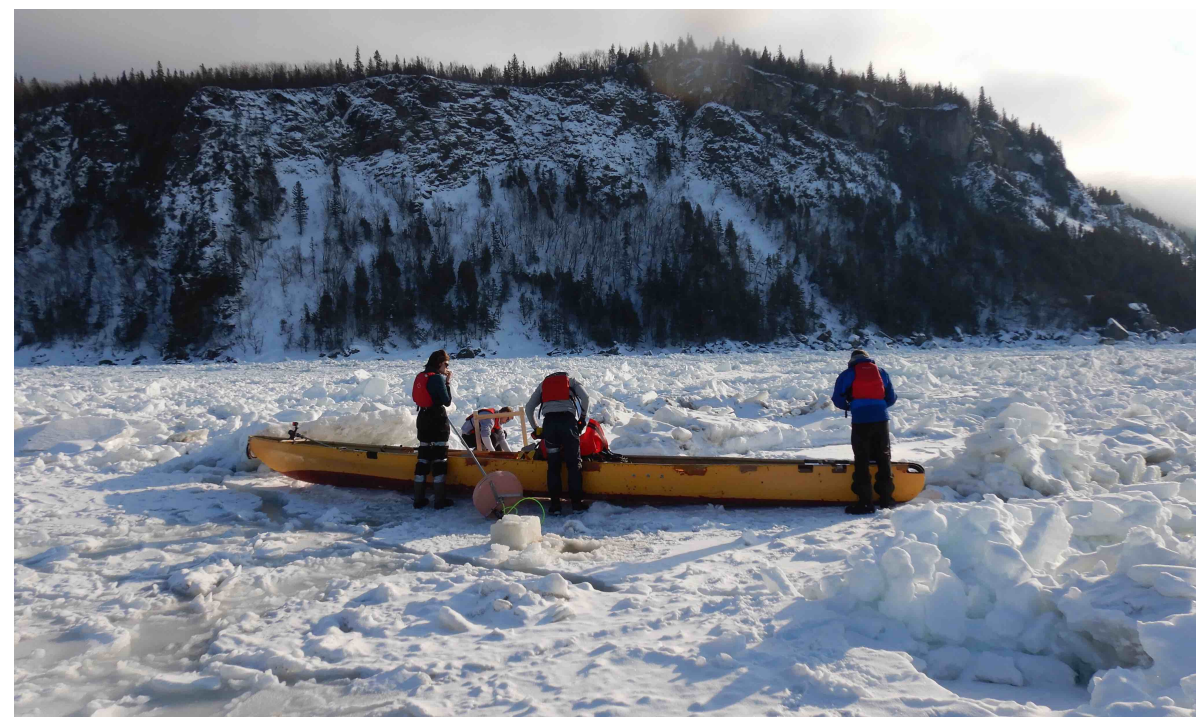

Figure 3-8: Ice canoeing in Baie du Ha! Ha!, a small bay in Saint-Fabien, QC, situated on the Saint Lawrence River. Operated by a team of five people, the ice canoe is sledged over ice floes and pressure ridges, and paddled across sections of open water. Only a limited amount of scientific equipment may be deployed in the field because all equipment must be carried onboard the canoe.

PIC conditions during the field deployment, small sea-ice wedges were carefully removed from the area directly above the MSIS instrument. The varying PIC conditions are shown in Figure 3-10.

Table 3.4 shows the PIC values for both the Woods Hole tank test data and the SaintFabien field deployment data, shown in Figure 3-6 and Figure 3-10 respectively. 


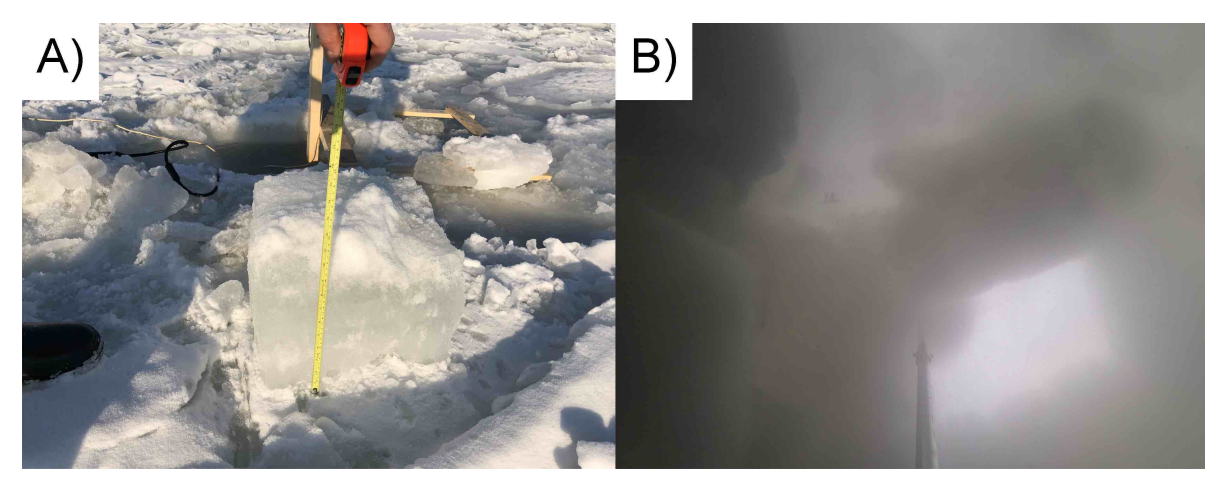

Figure 3-9: Preparation for data collection during field deployments in Saint-Fabien, QC. Sub-figure A shows a hole that was cut into the ice cover using an ice-saw so that under-ice MSIS sonar samples can be recorded. Sub-figure B shows the view from underneath the sea-ice cover, captured using a waterproof GoPro camera.

\begin{tabular}{|c|c|c|c|c|c|c|c|}
\hline \multicolumn{6}{|c|}{ Laboratory Testing in Woods Hole, MA } \\
\hline Name & A & B & C & D & E & F & G \\
\hline PIC [\%] & 8.7 & 14.6 & 25.0 & 37.2 & 48.9 & 73.6 & 88.3 \\
\hline
\end{tabular}

\begin{tabular}{|c|c|c|c|c|c|c|c|}
\hline \multicolumn{7}{|c|}{ Field Deployment in Saint-Fabien, QC } \\
\hline Name & A & B & C & D & E & F & G \\
\hline PIC [\%] & 36.3 & 50.6 & 60.6 & 66.0 & 74.6 & - & - \\
\hline
\end{tabular}

Table 3.4: Percent Ice Cover (PIC) for two data-sets: a laboratory test data-set recorded during tank test operations in Woods Hole, MA, and a field deployment data-set recorder during ice-canoe operations in Saint-Fabien, QC.

Although the data collected during the Saint-Fabien deployment is not extensive in terms of exposure to a variety of sea-ice conditions, or representative of the acoustic samples that the AUG would collect during sea-ice survey due to limited range offset, the data is instrumental in the development of the SSIM module. The dataset provides key insights regarding the acoustic properties of sea-ice and helps to inform future design decisions for Polarsentinel.

\subsection{Results}

For results of the SSIM module, sea-ice detection serves as the primary focus. To perform sea-ice detection, a binary classifier was employed based on the three acoustic features described in Section 3.4: peak width, incidence angle, and maximum intensity. For the binary classifier, sea-ice cover is considered the positive condition and free-surface is considered 


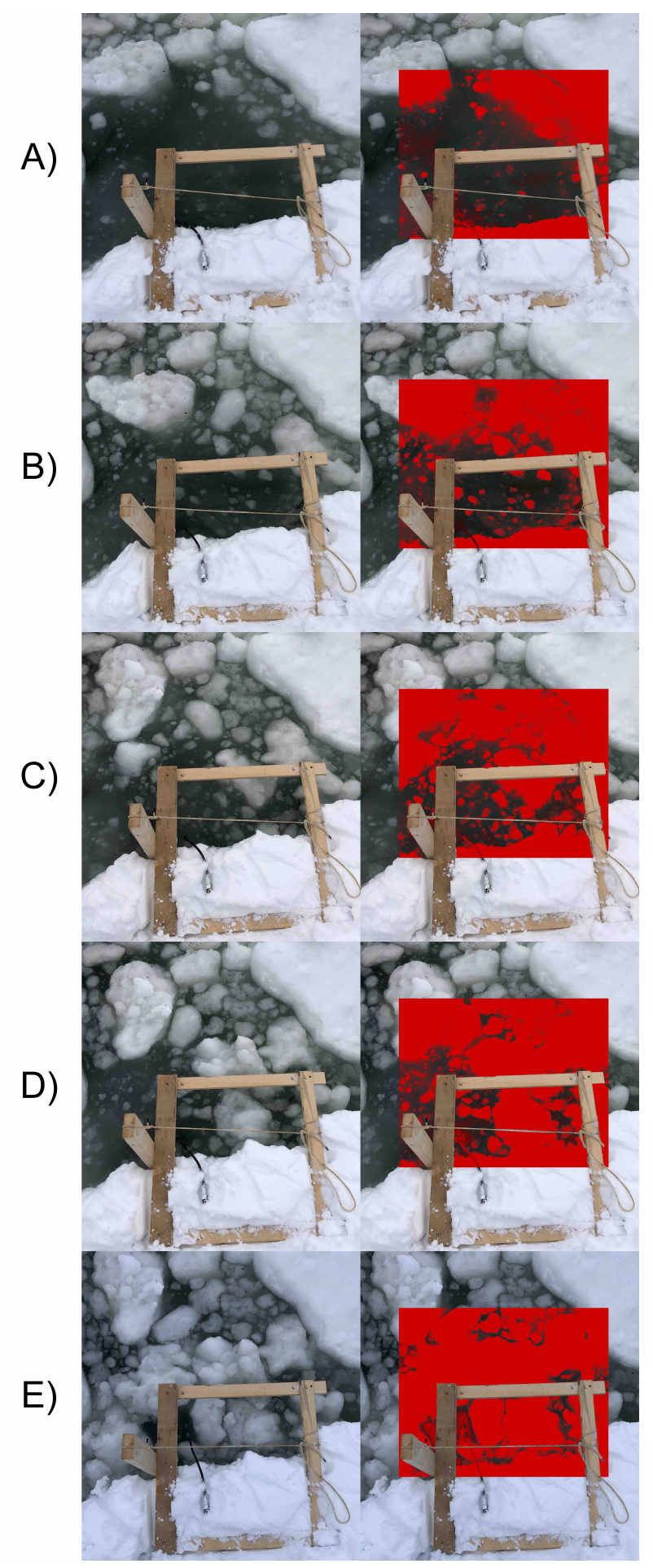

Figure 3-10: Percent Ice Cover (PIC) variation during field deployment testing in SaintFabien, QC. The left column of the figure shows the raw camera images of the various PIC conditions, while the right column of the figure shows the ice wedges highlighted in red for ease of interpretation. 
the negative condition. The performance of the binary classifier is evaluated using a Receiver Operating Characteristic (ROC) curve. ROC curves are helpful for evaluating the performance of a binary classifier because they encode both error conditions: False Positives (Type I error) and False Negatives (Type II error). Since ice cover is defined as the positive condition, a false positive occurs if SSIM detects ice when no ice is present, and a false negative occurs if the SSIM detects free surface when ice is present.

\subsubsection{Feature Extraction}

Before performance of the SSIM ice detection scheme is assessed, the result of feature extraction are shown with respect to the three sea-ice acoustic features: peak width, incidence angle, and maximum intensity. Figure 3-11 displays the sea-ice features for the acoustic data collected during laboratory tests in Woods Hole, and Figure 3-12 displays the sea-ice features for the acoustic data collected during field deployments in Saint-Fabien.

Peak width of the acoustic signal is extracted using the Full Width Half Maximum (FWHM) method. FWHM assesses the width of a signal by considering the distance between two points on the signal that are half of the maximum signal amplitude. Next, the maximum intensity is easily extracted from the acoustic scanline by taking a maximum over the array of intensities, and the angle of incidence is calculated from the current bearing angle of the MSIS. Note that the acoustic intensity of the MSIS includes a time variable gain (TVG), which corrects for signal loss due to attenuation and spreading.

Although the laboratory dataset is considerably more dense than the field deployment dataset, both datasets exhibit the same trends. Relative to free-surface, sea-ice cover tends to correlate with large peak-widths, strong acoustic return at large incidence angles, and weak acoustic return at small incidence angles. These patterns are consistent with the acoustic equations discussed in Section 3.4. The fact that both datasets express the same trends with regards to the acoustic features of sea-ice despite differing operating conditions is a testament to the generality of the SSIM ice detection approach.

\subsubsection{Sea-ice Detection}

Using the features displayed in Subsection 3.7.1, a binary classifier can be constructed that outputs whether or not sea-ice is present based on acoustic observation as input. The performance of the binary classifier is evaluated using ROC curves, where the $\mathrm{x}$-axis shows 


\section{Sea-ice Detection: Woods Hole Tank Test}
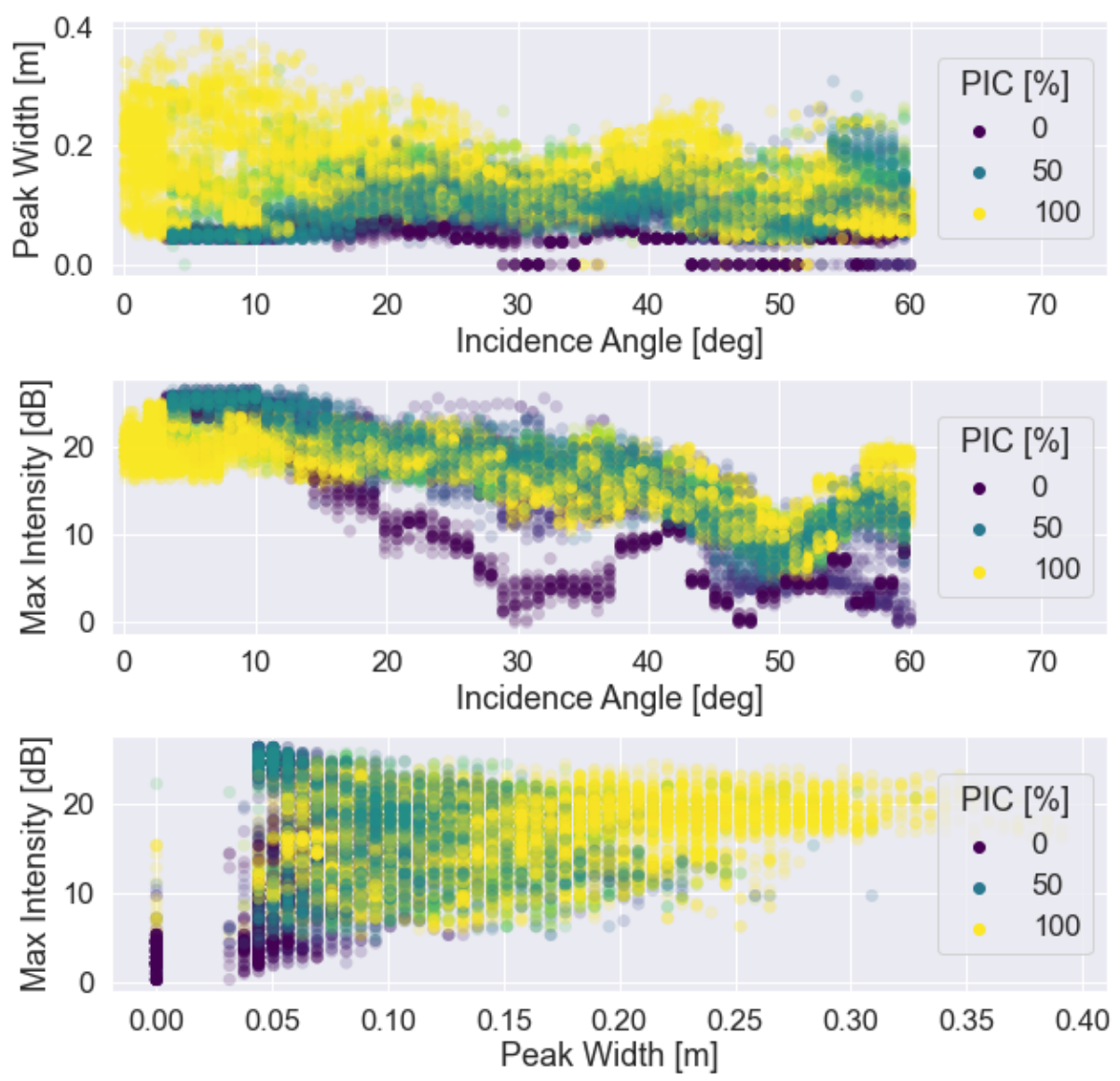

Figure 3-11: Acoustic features of sea-ice for laboratory data collected in Woods Hole, MA. Peak-width, incidence angle, and maximum intensity are plotted as 3D coordinates in threeview format. The color of each point indicates percent ice cover (PIC), where dark blue denotes free-surface, yellow denotes full ice cover, and green or purple colors indicate partial ice cover.

the False Positive Rate (FPR) and the y-axis shows the True Positive Rate (TPR) of the classifier. In this context, a True Positive outcome signifies that the classifier correctly identifies the presence of sea-ice, and a False Positive outcome signifies that the classifier incorrectly identifies the presence of sea-ice. As such, the perfect classifier resides at point $(0,1)$, correctly identifying the presence of ice $100 \%$ of the time, and falsely identifying sea-ice as water $0 \%$ of the time.

To evaluate the effectiveness of the feature-based approach of sea-ice detection, de- 


\section{Sea-ice Detection: Saint-Fabien Field Deployment}
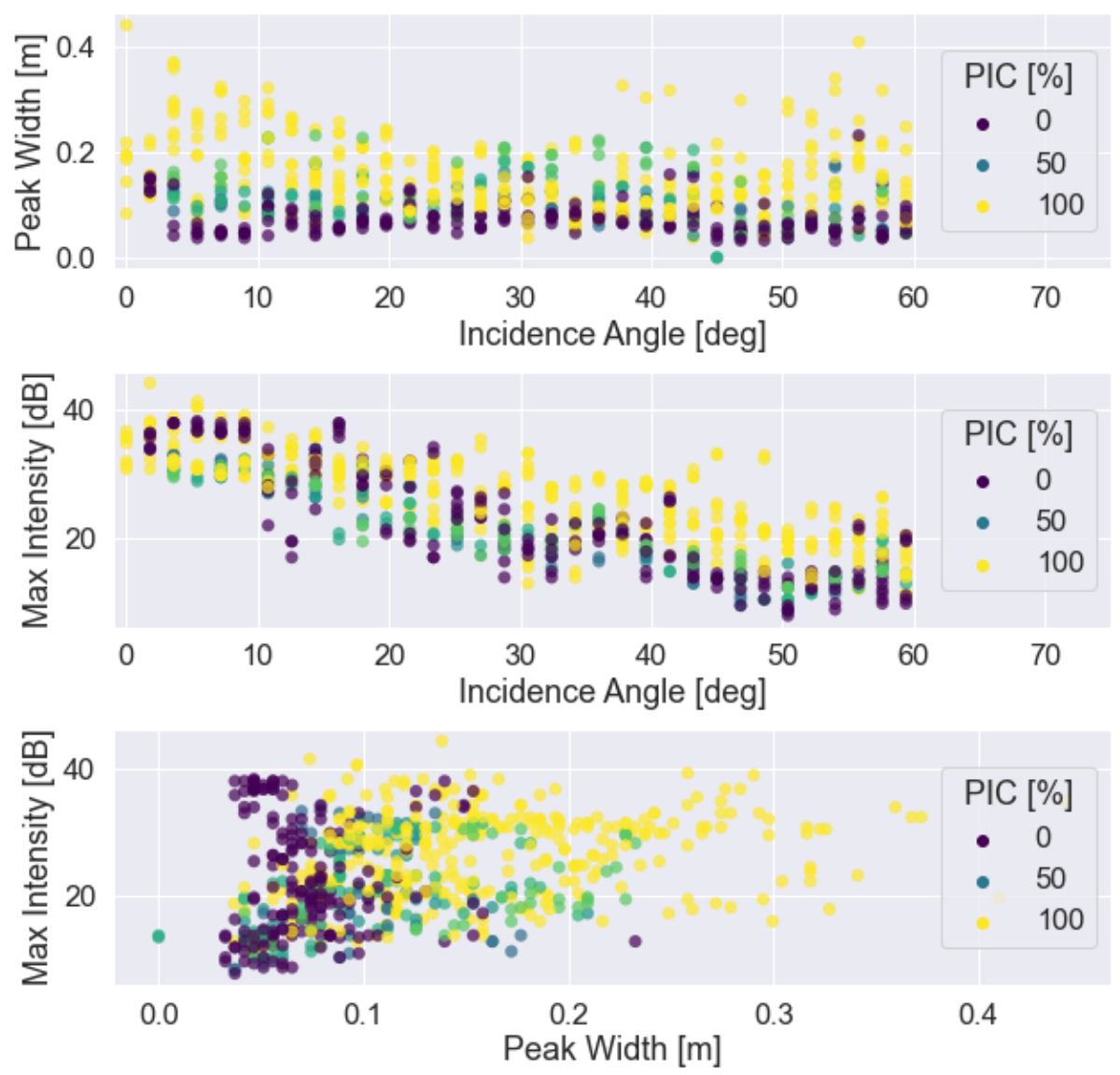

Figure 3-12: Acoustic features of sea-ice for field data collected in Saint-Fabien, QC. Peakwidth, incidence angle, and maximum intensity are plotted as 3D coordinates in three-view format. The color of each point indicates percent ice cover (PIC), where dark blue denotes free-surface, yellow denotes full ice cover, and green or purple colors indicate partial ice cover.

tections are made given individual acoustic pings. One benefit of analyzing the classifier performance given a single acoustic ping is that this approach makes no assumption regarding the MSIS control strategy. However, for increased classification performance, it is beneficial to consider an ensemble of neighboring acoustic pings. This is analogous to applications in computer vision: it is more challenging to classify an object based on the value of individual pixel rather than classifying an object based on neighboring pixel information like a convolution neural net based strategy. Therefore, to demonstrate the capability of 
using neighboring acoustic scanline information, sea-ice detection is also performed based on a moving window of the 10 most recent acoustic pings recorded, which is referred to as an acoustic swath.

Figure 3-13 shows the SSIM ice detection performance via inspection of the corresponding ROC curves. Note that ROC curves are reported separately for the two datasets. For each dataset, both the single-ping and the swath-of-pings detection schemes are shown. As expected, the swath of pings approach leads to greater classification performance for both datasets. The line of no-discrimination, which represents the performance of the random classifier, is included for reference.

\section{ROC Curves for Sea-ice Detection}

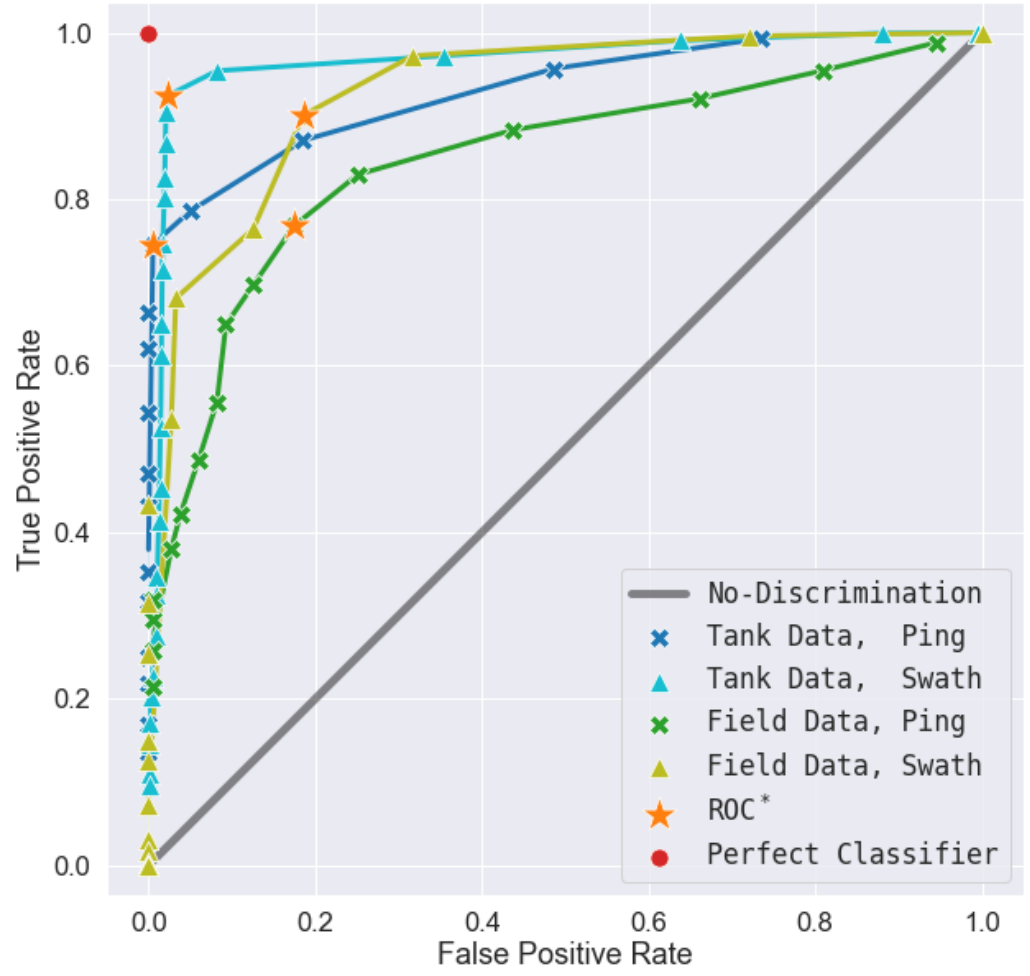

Figure 3-13: Receiver Operating Characteristic (ROC) curves for SSIM sea-ice detection, evaluated on laboratory and field datasets. Both laboratory and field datasets are evaluated with respect to single-ping and swath-of-pings classifiers. The optimal ROC curve points are indicated with orange stars, and the point of perfect classification and line of no-discrimination are included for reference.

The optimal point on the ROC curve depends on the relative cost of the two error 
conditions: False Positive (Type I error) or False Negative (Type II error). As a reminder, a False Positive signifies that the detector interprets free-surface conditions as sea-ice cover, and False Negative signifies that the detector interprets ice cover as free surface. The former is costly because the AUG may be forced to remain submerged longer than intended, leading to worsened navigation performance, and the latter is also costly because the AUG may attempt to perform a surfacing maneuver into ice cover. For simplicity, it is assumed that both errors are equally costly, and this determines that the optimal point on the ROC curve is the the point that is geometrically closest to perfect classifier position at $(0,1)$. The optimal ROC curve points are shown visually in Figure 3-13, and they are also displayed numerically in Table 3.5.

\begin{tabular}{|c|c|c|c|c|}
\cline { 2 - 5 } \multicolumn{1}{c|}{} & \multicolumn{2}{c|}{ Tank Data } & \multicolumn{2}{c|}{ Field Data } \\
\cline { 2 - 5 } & $\begin{array}{c}\text { FPR } \\
{[\%]}\end{array}$ & $\begin{array}{c}\text { TPR } \\
{[\%]}\end{array}$ & $\begin{array}{c}\text { FPR } \\
{[\%]}\end{array}$ & $\begin{array}{c}\text { TPR } \\
{[\%]}\end{array}$ \\
\hline Individual Ping & $\mathbf{0 . 5}$ & 74.6 & $\mathbf{1 7 . 5}$ & 76.8 \\
\hline Ensemble of Pings & 2.4 & $\mathbf{9 2 . 4}$ & 18.6 & $\mathbf{9 0 . 1}$ \\
\hline
\end{tabular}

Table 3.5: True Positive Rate (TPR) and False Positive Rate (FPR) for two sea-ice detection modes: detection based on individual MSIS pings and detection based on an ensemble of neighboring MSIS pings. TPR and FPR rates are given for both laboratory and field experiments, which are described in Section 3.6. The metrics listed correspond with the optimal ROC curve points that are indicated by orange stars in Figure 3-13.

As shown numerically in Table 3.5, and visually in Figure 3-13, the consideration of multiple ensembles at once can improve the performance of the sea-ice detection system. Note that the individual ping detector has a lower FPR than the ensemble of pings detector because both error conditions have been deemed as equally costly and the classifier is designed to minimize the geometric distance to the point of perfect classification. Thus, the marginal improvement in TPR outweighs the marginal regress in FPR. If errors with different relative cost are considered instead, the detector will be designed to favor the minimization of the more costly error.

Next, the impact that PIC has on classifier performance is considered. To do so, the classifier TPR is shown as a function of PIC for fixed values of FPR. By setting fixed values of FPR, it is possible to make sensible comparisons between the classifier performance on each dataset. Figure 3-14 displays TPR as a function of PIC for fixed values of FPR. As expected, the high values of PIC are consistently easier to perform successful sea-ice 
detection, as conveyed by the general positive linear trend in the plot. Interestingly, for the laboratory data-set, the classifier shows impressive classification given minimal PIC. The ability to detect sea-ice despite only partial ice coverage is especially valuable from an AUG survivability standpoint. The same data displayed in Figure 3-14 is shown in tabular format in Table 3.6.

\section{True Positive Rate for Laboratory Data and Field Data}

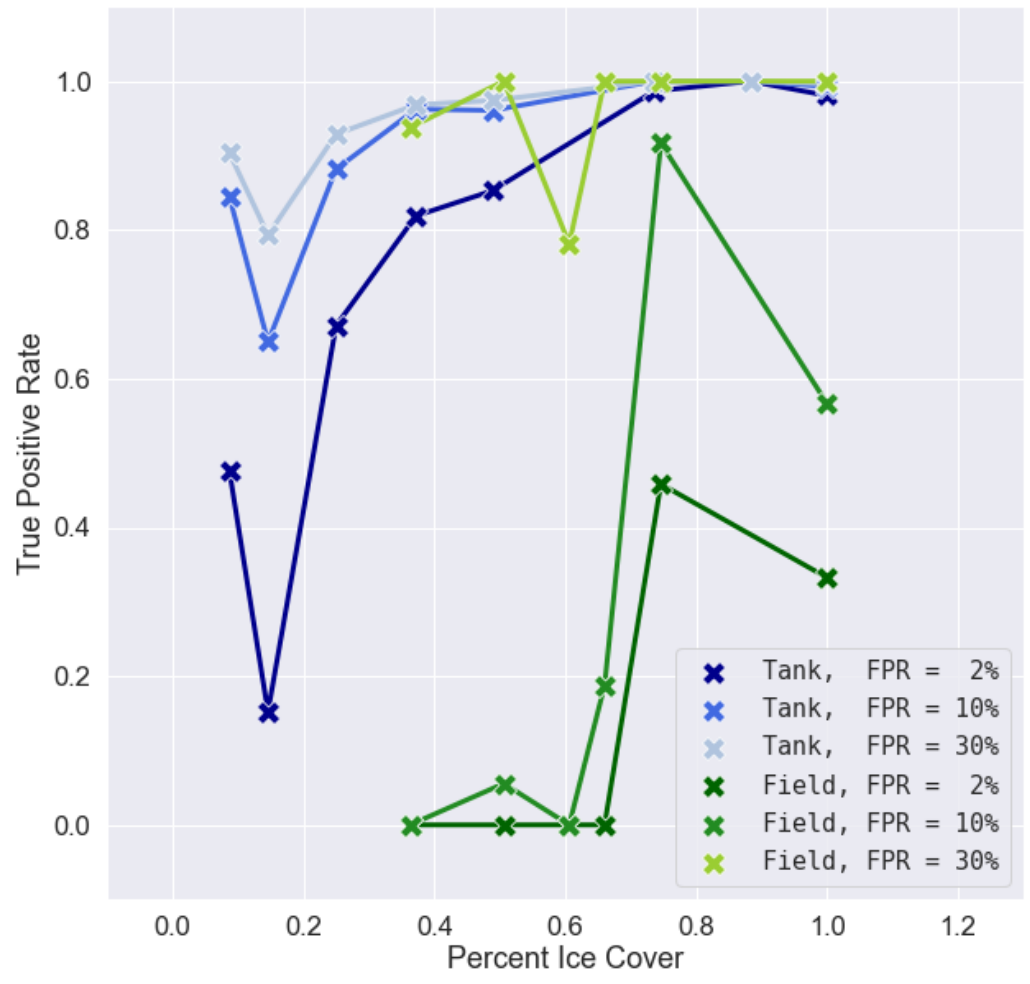

Figure 3-14: True Positive Rate (TPR) sea-ice detection performance as a function of Percent Ice Cover (PIC) for fixed levels of False Positive Rate (FPR). Both laboratory and field datasets are considered. High PIC conditions have better TPR performance than low PIC conditions, indicated by the general positive trend of the plot.

It is important to note that the hand-annotated labels for ice-presence in both the laboratory and field datasets have limited accuracy. Often, the transition from sea-ice cover to free surface was ambiguous. As a result, some instrument bearing angles where the MSIS ensonified the edge of sea-ice cover were mislabeled as free surface. Therefore, the classification performance presented in this chapter is hindered by the limited accuracy of 


\begin{tabular}{|c||c|c|c||c|c|c||c|c|c|}
\cline { 2 - 10 } \multicolumn{1}{c|}{} & \multicolumn{9}{c|}{ Woods Hole Test Tank Data } \\
\cline { 2 - 10 } \multicolumn{1}{c|}{} & \multicolumn{3}{c|}{ FPR $=2 \%$} & \multicolumn{3}{c|}{ FPR $=10 \%$} & \multicolumn{3}{c|}{ FPR $=30 \%$} \\
\hline PIC & TPC & CPC & TPR & TPC & CPC & TPR & TPC & CPC & TPR \\
{$[\%]$} & $n$ & $n$ & {$[\%]$} & $n$ & $n$ & {$[\%]$} & $n$ & $n$ & {$[\%]$} \\
\hline 8.7 & 545 & 1143 & $\mathbf{4 7 . 7}$ & 966 & 1143 & $\mathbf{8 4 . 5}$ & 1033 & 1143 & $\mathbf{9 0 . 4}$ \\
\hline 14.6 & 177 & 1164 & $\mathbf{1 5 . 2}$ & 756 & 1164 & $\mathbf{6 4 . 9}$ & 923 & 1164 & $\mathbf{7 9 . 3}$ \\
\hline 25.0 & 829 & 1235 & $\mathbf{6 7 . 1}$ & 1089 & 1235 & $\mathbf{8 8 . 2}$ & 1147 & 1235 & $\mathbf{9 2 . 9}$ \\
\hline 37.2 & 981 & 1198 & $\mathbf{8 1 . 9}$ & 1153 & 1198 & $\mathbf{9 6 . 2}$ & 1160 & 1198 & $\mathbf{9 6 . 8}$ \\
\hline 48.9 & 1020 & 1196 & $\mathbf{8 5 . 3}$ & 1149 & 1196 & $\mathbf{9 6 . 1}$ & 1165 & 1196 & $\mathbf{9 7 . 4}$ \\
\hline 73.6 & 1492 & 1512 & $\mathbf{9 8 . 7}$ & 1512 & 1512 & $\mathbf{1 0 0 . 0}$ & 1512 & 1512 & $\mathbf{1 0 0 . 0}$ \\
\hline 88.3 & 1197 & 1197 & $\mathbf{1 0 0 . 0}$ & 1197 & 1197 & $\mathbf{1 0 0 . 0}$ & 1197 & 1197 & $\mathbf{1 0 0 . 0}$ \\
\hline 100.0 & 10867 & 11082 & $\mathbf{9 8 . 1}$ & 11002 & 11082 & $\mathbf{9 9 . 3}$ & 11043 & 11082 & $\mathbf{9 9 . 6}$ \\
\hline
\end{tabular}

\begin{tabular}{|c||c|c|c||c|c|c||c|c|c|}
\cline { 2 - 10 } \multicolumn{1}{c|}{} & \multicolumn{9}{c|}{ Saint-Fabien Field Deployment Data } \\
\cline { 2 - 10 } \multicolumn{1}{c|}{} & \multicolumn{3}{c|}{ FPR $=2 \%$} & \multicolumn{3}{c|}{ FPR $=10 \%$} & \multicolumn{3}{c|}{ FPR $=30 \%$} \\
\hline PIC & TPC & CPC & TPR & TPC & CPC & TPR & TPC & CPC & TPR \\
{$[\%]$} & $n$ & $n$ & {$[\%]$} & $n$ & $n$ & {$[\%]$} & $n$ & $n$ & {$[\%]$} \\
\hline 36.3 & 0 & 32 & $\mathbf{0 . 0}$ & 0 & 32 & $\mathbf{0 . 0}$ & 30 & 32 & $\mathbf{9 3 . 8}$ \\
\hline 50.6 & 0 & 36 & $\mathbf{0 . 0}$ & 2 & 36 & $\mathbf{5 . 6}$ & 36 & 36 & $\mathbf{1 0 0 . 0}$ \\
\hline 60.6 & 0 & 32 & $\mathbf{0 . 0}$ & 0 & 32 & $\mathbf{0 . 0}$ & 25 & 32 & $\mathbf{7 8 . 1}$ \\
\hline 66.0 & 0 & 32 & $\mathbf{0 . 0}$ & 6 & 32 & $\mathbf{1 8 . 8}$ & 32 & 32 & $\mathbf{1 0 0 . 0}$ \\
\hline 74.6 & 22 & 48 & $\mathbf{4 5 . 8}$ & 44 & 48 & $\mathbf{9 1 . 7}$ & 48 & 48 & $\mathbf{1 0 0 . 0}$ \\
\hline 100.0 & 108 & 325 & $\mathbf{3 3 . 2}$ & 184 & 325 & $\mathbf{5 6 . 6}$ & 325 & 325 & $\mathbf{1 0 0 . 0}$ \\
\hline
\end{tabular}

Table 3.6: Analysis of how TPR performs on different PIC conditions, given three fixed values of FPR: $2 \%, 10 \%$, and $30 \%$. As expected, low PIC values are consistently more difficult to detect correctly compared to high PIC values. The number of points that fall into each category are given by the True Positive Count (TPC) and Condition Positive Count (CPC). Note that the TPR values shown in the table correspond with plotted points in Figure 3-14.

ground-truth surface information.

\subsection{Summary and Future Work}

This chapter reviews properties of sea-ice, develops an acoustics-based model that enables detection of sea-ice, and evaluates this feature-based classification approach on laboratory test data and field deployment data. The efforts of this chapter support the development of a SSIM module that performs onboard sensor interpretation and control of an MSIS sonar that is mounted in the nosecone of the Polarsentinel AUG. The SSIM module characterizes surface conditions based on three hierarchical attributes: sea-ice presence, sea-ice thickness, 
and sea-ice roughness. The predominant focus of this chapter was on the detection aspect of the SSIM module because sea-ice detection is particularly challenging in thin first-year sea-ice conditions.

Leveraging the sea-ice feature representation described in this chapter, an interesting future work item would be the determination of the sea-ice scattering function $S\left(\omega_{i}, \omega_{r}\right)$, as estimated with empirical data. As acoustic scattering is intimately connected with seaice structure, it may be interesting from a scientific perspective for a network of AUGs to persistently estimate the scattering function of sea-ice. In a similar vein, NASA utilizes persistence satellite observation to estimate the BRDF of Earth's surface [Schaaf, 2020]. In a slightly more obscure instance, researchers have defined the reflectance map of the human face, including the effects of sub-surface scattering, given a plethora of human face observations [Debevec et al., 2000].

In addition to sea-ice characterization, is is also important for the AUG to evaluate environmental state with regard to the sea-state, and in particular, wave magnitude and wave propagation direction. Using the same MSIS sonar, it is possible to employ power spectral analysis with a set of sequential observations to determine wavelength and propagation direction [Burgess et al., 2020]. Other related efforts of characterizing sea-state include the estimation of near-surface turbulence using an ADCP [Zippel and Thomson, 2016], and measuring wave-number using an airborne lidar system [Sutherland and Gascard, 2016].

Other than enabling acquisition of scientific data, control of the MSIS also impacts the AUG energy budget via two mechanisms: duty cycling of sensing and control systems in response to vehicle and environment state, and satellite communication during vehicle surfacing. As a result of using the hierarchical scheme for characterizing sea-ice as described in Section 3.4, the energy efficiency of satellite communication can be greatly improved because only a small subset of abstracted and compressed sea-ice information is required to be sent over the energetically expensive satellite link rather than the full raw data complement. More careful treatment of energy optimal AUG control policies is presented in Chapter 5. 
This page intentionally left blank. 


\section{Chapter 4}

\section{Multi-Factor Terrain-Aided}

\section{Navigation}

\subsection{Introduction}

Navigation is one of the unifying challenges that is at the heart of all mobile robotics development, a challenge that is critical for both vehicle survivability and scientific data collection. Although navigation is comprised of three core components, namely localization, path planning, and map building, this thesis chapter focuses primarily on the localization aspect of navigation. When operating in underwater environments, robots do not have access to the most ubiquitous navigation solution: the Global Positioning System (GPS). As a result, navigation is particularly challenging for AUVs operating in surface-denied regions such as the sea-ice covered Arctic since AUVs cannot rely on periodic GPS updates to constrain navigation error. Thus, AUVs must rely on alternative methods for navigation, which can be divided into three broad categories: inertial, acoustic transponder, and geophysical. A brief review of each of these categories is provided in the following paragraphs. Then, the remainder of this chapter describes and evaluates a proposed navigation solution that is designed for a sea-ice surveying AUG. More discussion on the path planning aspect of navigation is provided in Chapter 5 in the context of the Adaptive Velocity Control (AVC) algorithm, and more discussion of the map building aspect of navigation is discussed as a future work item in Section 4.5 in the context of a Simultaneous Localization and Mapping (SLAM) architecture. 
Inertial navigation systems (INS) generally use accelerometers or gyroscopes to observe the acceleration vector, which can then be twice integrated in time to yield a position update. Alternatively, for AUGs that pursue sawtooth trajectories, a combination of vehicle pitch and depth change over time can be used to estimate through-water velocity, which can then be integrated in time to estimate vehicle position [Claus and Bachmayer, 2015]. The strengths of INS navigation solutions are that they are self-contained and acoustically quiet. Being self-contained allows INS to be range-independent and have minimal external infrastructure requirements, while being acoustically quiet allows for minimal impact on marine mammals and the possibility for stealth missions. The primary weakness of INS navigation solutions is that position error grows unbounded due to the fact that position is derived by integration acceleration or velocity observations over time. With the exception of military-grade INS systems, which are generally expensive, energetically costly, and restricted by the International Traffic in Arms Regulations (ITAR), INS navigation solutions lead to significant error in position estimates a result of this unbounded growth. If INS is reinforced with a DVL instrument for estimating water column currents, navigation performance can be significantly improved [Kinsey and Whitcomb, 2004, Medagoda et al., 2015]. Additionally, the incorporation of model-based filters that account for nonlinear vehicle dynamics models can further improve navigation performance of INS [Arnold and Medagoda, 2018].

Acoustic transponder navigation solutions leverage Time of Flight (TOF) measurements to estimate range and bearing information between an AUV and acoustic transponders, where the transponders are positioned at a known locations. Two common configurations for acoustic transponder navigation include Long Baseline (LBL) and Ultra-short Baseline (USBL). LBL systems involve placing a set of acoustic beacons throughout the region of operation so that the AUV can triangulate its position using range estimates from multiple beacons. Conventionally, an AUV emits an interrogation signal that instigates a response from the beacons, and then the two-way travel time (TWTT) of the signal is used to estimate range. However, an alternative LBL implementation involves the AUV acting as a passive listener and using one-way travel time (OWTT) to deduce range, but this involves highly accurate clock synchronization between the AUV and the beacons. LBL systems can be highly accurate and reliable for AUV navigation [Whitcomb et al., 1999, Yoerger et al., 2007, Jakuba et al., 2008] and have demonstrated success during AUG sea-ice survey in the 
Arctic at mesoscale ranges [Webster et al., 2015]. However, LBL systems do not extend well to basin-scale operations or regions with complex bathymetry due to the infrastructure cost of placing beacons throughout the region of operation and the need to maintain line-of-sight between the AUV and the beacons. On the other hand, USBL systems involve mounting an acoustic transponder array on a surface support vessel so that TOF and signal phase can be used to estimate range and bearing relative to the AUV. Conventionally, an AUV emits an acoustic signal so that the surface vessel can deduce the position of the AUV. However, recent efforts include an inverted USBL design where the AUV acts as a passive listener that can deduce its position relative to the support vessel [Rypkema et al., 2017]. USBL systems can also be highly accurate for AUV navigation [Rigby et al., 2006, Jakuba et al., 2015, Morgado et al., 2011], but similar to LBL systems, they suffer from limited range. Although USBL systems allow for more operational flexibility than LBL systems because the surface vessel can dynamically update its position in response to the AUV location, USBL systems do not extend well to sea-ice survey operations because the presence of sea-ice precludes the use of surface vessels.

Geophysical methods use fixed external environment information paired with onboard sensing for environment feature extraction to perform navigation. Environment information that can be used for AUV navigation include bathymetric [Claus and Bachmayer, 2015, Anonsen and Hallingstad, 2006, Teixeira et al., 2015, Salavasidis et al., 2019, Lucido et al., 1996], magnetic [Pasnani et al., 2018, Armstrong et al., 2010, Armstrong et al., 2009], and gravimetric [Kinsey et al., 2008, Kinsey et al., 2013]. Due to its relatively large spatial variance and perceptibility with standard sensor suites, seafloor bathymetry is the most commonly used field variable for geophysical navigation. The class of algorithms that leverage terrain information to improve vehicle navigation is referred to as Terrainaided Navigation (TAN). Bathymetric features for TAN can be extracted using acoustic sensors [Claus and Bachmayer, 2015, Teixeira et al., 2015] or optical sensors [Eustice et al., 2005, Hover et al., 2012]. The benefits of geophysical navigation are that minimal support infrastructure is required since environment features are extracted via onboard sensing, and that localizing with respect to fixed environmental features leads to bounded error as opposed to the unbounded error growth experienced by an INS system. That said, geophysical methods have their limitations as well: navigation accuracy is dependent on the accuracy and resolution of the ground-truth map, the existence of features in the environment, and 
the ability of the AUV to make accurate observations of said features. In sections of the high Arctic, the best resolution available for ground-truth digital elevation maps (DEM) is $200 \mathrm{~m}$ [Jakobsson et al., 2012]. For operating in unknown or resolution-limited environments such as the Arctic, Simultaneous Localization and Mapping (SLAM) techniques can be pursued [Barkby et al., 2011, Barkby et al., 2009, Mallios et al., 2014, Mallios et al., 2016, Johnson-Roberson et al., 2010].

In general, AUV navigation typically involves a combination of the aforementioned approaches. For example, it is common to combine inertial and acoustic transponder methods [Kepper et al., 2018, Morgado et al., 2013, Hegrenaes et al., 2009, Van Uffelen et al., 2013], or inertial and geophysical methods [Donovan, 2012, Medagoda et al., 2011]. For more extensive reviews of AUV navigation methods, the reader is referred to a set of excellent survey papers [Paull et al., 2014, Kinsey et al., 2006, Leonard and Bahr, 2016, Stutters et al., 2008, Melo and Matos, 2017]. For GPS-denied sea-ice survey in the Arctic, AUGs can utilize DVL-reinforced inertial navigation in combination with terrain-based geophysical navigation to determine AUG position with bounded error. In this way

\subsection{Doppler Velocity Log Odometry}

The navigation system for the sea-ice surveying AUG consists of an inertial component and a geophysical component. The inertial component of the navigation system utilizes a DVL instrument to actively sense water column currents to improve odometry; hence, this component is referred to as DVL-Odometry (DVL-Odo). DVL-Odo is composed of two subroutines: a routine for estimating AUG through-water velocity and a routine for estimating ocean currents. The goal of DVL-Odo is to determine an accurate estimate of vehicle overground velocity $\mathbf{v}_{o g}$. Once an estimate for $\mathbf{v}_{o g}$ is obtained, it can be integrated forward in time to estimate the evolution of AUG position. As a result of this time-integration, even small disturbances in the estimate for $\mathbf{v}_{\text {og }}$ will lead to unbounded error growth in vehicle position estimates. To reduce this navigation error, the AUG either receives a GPS while at the surface, or the AUG localizes itself with respect to the seafloor via a TAN process.

Over-ground velocity $\mathbf{v}_{\text {og }}$ can be written as the sum of through-water $\mathbf{v}_{t w}$ and ocean current velocity $\mathbf{v}_{o c}$. Note that $\mathbf{v}_{o g}$ and $\mathbf{v}_{o c}$ are given in the absolute world frame, and $\mathbf{v}_{t w}$ 
is given in the relative vehicle frame.

$$
\mathbf{v}_{o g}=\mathbf{v}_{t w}+\mathbf{v}_{o c}
$$

Subsection 4.2.1 describes a method for estimating $\mathbf{v}_{t w}$ via dead-reckoning (DR), and Subsection 4.2.2 describes a method for estimating $\mathbf{v}_{o c}$ via velocity shear propagation (VSP). Together, they enable estimation of over-ground velocity throughout the water column via Equation (4.1), and then this over-ground velocity is integrated in time to yield position estimates for the AUG. Of course, when the AUG is within sensing range of the seafloor, $\mathbf{v}_{\text {og }}$ is given directly from the bottom-track velocity recorded by the DVL. However, this estimate is only available in a limited portion of the water column. By utilizing an estimation process based on DR and VSP, the AUG can estimate $\mathbf{v}_{\text {og }}$ throughout the water column, even when the seafloor is not observable. Additionally, estimating $\mathbf{v}_{o c}$ allows for the AUG to take advantage of energy optimal control policies that are presented in Chapter 5.

\subsubsection{Dead Reckoning}

Due to the sawtooth nature of the AUG trajectory, the through-water velocity can be measured via a combination of depth, pitch and compass sensors, as shown in [Claus and Bachmayer, 2015]. This approach for measuring through-water velocity is particularly advantageous because depth, pitch, and compass sensors are relatively low power and accurate

to better than $1 \%$ of dynamic range. Let $\mathbf{v}_{t w}=\left(\begin{array}{lll}u_{t w} & v_{t w} & w_{t w}\end{array}\right) \mathrm{T}$ be the components of the through-water velocity vector, with $u$ pointing to the East, $v$ pointing to the North, and $w$ pointing to vertically downward. The vertical velocity can be measured as a change in depth over a change in time.

$$
w_{t w}=\frac{\Delta z}{\Delta t}
$$

Then, using the glide angle as a sum of the AUG pitch $\phi$ and angle of attack $\alpha, \xi=\phi+\alpha$, and heading measurement $\boldsymbol{\psi}$, the through-water velocity in the horizontal plane can be written as: 


$$
\begin{aligned}
& u_{t w}=\frac{w_{t w}}{\tan \xi} \sin \psi \\
& v_{t w}=\frac{w_{t w}}{\tan \xi} \cos \psi
\end{aligned}
$$

Putting the three equations together, the through-water velocity estimate is given as a function of measurements from the pressure sensor, pitch sensor, and compass. This estimate is referred to as the dead-reckoned (DR) estimate of through-water velocity.

$$
\mathbf{v}_{t w}=\left(\begin{array}{ccc}
\frac{\Delta z}{\tan \xi} \sin \psi & \frac{\Delta z}{\tan \xi} \cos \psi & \frac{\Delta z}{\Delta t}
\end{array}\right) \mathrm{T}
$$

\subsubsection{Ocean Current Estimation}

After obtaining an estimate for through-water velocity, the AUG must determine the relationship between the relative vehicle frame and the absolute world frame to estimate ocean current velocities. The AUG can do this via two primary mechanisms: using surface drift velocity obtained from successive GPS measurements or using bottom-track DVL velocities when the seafloor is within range of the DVL. For the surface drift case, $\mathbf{v}_{\text {og }}$ is measured by successive GPS fixes at known times when $\mathbf{v}_{t w}=0$ as the AUG is floating in a Lagrangian manner, which leads to $\mathbf{v}_{o c}=\mathbf{v}_{o g}$. For the DVL bottom-lock velocities, the DVL instrument measures $\mathbf{v}_{\text {og }}$ by measuring the Doppler shift of acoustic ping returns from stationary seafloor surfaces. Then, ocean currents can be estimated by subtracting the through-water

velocity: $\mathbf{v}_{o c}=\mathbf{v}_{o g}-\mathbf{v}_{t w}$. Both mechanisms for establishing a reference to the world-frame are infrequently available during an AUG mission, making direct observation of absolute velocity sparse. Therefore, successive accumulation of velocity shear is required to propagate absolute velocity estimates when a world-frame reference is not directly observable [Visbeck, 2002, Todd et al., 2017].

The velocity shear propagation (VSP) method uses the difference between through-water velocity measurements to measure a velocity shear, where a velocity shear encodes the change in ocean current velocity across a specified change in depth. Specifically, a velocity shear is computed as the difference between the DR through-water velocity $\mathbf{v}_{t w, D R}$ and the DVL water-track through-water velocity $\mathbf{v}_{t w, D V L}$ at the same instance in time. This is possible because the DVL water-track velocity measurements are recorded at some distance 
$\Delta z$ away from the transducer head. By taking the difference of the through-water measurements at the same instance in time, the over-ground velocity terms cancel out, leaving behind the velocity shear term $\Delta \mathbf{v}_{o c}$.

$$
\begin{gathered}
\mathbf{v}_{t w, D R}=\mathbf{v}_{o g}-\mathbf{v}_{o c}(z) \\
\mathbf{v}_{t w, D V L}=\mathbf{v}_{o g}-\mathbf{v}_{o c}(z+\Delta z) \\
\mathbf{v}_{t w, D R}-\mathbf{v}_{t w, D V L}=\mathbf{v}_{o c}(z+\Delta z)-\mathbf{v}_{o c}(z)=\Delta \mathbf{v}_{o c}
\end{gathered}
$$

These velocity shear terms $\Delta \mathbf{v}_{o c}$ can be incrementally added together via forward propagation to estimate water column currents in a relative frame. Once an absolute velocity reference becomes available via GPS surface drift or DVL bottom-track, the absolute reference is back-propagated through the previous velocity shear measurements to provide water column velocity estimates in the absolute frame. Together, the forward and backward propagation routines comprise the VSP method of estimating ocean currents.

Although the VSP update equations are relatively simple to write down, performing VSP in practice is like taking a walk through Relativity, a lithograph print by M.C. Escher where multiple gravity vectors are depicted in a room full of orthogonal staircases; the AUG records through-water velocity estimates in a continuously evolving relative frame, which can make the deduction of the absolute reference frame disorienting. To help establish a concrete understanding of the VSP method in action, Figure 4-1 showcases a schematic example of how the AUG can back-out water column estimates $\mathbf{v}_{o c}$ from relative velocity measurements $\mathbf{v}_{t w}$ and an absolute velocity reference $\mathbf{v}_{o g}$ by performing backward propagation and forward propagation.

Once through-water velocity is estimated with DR and ocean currents are estimated with VSP, over-ground velocity can be integrated forward in time to estimate the position evolution of the AUG. Consequently, improving inertial estimates for AUG position also leads to performance improvement of the geophysical navigation method because the initial guess for AUG position is closer to the true AUG position, effectively reducing the search space of the geophysical method. 


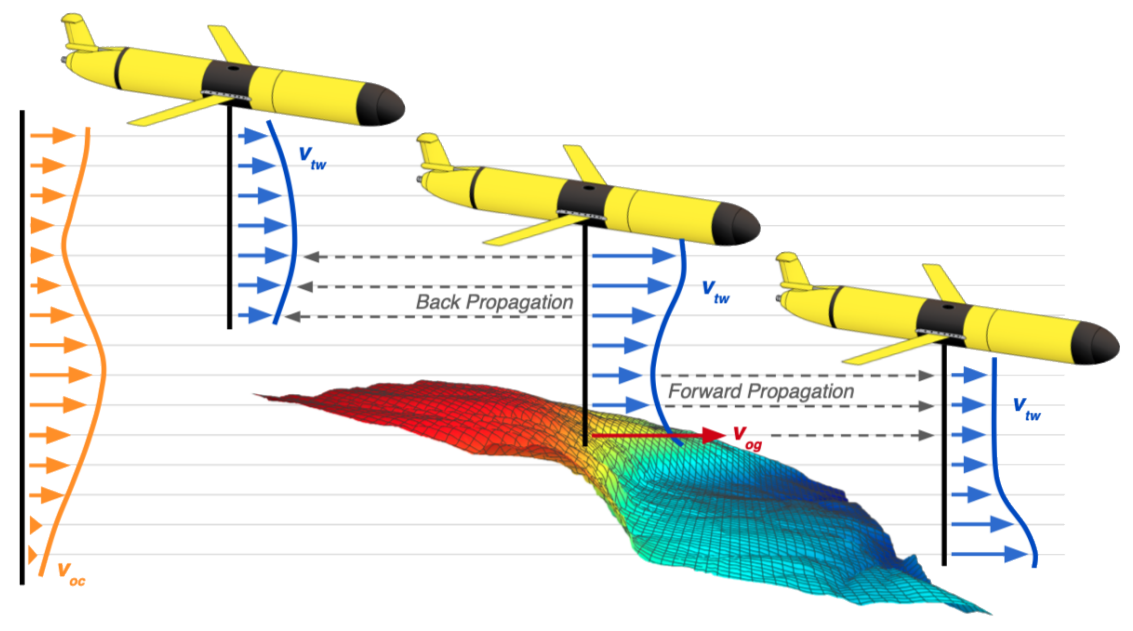

Figure 4-1: Schematic diagram of the Velocity Shear Propagation (VSP) method being used to estimate water column currents. The VSP method uses forward propagation and back propagation to estimate ocean currents throughout the water column.

\subsection{Multi-Factor Terrain-Aided Navigation}

The geophysical component of AUG navigation system entails a Terrain-Aided Navigation (TAN) algorithm that considers multiple characteristics of the seafloor, referred to as MultiFactor Terrain-Aided Navigation (MF-TAN). MF-TAN uses range estimates from the four beams of the DVL instrument to compute three geometric factors of the seafloor: depth, slope, and orientation. Then, these observed factors are used to localize the AUG with respect to ground-truth bathymetry. Provided that the seafloor is observable, the MF-TAN navigation method has bounded navigation error, which contrasts with DVL-Odo which experiences unbounded navigation error. The fact that MF-TAN has bounded navigation error is particularly important for Arctic sea-ice survey missions where the AUG is submerged for significant lengths of time. Note that MF-TAN can be viewed as a superset of the DVL-Odo method because DVL-Odo provides an initial pose estimate to the MF-TAN before localization with respect to the ground-truth bathymetry can be performed. Additionally, when seafloor features are unobservable, the AUG relies solely on the DVL-Odo algorithm to determine its position. Thus, this two-pronged navigation solution is wellsuited for AUGs that may spend significant portions of the missions without the ability to observe the seafloor due to their glide-path trajectory through the water column.

Conventional TAN methods only consider seafloor depth when performing localization 
with respect to the ground truth map. Figure 4-2 motivates the use of additional seafloor factors by displaying the Digital Elevation Map (DEM), Digital Slope Map (DSM), Digital Orientation Map (DOM) for the Kolumbo Volcano underwater crater. As shown in the figure, each factor contains features that are independent of each other, which effectively increases the density of features available to the AUG to perform localization.

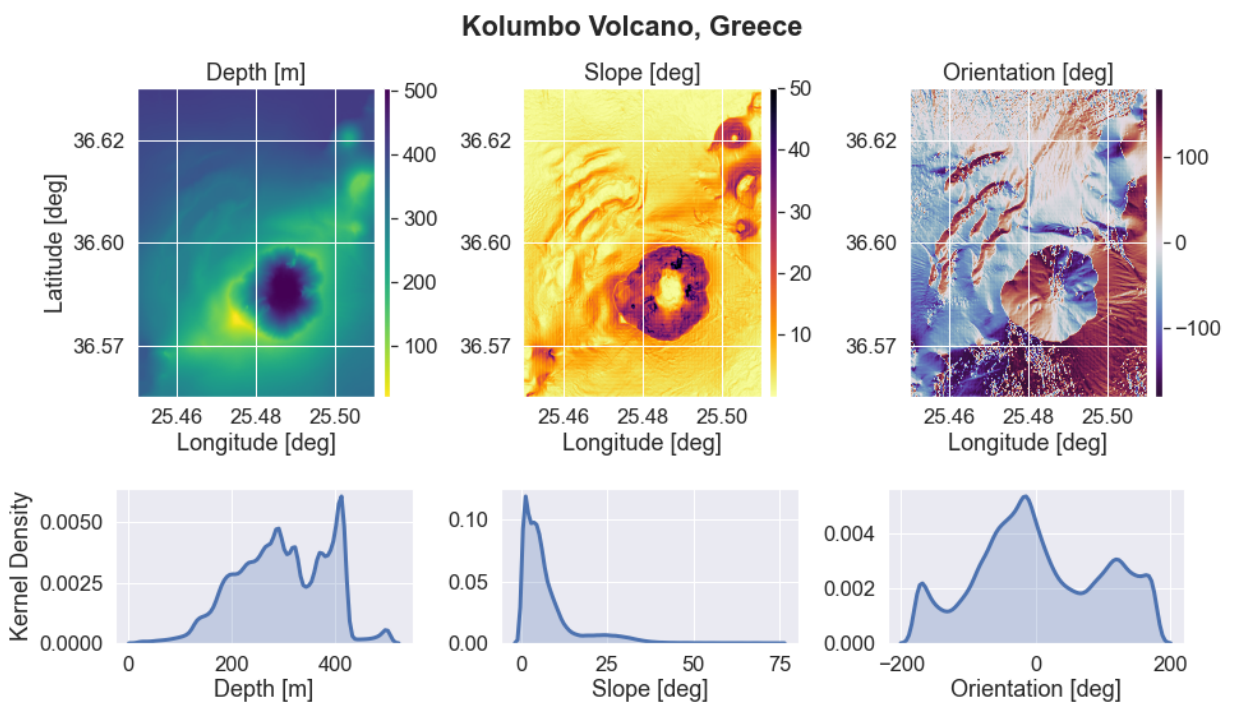

Figure 4-2: Three factors of seafloor for the Kolumbo Volcano underwater crater: depth, slope, and orientation. Together, these three factors comprise the ground-truth map available to the AUG when performing navigation via the MF-TAN algorithm. A kernel density plot is provided for each factor to convey the relative uniqueness of a particular location in the environment.

\subsubsection{Multi-Factor Extraction}

Three factors of seafloor are used to perform MF-TAN: depth, slope, and orientation. The AUG measures these factors by using range observations from the DVL instrument, where the four beams of the DVL instrument span a cross-sectional area of the seafloor below the AUG. Once DVL range estimates are collected, the MF-TAN method fits a plane to the points in 3D space using a least squares formulation, which allows for extraction of depth, slope, and orientation. Figure 4-3 displays a schematic diagram of seafloor factor extraction using the DVL instrument. Effectively, MF-TAN exploits the Janus configuration of the DVL beams in order to estimate seafloor slope and orientation, wheres conventional TAN formulations only consider seafloor depth. 


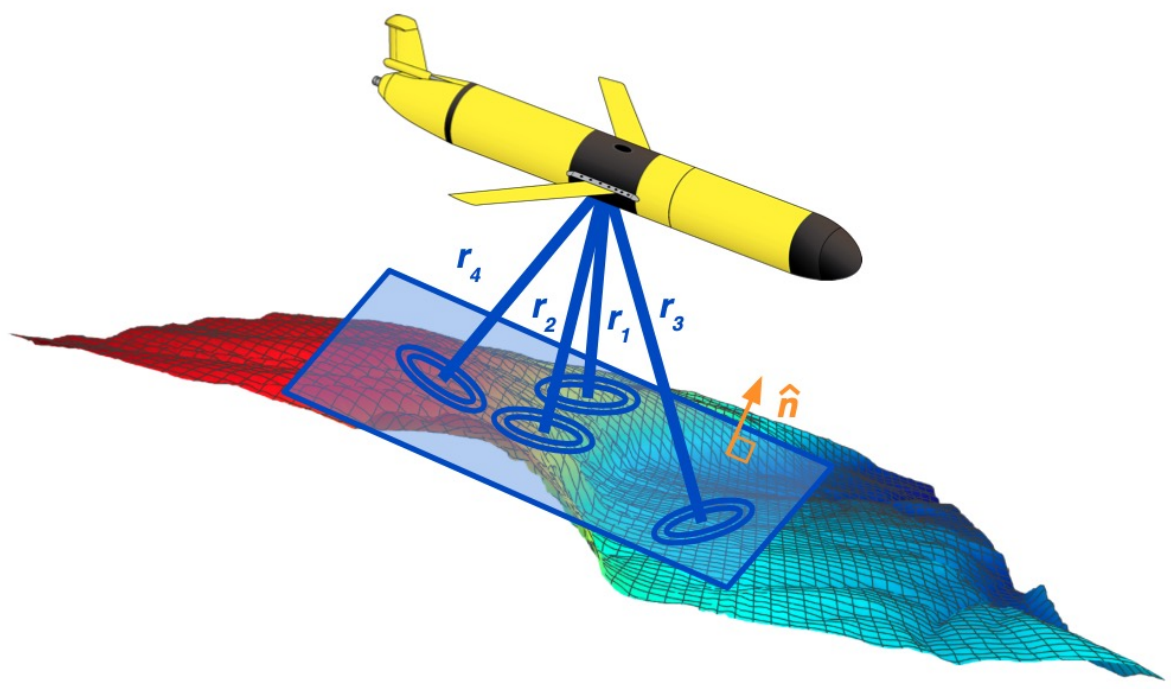

Figure 4-3: Schematic diagram of seafloor factor extraction using the DVL instrument. To extract seafloor factors, a plane is fit to a set of bottom-contact points in 3D space. Then, seafloor depth, slope, and orientation can be readily extracted and used by the MF-TAN algorithm.

To match the resolution of the ground-truth bathymetric map, several successive DVL observations are considered in aggregate when extracting seafloor factors. Using multiple DVL observations for factor extraction prevents the AUG from oversampling the environment compared to the ground-truth map. For example, a ground-truth bathymetry map may be gridded at $10 \mathrm{~m}$ resolution, but when the AUG is flying at an altitude of $5 \mathrm{~m}$, the DVL beams cover an area of approximately $6 \mathrm{~m}$ in terms of spatial extent. In this situation, the AUG would be vulnerable to detecting high frequency features in the environment that would not be found when consulting the ground-truth map. In this situation, the DVL observations can be treated as the continuous signal the ground-truth map as the discrete sampling of the continuous signal. Then, the Nyquist sampling theorem states that the discrete signal sample rate must be at least twice the maximum frequency contained by the continuous signal in order to prevent aliasing. Since the sample frequency of the ground-truth map is fixed, the DVL observations are instead spatially averaged to twice the length of the bathymetric map griding to obey the Nyquist sampling criterion. In addition to matching the resolution of the ground-truth map, the consideration of multiple DVL observations also improves the stability of the seafloor factor extraction process. 


\subsubsection{Particle Filter}

Once the AUG extracts factors from the seafloor, the factors can be used in combination with other AUG state variables to perform localization with respect to the ground-truth bathymetry map. To solve this filtering problem, a particle filter (PF) formulation is employed. The PF, also known as Sequential Monte Carlo (SMC), uses random sampling to estimate posterior distributions of dynamical systems given partial and noisy observations without making assumptions about the state-space of the model or the state distributions. Although PF do not extend well to high dimensional systems, they perform well for largescale and nonlinear systems, and as a result, they have been employed in some recent TANbased AUV navigation efforts [Paull et al., 2014, Claus and Bachmayer, 2015, Donovan, 2012].

Leveraging the formulation described in [Donovan, 2012], let $\mathbf{x}_{t}$ represent the state of the AUG at time $t, \mathbf{y}_{t}$ represents the observation acquired at time $t, \mathbf{Y}_{t}$ represents the full history of observations up to time $t$. Under the Markov assumption, state transition and observation processes only depend on the previous state and the current state respectively. For AUG navigation, the posterior distribution of particular interest is denoted by $p\left(\mathbf{x}_{t} \mid \mathbf{Y}_{t}\right)$, which is the probability of the current state given the observation history of the AUG. Then, the evolution of the posterior distribution equation can be written as a two-step recursion with update and prediction steps:

$$
\begin{aligned}
p\left(\mathbf{x}_{t} \mid \mathbf{Y}_{t}\right) & =\frac{p\left(\mathbf{y}_{t} \mid \mathbf{x}_{t}\right) p\left(\mathbf{x}_{t} \mid \mathbf{Y}_{t-1}\right)}{\int p\left(\mathbf{y}_{t} \mid \mathbf{x}_{t}^{\prime}\right) p\left(\mathbf{x}_{t}^{\prime} \mid \mathbf{Y}_{t-1}\right) d \mathbf{x}_{t}^{\prime}} \\
p\left(\mathbf{x}_{t+1} \mid \mathbf{Y}_{t}\right) & =\int p\left(\mathbf{x}_{t+1} \mid \mathbf{x}_{t}\right) p\left(\mathbf{x}_{t} \mid \mathbf{Y}_{t}\right) d \mathbf{x}_{t}
\end{aligned}
$$

Since the distributions shown in Equation (4.6) and Equation (4.7) can be intractable to compute analytically, they are approximated by a set of weighted particles where the weight of each particle represents the likelihood that the particle was drawn from the desired distribution. Although the PF produces an approximate solution for the posterior distribution, the accuracy of the PF can be improved by increasing the number of particles. However, increasing the number of particles also increases computational time required to perform filtering. Thus, the number of particles included in the PF requires careful consideration 
regarding the trade-off between accuracy and computational time.

Although the PF can produce accurate approximations of the posterior distribution, two common failure modes of the PF must be addressed: sample impoverishment and particle collapse. Sample impoverishment occurs when the weight distribution amongst the particles becomes uneven and a large number of particles have negligible weight. In this situation, the expressivity of the PF is reduced and the PF may no longer be able to accurately estimate the posterior distribution. To overcome this failure mode, particle resampling techniques are employed where the particles with negligible weight are replaced by new particles that are placed in the vicinity of higher weight particles. This resampling approach exacerbates the next failure mode: particle collapse, which occurs when the majority of the particles collect in a narrow section of the state space. Particle collapse diminishes the ability of the $\mathrm{PF}$ to react to future measurements, and thus, the PF is more likely to get trapped in local minima. To overcome particle collapse, process noise is added to each particle in a process known as jittering to artificially spread out the particles in the state space. Together, the base $\mathrm{PF}$ algorithm with resampling and jittering processes included is known as Sequential Importance Resampling (SIR). Then, if resampling is performed at every time-step and strong jittering is utilized to prevent particle collapse due to frequent resampling, the filter becomes a Jittered Bootstrap Particle Filter (JBPF). The JBPF has been shown to provide robust navigation estimates in response to sparse observations and relatively featureless terrain [Claus and Bachmayer, 2015, Houts et al., 2013, Bar-Shalom et al., 2004].

To enable robust navigation of the AUG, the MF-TAN algorithm utilizes the JBPF. The novelty of the MF-TAN algorithm pertains to how particles are resampled and weights are readjusted in response to multiple seafloor factors observed by the AUG and the navigation drift reported by DVL-Odo. By including multiple seafloor factors in the filtering process, MF-TAN is able to discard a larger number of suboptimal particles compared to conventional TAN methods that only consider seafloor depth, and as a result, MF-TAN reaches an accurate navigation solution more readily. In the case of sparse seafloor observations, which is common for AUGs that pursue sawtooth trajectories in the water column, MF-TAN is more likely to converge to the correct navigation solution because it utilizes more environmental constraints to bound the viable positions of the AUG. That said, it is important to recall that the accuracy of MF-TAN is dependent on the accuracy of the ground-truth bathymetric map and the accuracy of the extracted seafloor features. 


\subsection{Results}

Our methods for ocean current estimation with VSP, navigation with DVL-Odo, and navigation with MF-TAN are evaluated using AUG sea-trial data from a November 2019 deployment within an active submarine volcano in the Southern Aegean Sea. For this set of experiments, a Slocum AUG was used as a reconnaissance platform to provide initial surveys of the Kolumbo caldera crater to inform subsequent ROV mission planning. During these missions the AUG relied on its default DR process, which included a static current correction that calculated a temporal and depth-averaged water column current based on discrepancy between the GPS and DR localization estimates during its prior dive. This DR with depth-averaged current correction (DR-DACC) does not directly observe ocean current velocities and is unable to account for temporally dynamic currents or biasing caused by variability in the AUG's depth band.

\subsubsection{Doppler Velocity Log Odometry}

Results of the VSP ocean current estimation process and DVL-Odo navigation are shown in Figure 4-4. Although access to independent water column currents observations are not available for comparison, the strong performance of DVL-Odo serves as evidence that the water column current estimates are accurate. Accuracy of DVL-Odo is confirmed by comparing DVL-Odo navigation to GPS position updates when the AUG is at the surface. It is important to note that DVL-Odo is not performing Terrain-Aided Navigation (TAN) with known bathymetry maps in this figure. Instead, DVL-Odo estimates the water column currents with the VSP method according to Section 4.2.2, and these water column currents are used to improve estimates of over-ground velocity $\mathbf{v}_{\text {og }}$.

In Dive A, the AUG travels almost due north for approximately $6000 \mathrm{~m}$, while staying submerged underwater for approximately $2.5 \mathrm{~h}$. When the AUG surfaces, DVL-Odo indicates a localization error equal to $4.4 \%$ of distance traveled whereas DR-DACC indicates a localization error of $11.9 \%$ of distance traveled. The AUG observes a strong southward ocean current, approaching $0.5 \mathrm{~m} \mathrm{~s}^{-1}$ in magnitude, for the majority of the water column, along with a small northward counter-current near the sea surface. In Dive B, the AUG identifies a south-eastward current in the upper $40 \mathrm{~m}$ of the water column and a northwestward current between $40 \mathrm{~m}$ and $80 \mathrm{~m}$. Dive B includes 2 GPS surface updates during 



Figure 4-4: Offline ocean current sensing for a selection of AUG missions during the November 2019 deployment in the Southern Aegean Sea. Each row of the figure corresponds to a particular mission. The AUG starts each mission at the origin of the local mean coordinates (LMC) coordinate system. The decreased localization error provided by DVL-Odo compared to DR-DACC serves as evidence that the water column currents are being estimated accurately.

the mission. DVL-Odo indicates a localization error of $16.9 \pm 12.2 \%$ of distance traveled while DR-DACC indicates a localization error of $51.9 \pm 14.4 \%$ of distance traveled. In Dive $\mathrm{C}$, the AUG identifies a low magnitude westward current throughout the water column, as well as a higher magnitude westward current at approximately $100 \mathrm{~m}$ depth. Dive C includes 10 GPS surface updates during the mission. DVL-Odo indicates a localization error of $23.1 \pm 13.5 \%$ of distance traveled while DR-DACC indicates a localization error of $97.7 \pm 52.6 \%$ of distance traveled.

\subsubsection{Multi-Factor Terrain-Aided Navigation}

This section discusses the results MF-TAN, including: factor extraction, JBPF resampling, and AUG navigation. The section concludes with navigation results for the DR-DACC, 
DVL-Odo, and MF-TAN algorithms applied to the AUG missions during the November 2019 research deployment in the Southern Aegean Sea. First, Figure 4-5 shows the result of seafloor factor extraction for Dive A of the Southern Aegean Sea missions. In Dive A, the AUG transited out of the Kolumbo crater and then transited approximately $6000 \mathrm{~m}$ North of the crater. During the transit, the seafloor increases in depth, decreases in slope, and dynamically changes in orientation. Note that for small slope angles, the measured orientation factor of the seafloor is arbitrary, which is indicated by the sporadic values for the orientation factor during the last leg of Dive A. As a result, the orientation factor is not included as a constraint in the JBPF filtering process when slope values are below a critical threshold.

Since the ground-truth bathymetry is gridded at $10 \mathrm{~m}$, a point cloud of DVL bottomtrack points are collected until the point cloud covers $20 \mathrm{~m}$ in lateral distance before seafloor factors are extracted via a least squares procedure, in accordance to the Nyquist sampling theorem as discussed in Subsection 3.7.1. Additionally, DVL bottom-track points recorded during bottom reflections were not included in the point cloud for feature extraction due to time lag identified between the pitch sensor and the DVL instrument. In future implementations, this time lag can be accounted for as part of an automated calibration process.

Next, the seafloor factors deduced from DVL observations are used to localize the AUG with respect to a ground-truth bathymetry map using the JBPF filtering method. Figure 4-6 portrays two instances of the MF-TAN localizing the AUG position with respect to the ground-truth map, one within the Kolumbo crater and one outside the Kolumbo crater. The subplots in the figure highlight the portions of the environment that were consistent with the seafloor factors measured by the AUG.

With access to the observed seafloor factors and DVL-Odo navigation uncertainty, the MF-TAN algorithm performs particle resampling and particle propagation. To do so, the JBPF computes particle weights based on the uncertainty ellipse provided by DVL-Odo and the seafloor factors measured by the AUG. A set of tolerance parameters were included for the seafloor factors such that the acceptable tolerance for a particular factor was designed to match the relative observability of the factor. As such, the depth factor has the most strict tolerance and the slope factor has the most lenient tolerance. To improve computational efficiency, the most restrictive constraints are considered first. In this implementation, a simple model of position uncertainty growth was utilized where uncertainty was assumed 

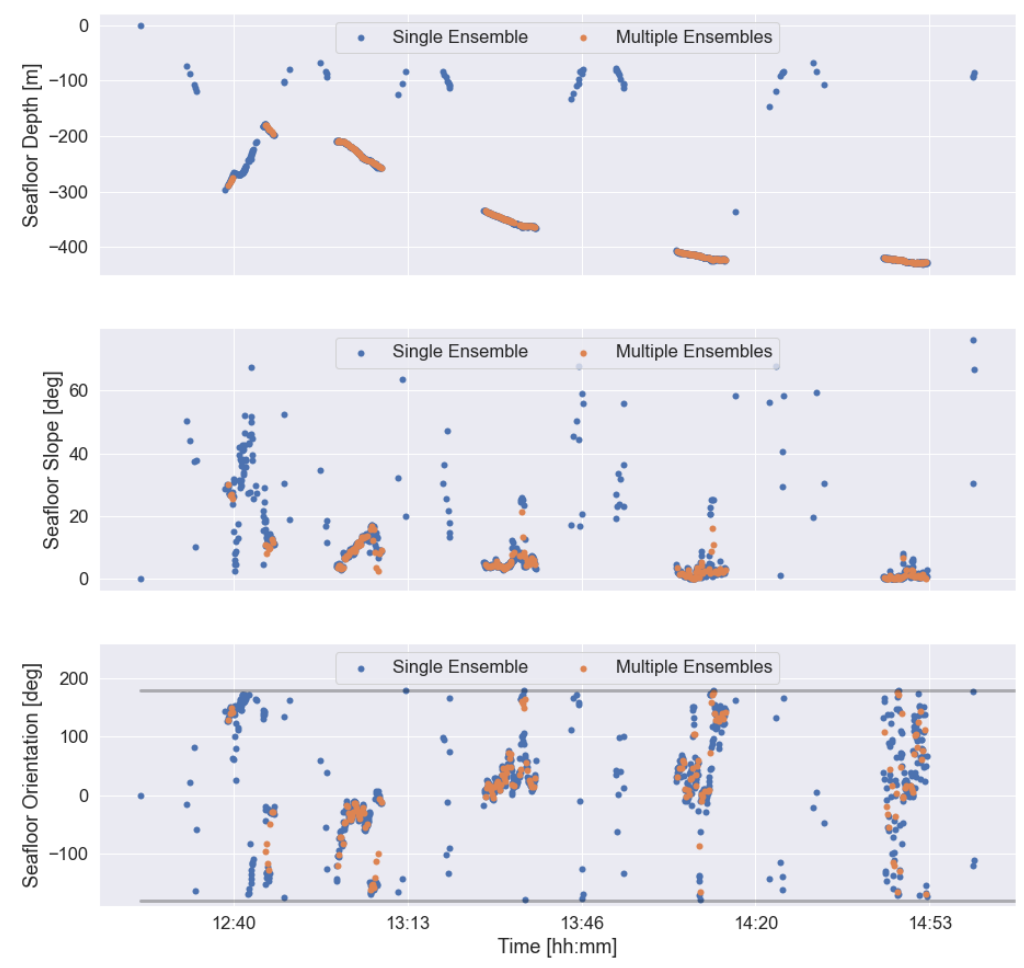

Figure 4-5: Bathymetric factor extraction for Dive A of the November 2019 deployment in the Southern Aegean Sea. The factor extraction shows how stability can be improved by considering successive DVL ensembles rather than just considering a single ensemble at each time-step. Note that the depth factors present at approximately $100 \mathrm{~m}$ water depth are caused by stratification in the water column.

to expand radially outward at a rate of $20 \%$ of distance traveled, which is slightly greater than the expected error growth of DVL-Odo. Although this is a crude approximation for position uncertainty, it allows for a proof-of-concept for the MF-TAN algorithm. In future implementations, it may be beneficial to pursue a model-based approach such as the Extended Kalman Filter (EKF). After particle weights have been assigned, particles below a critical weight threshold, new particles are added in the near vicinity of the highest weight particles, and jittering is added to the particle position. In this implementation, a maximum of 100 particles were considered at a time, but the precise strategy for setting the optimal number of particles for the MF-TAN algorithm requires further investigation. Once particles have been resampled, particles are propagated forward in time according to 
A)



B)

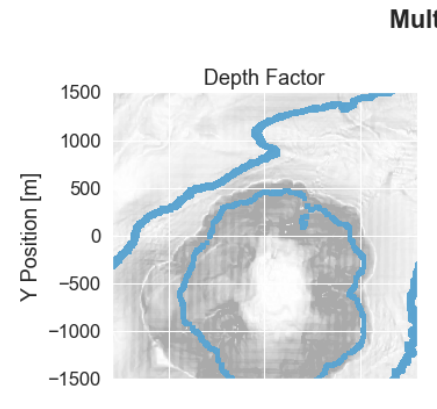

Multi-Factor Terrain-Aided Navigation
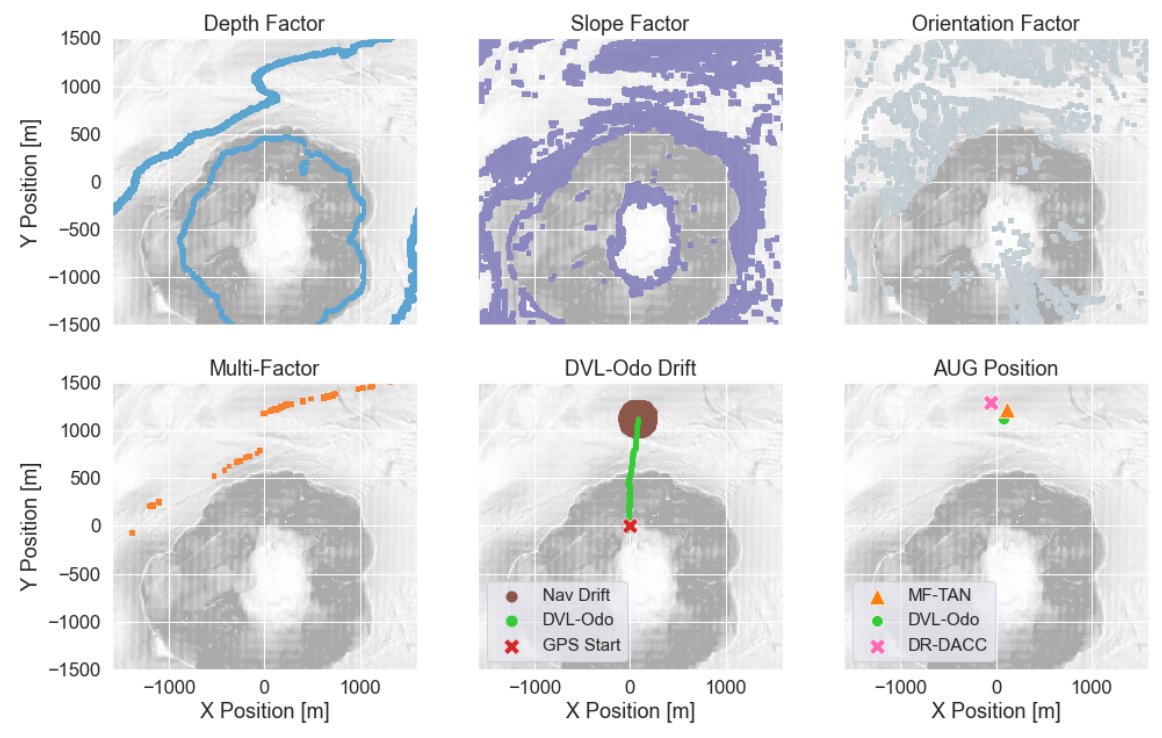

Figure 4-6: MF-TAN localization using three factors of the seafloor. The figure contains two subplots: Subplot A shows when the AUG performs localization within the Kolumbo crater, and Subplot B shows when the AUG performs localization outside of the crater. Each subplot highlights the three factor constraints in isolation, as well as the intersection of the constraints. When the factors are considered in combination with DVL-Odo position drift, the AUG is able to localize itself with respect to the ground-truth bathymetry.

the $\mathbf{v}_{\text {og }}$ estimated by DVL-Odo.

The main benefit of the MF-TAN algorithm compared to conventional TAN algorithms 


\begin{tabular}{|c|c|c||c|c||c|c||c|}
\hline Dive & $\begin{array}{c}\text { TAN Fix } \\
n\end{array}$ & $\begin{array}{c}\text { Distance } \\
{[\mathrm{m}]}\end{array}$ & $\begin{array}{c}\text { DF } \tilde{\sigma} \\
{\left[100 \mathrm{~m}^{2}\right]}\end{array}$ & $\begin{array}{c}\text { DF } \bar{\sigma} \\
{\left[100 \mathrm{~m}^{2}\right]}\end{array}$ & $\begin{array}{c}\text { MF } \tilde{\sigma} \\
{\left[100 \mathrm{~m}^{2}\right]}\end{array}$ & $\begin{array}{c}\text { MF } \bar{\sigma} \\
{\left[100 \mathrm{~m}^{2}\right]}\end{array}$ & $\begin{array}{c}\text { Red. } \\
{[\%]}\end{array}$ \\
\hline A & 42 & 6063 & 12.5 & $99.5 \pm 306$ & 9.5 & $95.1 \pm 304$ & $\mathbf{1 5 . 4} \pm \mathbf{1 3 . 8}$ \\
\hline D & 9 & 433 & 5.0 & $5.2 \pm 2.5$ & 2.0 & $2.8 \pm 2.0$ & $\mathbf{3 8 . 6} \pm \mathbf{3 1 . 9}$ \\
\hline E & 59 & 647 & 14.0 & $14.2 \pm 4.9$ & 7.0 & $7.7 \pm 5.5$ & $\mathbf{4 6 . 2} \pm \mathbf{2 9 . 8}$ \\
\hline F & 24 & 810 & 10.5 & $12.6 \pm 6.3$ & 3.0 & $4.0 \pm 3.5$ & $\mathbf{6 1 . 1} \pm \mathbf{3 1 . 8}$ \\
\hline G & 58 & 421 & 9.0 & $8.6 \pm 2.6$ & 4.0 & $4.2 \pm 2.1$ & $\mathbf{4 7 . 4} \pm \mathbf{2 7 . 6}$ \\
\hline \hline Agg. & 192 & 8374 & 10.0 & $30.5 \pm 147.7$ & 5.0 & $25.1 \pm 146.8$ & $\mathbf{4 1 . 3} \pm \mathbf{3 1 . 9}$ \\
\hline
\end{tabular}

Table 4.1: Comparison of Depth-Factor (DF) and Multi-Factor (MF) seafloor constraints during TAN fixes for the November 2019 deployment in the Southern Aegean Sea. DF and MF constraints are given as areas, corresponding to the portion of the ground-truth bathymetric map that is consistent with the factor(s) observed by the AUG. For each dive, median area $(\tilde{\sigma})$ and mean area $(\tilde{\sigma})$ are reported. The final column shows the percent reduction in area that occurs when using MF instead of DF. The final row of the table reports the aggregate of the five dives.

is that MF-TAN leverages two additional seafloor factors, namely seafloor slope and seafloor orientation, to narrow the set of viable states where the AUG can be positioned. By narrowing the set of viable states, MF-TAN can more readily avoid local minima solutions and more readily recover a valid terrain fix after a sustained period without seafloor observations. Table 4.1 shows how the inclusion of slope and orientation seafloor factors were able to reduce the size of the viable state-space for the AUG. The table reports the size of the viable state space in units of area for both the conventional Depth Factor (DF) approach and the novel Multi-Factor (MF) approach. Across 5 dives and almost 200 TAN fixes, the MF allows for a $41.3 \pm 31.9 \%$ reduction in the size of the viable state space compared to DF. Note that Dive A experiences a particularly high mean and standard deviation as a result of the relatively feature-limited bathymetry during the end of the dive and the relatively long dive sections with no seafloor measurements. It is important to note that the size of the viable state-space is sensitive to the tolerance factors chosen for each depth factor. For example, if the tolerance parameters are overly strict, the viable area reported by the JBPF will be exceedingly small, which will negatively impact AUG navigation by contributing to particle collapse.

After MF-TAN continually localizes the AUG throughout the mission when seafloor measurements are available, the resulting navigation trajectory can be compared to GPS updates retrieved when the AUG surfaces. Figure 4-7 shows the result of three factor extraction from the environment and the resulting trajectory devised by the MF-TAN navigation 
algorithm for Dive F of the Southern Aegean Sea deployment. Then, Figure 4-8 shows the navigation performance for four dives during the deployment in the Southern Aegean Sea that contain bottom-track data. Finally, Table 4.2 compares navigation performance numerically between the MF-TAN, DVL-Odo, and DR-DACC navigation methods.

\section{Bathymetric Factor Extraction for MF-TAN}
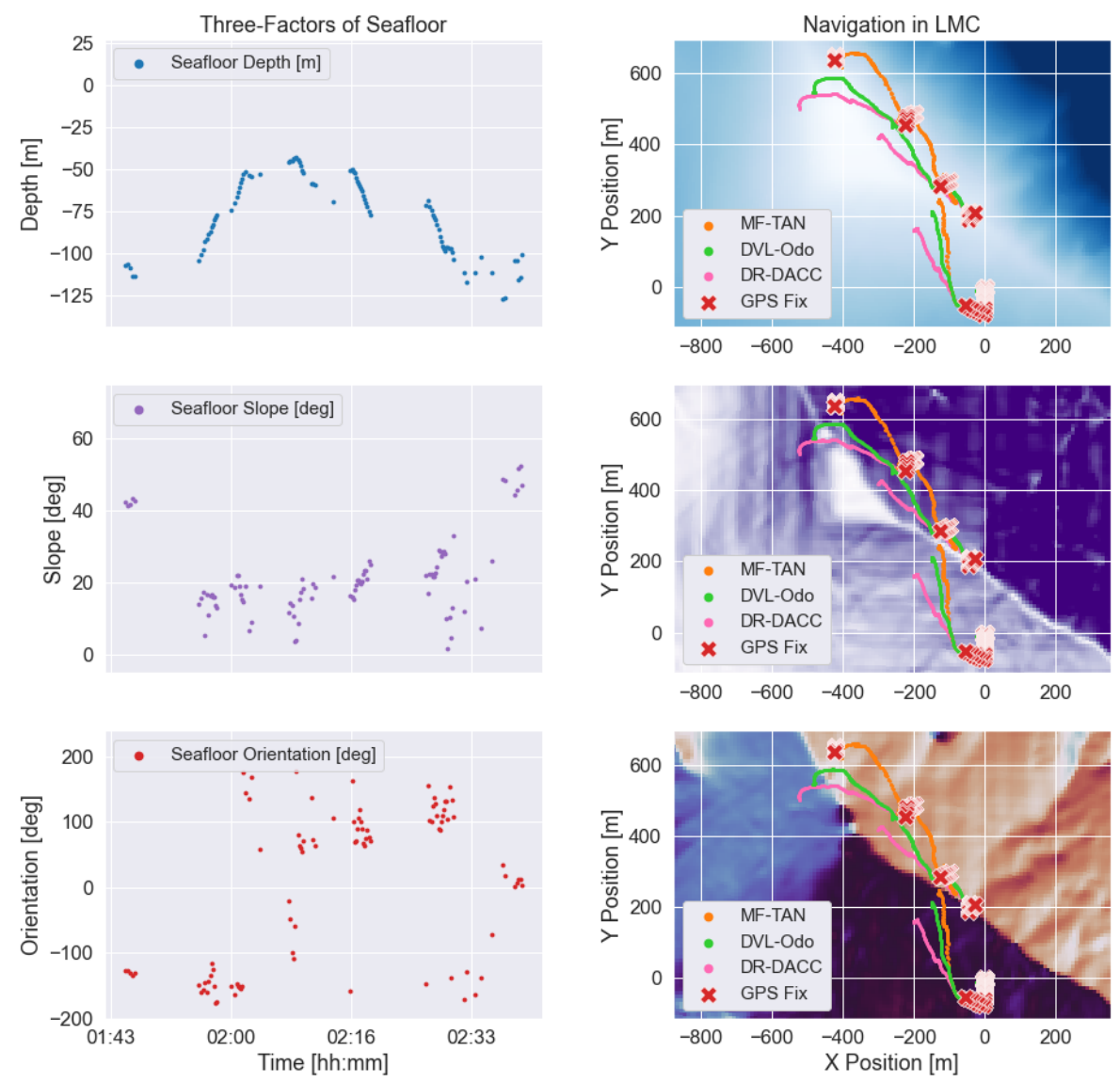

Figure 4-7: Bathymetric factor extraction and MF-TAN navigation for Dive F of the November 2019 deployment in the Southern Aegean Sea. The depth, slope, and orientation factors correspond with the first, second, and third rows of the figure subplots. The MF-TAN method leverages three factors of seafloor bathymetry to significantly improve navigation performance over the DVL-Odo and DR-DACC methods.

In Dive F, the dive which had the largest reduction in viable state-space when utilizing MF instead of DF 4.1, MF-TAN is able to take advantage of all three seafloor factors to improve AUG navigation. In this dive, the AUG crosses over the Southwestern rim of the 


\begin{tabular}{|c|c|c||c|c|c||c|c|}
\hline Dive & $\begin{array}{c}\text { Legs } \\
n\end{array}$ & $\begin{array}{c}\text { Distance } \\
{[\mathrm{m}]}\end{array}$ & $\begin{array}{c}\text { DR-DACC } \\
{[\%]}\end{array}$ & $\begin{array}{c}\text { DVL-Odo } \\
{[\%]}\end{array}$ & $\begin{array}{c}\text { MF-TAN } \\
{[\%]}\end{array}$ & $\begin{array}{c}\text { MF-TAN } \tilde{\epsilon} \\
{[\mathrm{m}]}\end{array}$ & $\begin{array}{c}\text { MF-TAN } \bar{\epsilon} \\
{[\mathrm{m}]}\end{array}$ \\
\hline A & 1 & 6063 & 11.9 & 6.8 & $\mathbf{1 . 5}$ & 93.6 & 93.6 \\
\hline D & 1 & 433 & 38.9 & 28.4 & $\mathbf{1 2 . 1}$ & 52.3 & 52.3 \\
\hline E & 4 & 647 & 88.7 & 44.6 & $\mathbf{3 4 . 6}$ & 55.2 & $56.0 \pm 26.4$ \\
\hline F & 4 & 810 & 64.8 & 40.4 & $\mathbf{2 1 . 3}$ & 32.9 & $43.1 \pm 28.2$ \\
\hline G & 3 & 421 & 33.2 & 32.4 & $\mathbf{1 3 . 4}$ & 14.2 & $18.8 \pm 10.9$ \\
\hline \hline Agg. & 13 & 8374 & 25.4 & 15.4 & $\mathbf{7 . 1}$ & $\mathbf{3 6 . 9}$ & $\mathbf{4 6 . 0} \pm \mathbf{2 9 . 5}$ \\
\hline
\end{tabular}

Table 4.2: Performance comparison between three methods of navigation: Dead Reckoning with Depth-averaged Current Correction (DR-DACC), DVL Odometry (DVL-Odo), and Multi-Factor Terrain-Aided Navigation (MF-TAN). Navigation error is given as a percentage of distance traveled for the three methods, and the median $(\tilde{\epsilon})$ and mean $(\bar{\epsilon})$ navigation errors in $[\mathrm{m}]$ are given for the MF-TAN method. Standard deviations are also listed for dives with multiple legs. The final row of the table shows the aggregate navigation performance across the five AUG missions. The AUG missions are from the November 2019 research deployment in the Southern Aegean Sea.

Kolumbo Volcano underwater crater. When traversing over the rim of the caldera, the slope and orientation factors are particularly informative: the slope factor momentarily records relatively low values as the AUG passes over the apex of the crater rim, and the orientation factor records a dramatic shift when AUG traverses from one side of the crater to the other. Together, the exploitation of the three seafloor factors allow the MF-TAN to achieve localization error that is approximately twice as low as DVL-Odo which does not have any terrain-aided correction.

Table 4.2 showcases the navigation results for the MF-TAN, DVL-Odo, and DR-DACC methods, as applied to the AUG missions from the November 2019 research deployment in the Southern Aegean Sea. Note that only AUG dives that contained bottom-track data were included in the table, because the MF-TAN would not be applicable for comparison otherwise. Navigation error is compared as a percent of distance traveled. However, unlike the DR-DACC and DVL-Odo methods, the MF-TAN method does not experience unbounded growth in navigation error. Thus, for MF-TAN, navigation error is also listed in $\mathrm{m}$ as a median and mean for the five dives during the deployment.

Interestingly, the MF-TAN algorithm achieves its lowest navigation error in terms of distance traveled in Dive A, which is the dive with the least amount of seafloor observations. This strong navigation performance for Dive A may be due to the already strong performance by the DVL-Odo algorithm, and the strong performance of the DVL-Odo al- 


\section{Dive A}

Multi-Factor Terrain-Aided Navigation

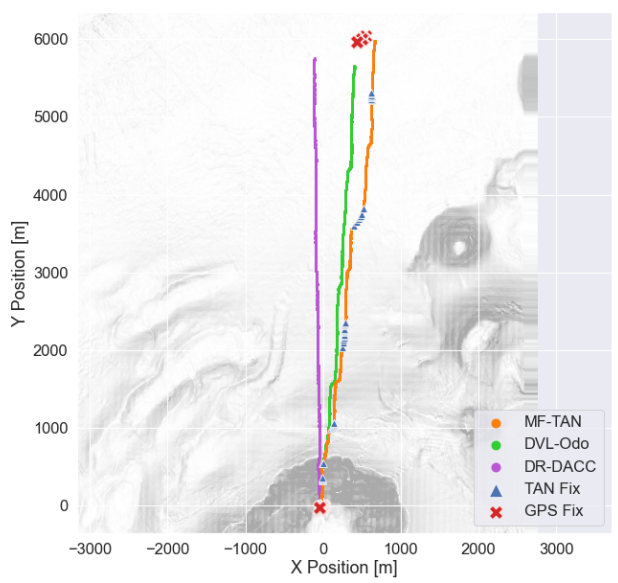

Dive $\mathrm{F}$

Multi-Factor Terrain-Aided Navigation

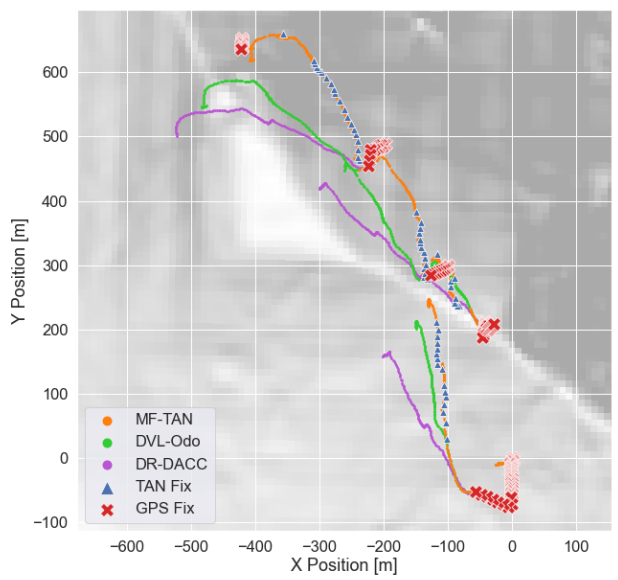

Dive $\mathrm{E}$

Multi-Factor Terrain-Aided Navigation

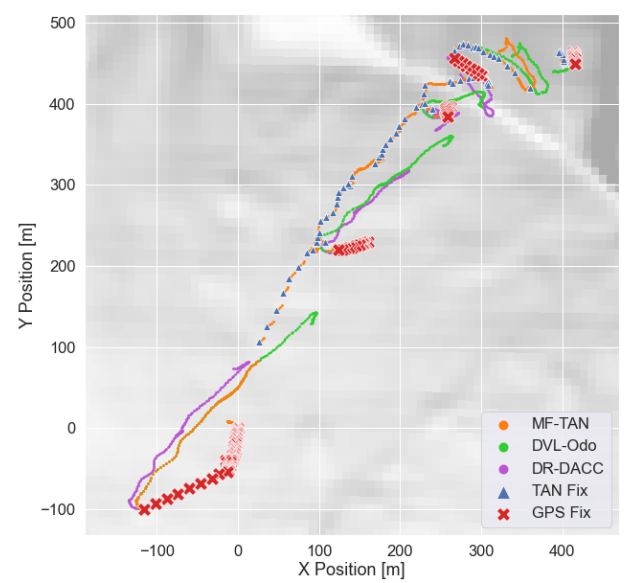

Dive $G$

Multi-Factor Terrain-Aided Navigation

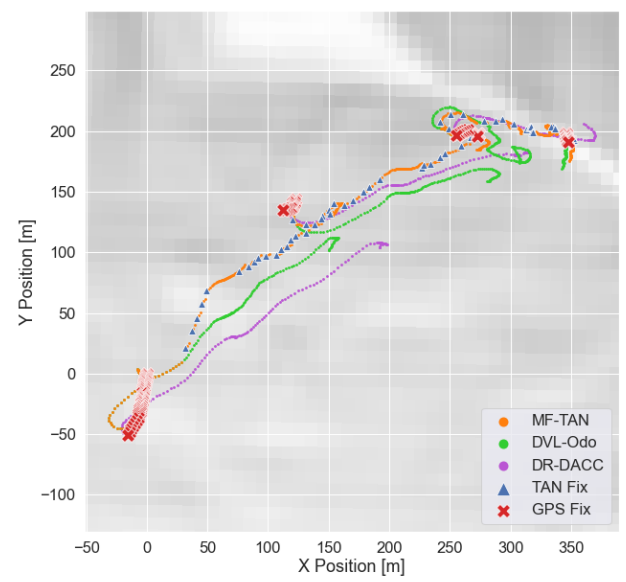

Figure 4-8: Multi-Factor Terrain-Aided Navigation (MF-TAN) for four dives during the November 2019 research deployment in the Southern Aegean Sea. MF-TAN is compared against DVL-Odo and DR-DACC, and both TAN fixes and GPS fixes are displayed. The MF-TAN navigation method consistently achieves the lowest navigation error compared to the other methods.

gorithm may be due to the simple flight path of the dive. This observation provides two valuable insights: (1) the performance of the MF-TAN algorithm is intimately connected to the performance of the DVL-Odo method, and (2) the navigation performance of the AUG is intimately connected to the chosen path for the AUG. The second insight, which is referred to as active localization, is expanded further as a future work item in Chapter 6.

In aggregate, the MF-TAN achieves a navigation error of $7.1 \%$ of distance traveled, 
which is approximately twice as low as DVL-Odo, and approximately four times as low as DR-DACC. Additionally, for all 13 legs across the 5 AUG dives, the MF-TAN algorithm achieves a median navigation error of $36.9 \mathrm{~m}$, and a mean navigation error of $46.0 \pm 29.5 \mathrm{~m}$. In conclusion, the minimal navigation error realized by MF-TAN makes it the ideal candidate for AUG sea-ice survey in the Arctic when the vehicle is denied access to the sea-surface for extended durations of time.

\subsection{Summary and Future Work}

This chapter presents an AUG navigation solution that combines inertial methods and geophysical methods. The DVL-Odo method leverages a DVL instrument to estimate water column currents in real-time to improve odometry of the inertial system. Then, MF-TAN builds upon DVL-Odo by considering multiple factors of the seafloor to perform localization with respect to a ground-truth map of the underlying bathymetry. Both DVL-Odo and MF-TAN are evaluated with respect to AUG missions during a November 2019 research deployment in the Southern Aegean Sea. During these dives, the MF-TAN navigation method consistently estimates vehicle position to within $100 \mathrm{~m}$ of the true AUG position, regardless of the distance traveled by the AUG during the dive.

The combination of inertial and geophysical methods for navigation is particularly helpful for remote robotic agents that are unable to rely on external infrastructure for navigation assistance. In an interesting parallel to the underwater domain, terrain-aided navigation methods have recently been deployed by NASA's Jet Propulsion Laboratory (JPL) in the context of the Entry Descent and Landing (EDL) pipeline for the Mars 2020 Perseverance Rover [NASA, 2020]. For the Mars 2020 mission, optical-based TAN is used to identify hazards in the environment and navigate the spacecraft to a safe landing site. Previous Mars expeditions required more risk-averse mission planning for EDL because the decision making of the landing system was fairly primitive. However, now that hazard risk is actively assessed in the EDL pipeline via an optical TAN process, JPL was able to confidently select a landing site in the near vicinity of dangerous terrain, which in turn allows the Perseverance Rover to pursue especially interesting science objectives. Ambitious mission planning in response to improved navigation is echoed in the underwater domain as well: by improving the navigation capabilities of AUGs in surface denied situations, mission concepts such 
as basin-scale sea-ice survey are enabled.

Future work for AUG navigation in the context of basin-scale sea-ice survey include the incorporation of more environmental field variables in the MF-TAN algorithm. For example, the magnetic anomaly in the Arctic could be used to supplement the three seafloor factors presented in this thesis to further refine navigation performance. Additionally, SLAM approaches to AUG navigation would be particularly useful in high-Arctic latitudes where ground-truth bathymetry is only available at a resolution of $200 \mathrm{~m}$. By actively building a high resolution map the seafloor, the AUG may be capable of precise navigation independent of the ground-truth map resolution. 
This page intentionally left blank. 


\section{Chapter 5}

\section{Energy-Optimal Control Policies}

\section{$5.1 \quad$ Introduction}

The intelligent allocation of onboard resources is critical for the success of AUG sea-ice survey operations in the Arctic. By actively limiting energy consumption while still making progress towards the science objective of the mission, the AUG is able to extend mission duration and range, increase the amount of science data that can be collected, and reduce the probability of catastrophic failure caused by total exhaustion of the onboard energy supply. Thus, pursuing AUG behaviors that enact intelligent allocation of onboard resources is universally beneficial for the four design requirements mentioned at the beginning of Chapter 2: scientific data collection, long-range travel, unattended operation, and survival.

In this chapter, the notion of energy efficiency is formalized by defining the transport cost function, and then a set of optimized vehicle behaviors that minimize the transport cost function is derived. The aforementioned optimized vehicle behaviors include methods for adaptive propulsion control, vertical path planning via the selection of the optimal AUG depth band, and active sensing control via duty cycling of acoustic sensors.

\subsection{Objective Function: Minimize Transport Cost}

Several objectives must be considered simultaneously when planning AUG missions. For example, objectives pertaining to range, endurance, localization, science, safety, communication, and rendezvous must be considered. Each of these objectives must be satisfied via the utilization of one common resource: the energy carried onboard the AUG. Therefore, 
efficient allocation of energy is universally beneficial for improving AUG missions.

When developing an AUG mission plan, the notion of resource management can be defined as one of several objectives that is considered in the context of a multi-objective optimization problem. This can be expressed as:

$$
\begin{aligned}
& \min _{\mathbf{u}}\left[f_{1}(\mathbf{u}), f_{2}(\mathbf{u}), \cdots, f_{k}(\mathbf{u})\right] \\
& \text { s.t. } \mathbf{u} \in U
\end{aligned}
$$

Where $k$ is an integer number of objective functions, $f_{i}$ is the $i$ th objective function, $\mathbf{u}$ is an input control vector, and $U$ is the feasible set of input control vectors. Although constraints such as vehicle dynamics limit the feasible set of control vectors, multi-objective optimization is challenging and often intractable due to competing objectives and uncertainty in environmental variables such as ocean current conditions and sea-ice cover. For context, consider that the AUG must decide whether or not an additional sensor, such as an imaging sonar, should be turned on. The resource management objective would be minimized if the sensor remains off, but the science objective would be minimized if the sensor is turned on. Not only do the two objectives compete with one another, but the non-commensurate objectives [Schwartz et al., 2002] make it difficult to assess a marginal increase in energy conservation relative to a marginal increase in scientific sampling. Additionally, since environmental states are generally uncertain, it is difficult to provide guaranteed performance for a particular mission plan. Methods designed to account for the relative importance of competing objectives during the mission include the Valuated State Space [Jenkins et al., 2003] method and Interval Programming method [Benjamin et al., 2010].

Rather than considering resource management in the context of global optimization over AUG control variables, resource management is applied to a decoupled version of the problem where a subset of the control variables are provided a-priori by some highlevel mission planner and hotel load optimization policy. The high-level mission planner is responsible for general route parameters such as sequences of waypoints. The hotel load optimization procedure is responsible for duty cycling the AUG hardware components in response to vehicle and environment state. Then, given the high-level mission plan and hotel load, the propulsive system of the AUG can be optimized with respect to energy 
conservation. This low level propulsive optimization is referred to as adaptive velocity control (AVC), which seeks to minimize the transport cost of the AUG. Transport cost is defined in terms of Joules expended per meter traveled, which unlike transport economy [Jenkins et al., 2003], is independent of vehicle weight in order to avoid biases favoring increased vehicle displacements without consideration of supporting infrastructure requirements, as well as associated capital and operating costs.

By decoupling the AUG control problem into hierarchical steps, the propulsive system can be optimized for transport cost independently of other AUG control choices. The control vector $\mathbf{u}$ can then be divided into two sub-components: $\mathbf{u}=\left[\mathbf{u}_{\mathbf{p}}, \mathbf{u}_{\mathbf{h}}\right]^{\top}$, where $\mathbf{u}_{\mathbf{p}}$ refers to control variables of the AUG's propulsive system and $\mathbf{u}_{\mathbf{h}}$ refers to the control variables of the AUG's hotel system. Then, AVC can be expressed as:

$$
\begin{aligned}
& \min _{\mathbf{u}}\left[f_{T C}(\mathbf{u}) \mid \mathbf{u}_{\mathbf{h}}\right] \\
& \text { s.t. } \mathbf{u} \in U
\end{aligned}
$$

Where $f_{T C}$ represents the transport cost of the AUG and is independent of time. For this paper the AUG dynamics are assumed to be well characterized, though it is important to note that several AUG dynamics issues have been observed in the past [Jenkins et al., 2003], [Claus and Bachmayer, 2016]. Additionally, the vertical component of ocean currents is assumed to be negligible compared to the horizontal component.

\subsection{Adaptive Velocity Control}

We define the transport cost objective function $f_{T C}$ to be equal to the total energy expended per distance traveled, $f_{T C}=\frac{\Delta E}{\Delta x}$. Minimizing $f_{T C}$ is the same as maximizing $\frac{1}{f_{T C}}=\frac{\Delta x}{\Delta E}$, which is equivalent to maximizing the total range of the AUG given a finite energy supply. It is important to note that $\Delta E$ refers to the total energy expended by the AUG, not just the energy expended via the AUG propulsive system. This means that the science payload running onboard the AUG affects the optimal travel behavior of the vehicle [Bradley et al., 1992]. 


\subsubsection{Simplified 1D Model}

First, the 1D case is considered where the AUG travels in a straight line and the ocean current is either entirely favorable or entirely adverse, meaning there is no cross-track ocean current. The function to minimize can be written in terms of the instantaneous total power utilized and the over ground velocity $v_{o g}$ of the AUG.

$$
f_{T C}=\frac{P_{t o t a l}}{v_{o g}}
$$

Then, the total power expended by the AUG can be divided into two components: the propulsive load $P_{\text {prop }}$ and the hotel load $P_{\text {hotel }}$. Similarly, the over-ground velocity can be divided into two components: through-water velocity $v_{t w}$, and ocean current velocity $v_{o c}$.

$$
f_{T C}=\frac{P_{\text {prop }}+P_{\text {hotel }}}{v_{t w}+v_{o c}}
$$

Here, the hotel load and ocean currents are assumed to be given. The optimal throughwater velocity of the AUG is given by argument minimum of the transport cost objective function:

$$
v_{t w}^{*}=\underset{v_{t w}}{\arg \min }\left[\frac{P_{\text {prop }}+P_{h o t e l}}{v_{t w}+v_{o c}}\right]
$$

The vehicle velocity given by equation (5.3) is plotted for varying hotel loads and ocean current conditions in Figure 5-1. The figure shows the optimal AUG speed increases with hotel load and decreases with favorable ocean current currents. When there is a strong adverse current, the optimal speed for all hotel load scenarios approaches the AUG's maximum speed of $1.5 \mathrm{~m} \mathrm{~s}^{-1}$, which requires $18.05 \mathrm{~W}$ of power to support.

As mentioned previously, minimizing $f_{T C}$ is the same as maximizing $\frac{1}{f_{T C}}=\frac{\Delta x}{\Delta E}$, which is equivalent to maximizing the total range of the AUG given a finite energy supply. To explore this visually, two plots are shown: Figure 5-2 shows the maximum AUG range as a function of hotel load while neglecting the affect of ocean currents, and Figure 5-3 shows the maximum AUG range as a function of ocean current conditions for both the transit and survey hotel load scenarios.

Figure 5-2 shows that the AUG can travel in excess of $7500 \mathrm{~km}$ if operating in transit mode for the entire mission, and that the AUG can travel in excess of $2500 \mathrm{~km}$ if operating 
A)

Energy Optimal Glider Speed

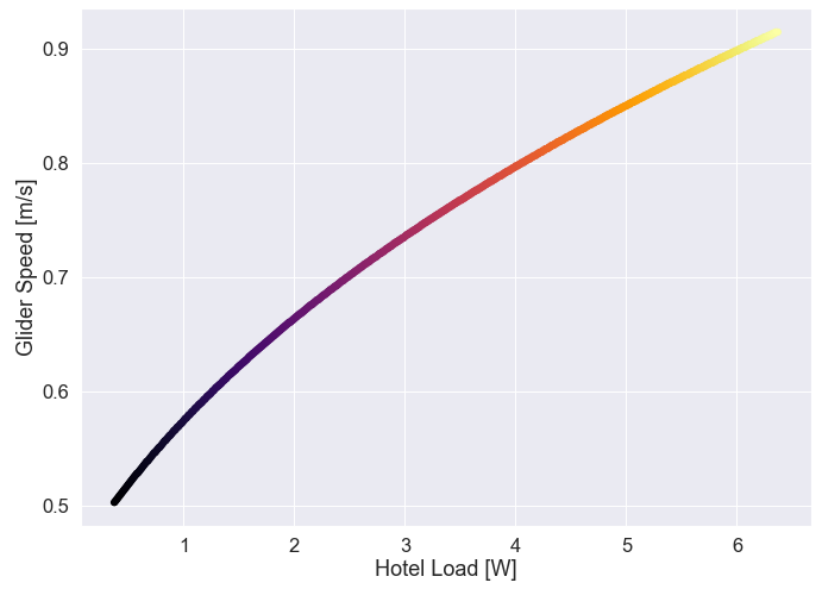

B)

Energy Optimal Glider Speed

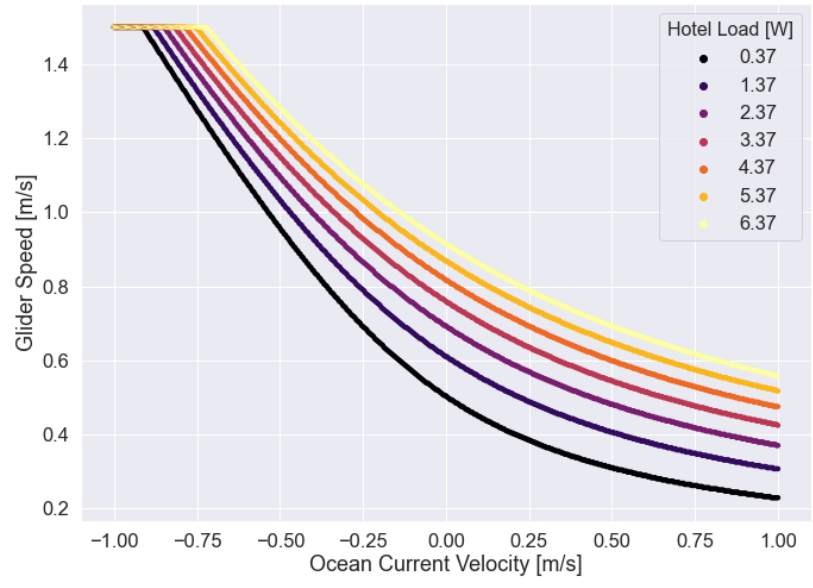

Figure 5-1: Energy optimal propulsive speed as a function of hotel load and ocean current conditions for the $1 \mathrm{D}$ case. Color in both subplots indicates hotel load, where hotel load values vary between transit mode $(0.37 \mathrm{~W})$ and survey mode $(6.37 \mathrm{~W})$.

in survey mode for the entire duration. However, it is important to approach these range estimates with caution because they neglect the effect of ocean currents, where adverse or cross-track ocean currents can severely hinder the range performance of the AUG.

Figure 5-3 shows that the AUG range changes drastically dependent on the ocean current conditions. For example, for the transit hotel load case, the approximate AUG range for $1 \mathrm{~m} \mathrm{~s}^{-1}$ favorable current, $0 \mathrm{~m} \mathrm{~s}^{-1}$ current, and $1 \mathrm{~m} \mathrm{~s}^{-1}$ adverse current are $27000 \mathrm{~km}$, $7500 \mathrm{~km}$, and $1000 \mathrm{~km}$, respectively. Although it is unrealistic for the AUG to experience entirely favorable or entirely adverse ocean currents of $1 \mathrm{~m} \mathrm{~s}^{-1}$ magnitude for the entire mis- 


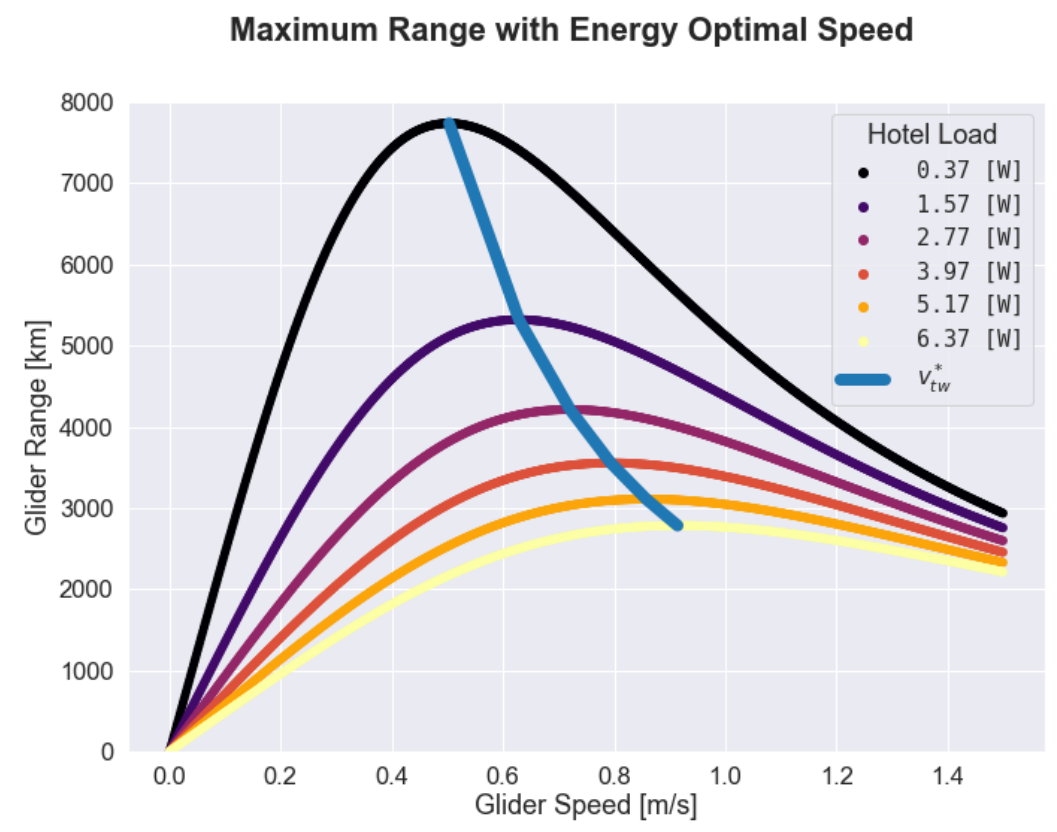

Figure 5-2: AUG range as a function of vehicle through-water speed and hotel load. This plot assumes an energy supply of $10 \mathrm{~kW}$ h and ignores the effect of ocean currents. The blue curve shows how the range-maximizing through-water speed of the AUG increases with increasing hotel load, echoing the result of equation (5.3).
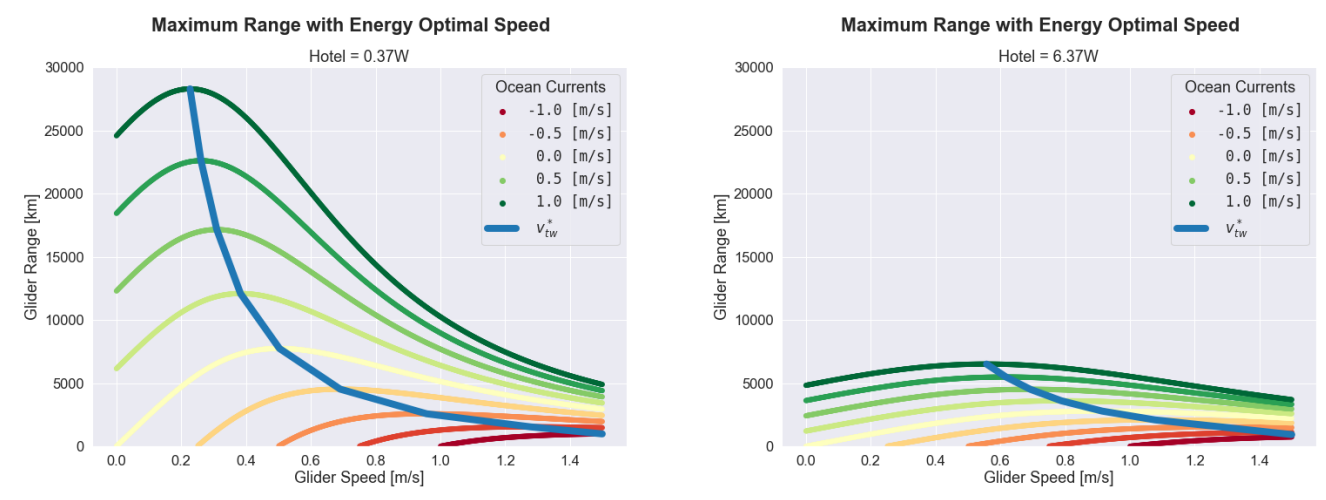

Figure 5-3: AUG range as a function of vehicle through-water speed and ocean current conditions, shown for the transit hotel load in the left subplot and the survey hotel load in the right subplot. This plot assumes an energy supply of $10 \mathrm{~kW} \mathrm{~h}$. The blue curve shows how the range-maximizing through-water speed of the AUG increases with decreasing favorable ocean currents, echoing the result of equation (5.3).

sion duration, this analysis provides an upper and lower bound of AUG range performance. 


\subsubsection{Generalized 3D Model}

For the general 3D case, the impact of operating the buoyancy engine is accounted for and a $3 \mathrm{D}$ representation of the ocean currents is utilized. The usage of the buoyancy engine impacts the AUG through-water speed and the AUG energy budget. The through-water speed can now be treated as the sum of components from the thruster $v_{\text {prop }}$ and the buoyancy engine $v_{\text {buoy }}$, where the buoyancy engine component is dependent on the AUG pitch angle $\phi$ and is well approximated with the following second order polynomial:

$$
v_{\text {buoy }}=(1.13 \mathrm{e}-1)+(1.55 \mathrm{e}-2) \phi-(2.17 \mathrm{e}-4) \phi^{2}
$$

Then, the through-water speed is given by the summation of components from the thruster and the buoyancy engine. Note that the resulting through-water speed acts in the AUG heading direction $\boldsymbol{\psi}$, but has a declination of $\xi$ with respect to the horizontal plane, where $\xi$ is the AUG glide angle given by the sum of pitch and angle of attack: $\xi=\phi+\alpha$. The through-water speed can be expressed as:

$$
v_{t w}=v_{\text {prop }}+v_{\text {buoy }}
$$

Velocity vectors are denoted with boldface text $\mathbf{v}$, while speeds are denoted with regular text $v$, where speed is given by the L2 norm of the velocity vector $v=\|\mathbf{v}\|$. The vertical component of through-water speed $v_{t w} \sin \xi$ can now be used in conjunction with the vertical component of the ocean current vector to express the average power draw of the buoyancy engine, and the horizontal component of through-water speed $v_{t w} \cos \xi$ can be used in conjunction with the horizontal component of the ocean current vector to express the over-ground speed.

The average power draw of the buoyancy engine is dependent on the following parameters: dive-to depth $z_{\text {dive }}$, climb-to depth $z_{\text {climb }}$, AUG pitch $\phi$, AUG glide angle $\xi$, the vertical component of the ocean current vector $w_{o c}$, the energy cost of operating the ballast pump during bottom inflections $E_{\text {pump }}$, and the percent of ballast volume pumped by buoyancy engine $\gamma$. Note that $E_{\text {pump }}$ is generally a function of dive-to depth $z_{\text {dive }}$. Here, $z_{\text {dive }}$ and $z_{\text {climb }}$ define the depth band of AUG, which are now assumed to bed given. In Section 5.4, a depth band optimization policy is discussed which takes into account the shear structure within the water column. The selection is $\gamma$ is discussed further in Section 5.5. Let $\bar{w}_{o c}$ 
represent the average vertical component of the ocean current velocity over the depth band. Now, the average power consumption of the buoyancy engine can be written as a change in energy over a change in time:

$$
P_{\text {buoy }}=\frac{\Delta E}{\Delta t}=\frac{\gamma E_{\text {pump }}}{\left[\frac{2\left(z_{\text {dive }}-z_{\text {climb }}\right)}{v_{t w} \sin \xi+\bar{w}_{o c}}\right]}
$$

Figure 5-4 explores equation (5.6) visually, where the average power draw of the buoyancy engine is shown as a function of depth band size and AUG pitch angle, where the other variables in equation (5.6) are assumed given. The figure shows that $P_{b u o y}$ grows exponentially for small depth bands and large pitch angles. This power draw equation is utilized more formally in Section 5.4 when AUG depth band is optimized with respect to transport cost. For the remainder of this section, it is assumed that the $P_{\text {buoy }}=1.5 \mathrm{~W}$, the average buoyancy engine power for a $500 \mathrm{~m}$ depth band at $12^{\circ}$ pitch.

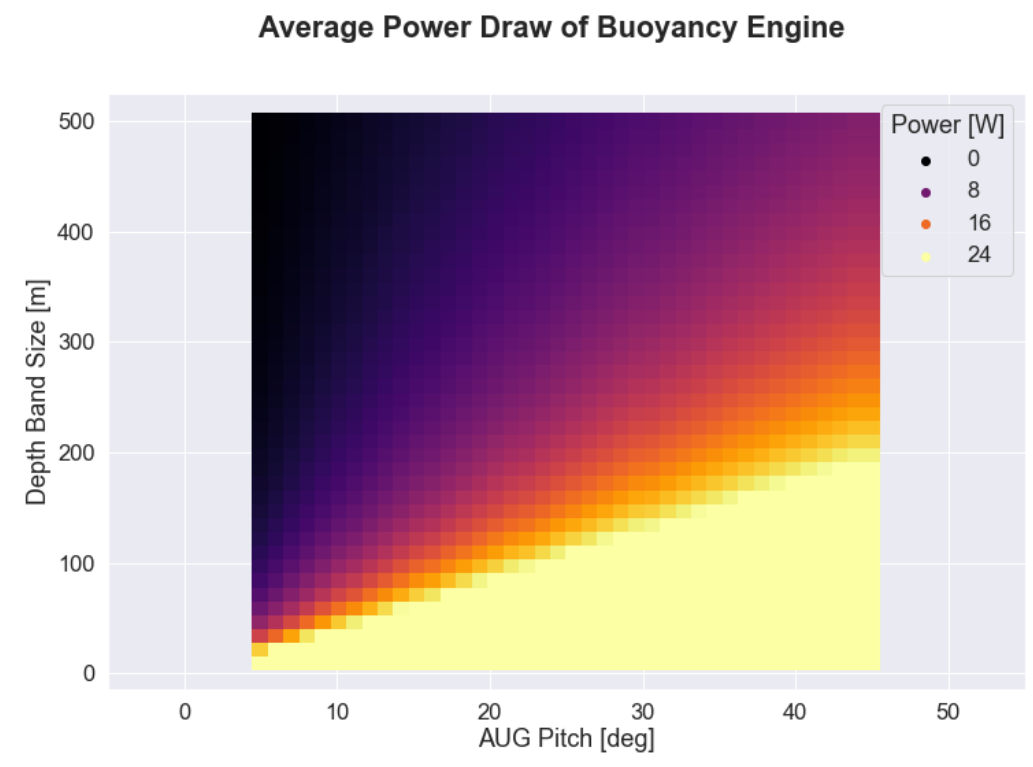

Figure 5-4: Average power draw of the buoyancy engine as a function of depth band size $z_{\text {dive }}-z_{\text {climb }}$ and AUG pitch angle $\phi$ assuming zero angle of attack and negligible vertical component of ocean currents. Since the average power grows exponentially for small depth bands and large pitch angles, exceeding $1000 \mathrm{~W}$ in some cases, the plot is capped at $24 \mathrm{~W}$ for easier interpretation.

Now that the buoyancy engine has been accounted for in terms of its contribution to AUG through-water velocity and AUG energy consumption, over-ground velocity can be written in terms of arbitrary 3D ocean currents so that AVC can be performed. Ocean 
currents are no longer restricted to being co-linear with the vehicle path as was true in the $1 \mathrm{D}$ case. Let $\boldsymbol{\psi}$ be the AUG heading, $\eta$ be the ocean current heading, $\delta=\eta-\boldsymbol{\psi}$ be the relative alignment of the ocean currents with respect to the AUG heading. If $\delta=0^{\circ}$, ocean currents are entirely favorable and if $\delta= \pm 180^{\circ}$ ocean currents are entirely adverse. For angle magnitudes between $0^{\circ}$ and $180^{\circ}$, the AUG must overcome a cross-track current $v_{o c} \sin \delta$ to maintain the desired heading. After accounting for the cross-track current, the co-linear component of the ocean current $v_{o c} \cos \delta$ can be considered, yielding the following equation for optimal AUG speed over-ground:

$$
v_{o g}=\left(\left(v_{t w} \cos \xi\right)^{2}-\left(v_{o c} \sin \delta\right)^{2}\right)^{\frac{1}{2}}+v_{o c} \cos \delta
$$

Note that for general 3D ocean current vectors, the vertical component of ocean current velocity does not affect speed over-ground because it is perpendicular to the horizontal ground plane. However, the vertical component of ocean current affects the power consumption of the buoyancy engine, as shown in equation (5.6).

Leveraging equation (5.7), the optimal through-water speed can be written, which serves as the AVC control policy in the general 3D case:

$$
v_{t w}^{*}=\underset{v_{t w}}{\arg \min }\left[\frac{P_{\text {prop }}+P_{b u o y}+P_{\text {hotel }}}{v_{o g}}\right]
$$

Where $P_{\text {prop }}$ and $P_{\text {buoy }}$ are dependent on $v_{t w}$, and $P_{h o t e l}$ is independent of $v_{t w}$. Equation (5.8) serves as the general AVC policy that minimizes AUG transport cost in response to ocean current conditions and hotel load.

Since the optimal through-water velocity is designed to oppose cross-track currents while maintaining forward progress, AVC inherently includes active steering to minimize crosstrack and long-track drift caused by ocean currents. Thus, the active steering aspect of AVC leads to a further reduction in transport cost compared to standard control methods that would otherwise require additional time and energy to correct for navigation error caused by ocean current drift. Although this approach is not a path planning method since it does not modify the horizontal path plan, it is used as a control policy to minimize transport cost along a pre-specified trajectory. In the event that strong adverse or cross-track currents are encountered, the AUG may not be capable of pursuing the intended path, and contingency planning may be initiated by the higher-level mission planning system. The results of AVC 
are shown in Figure 5-5.
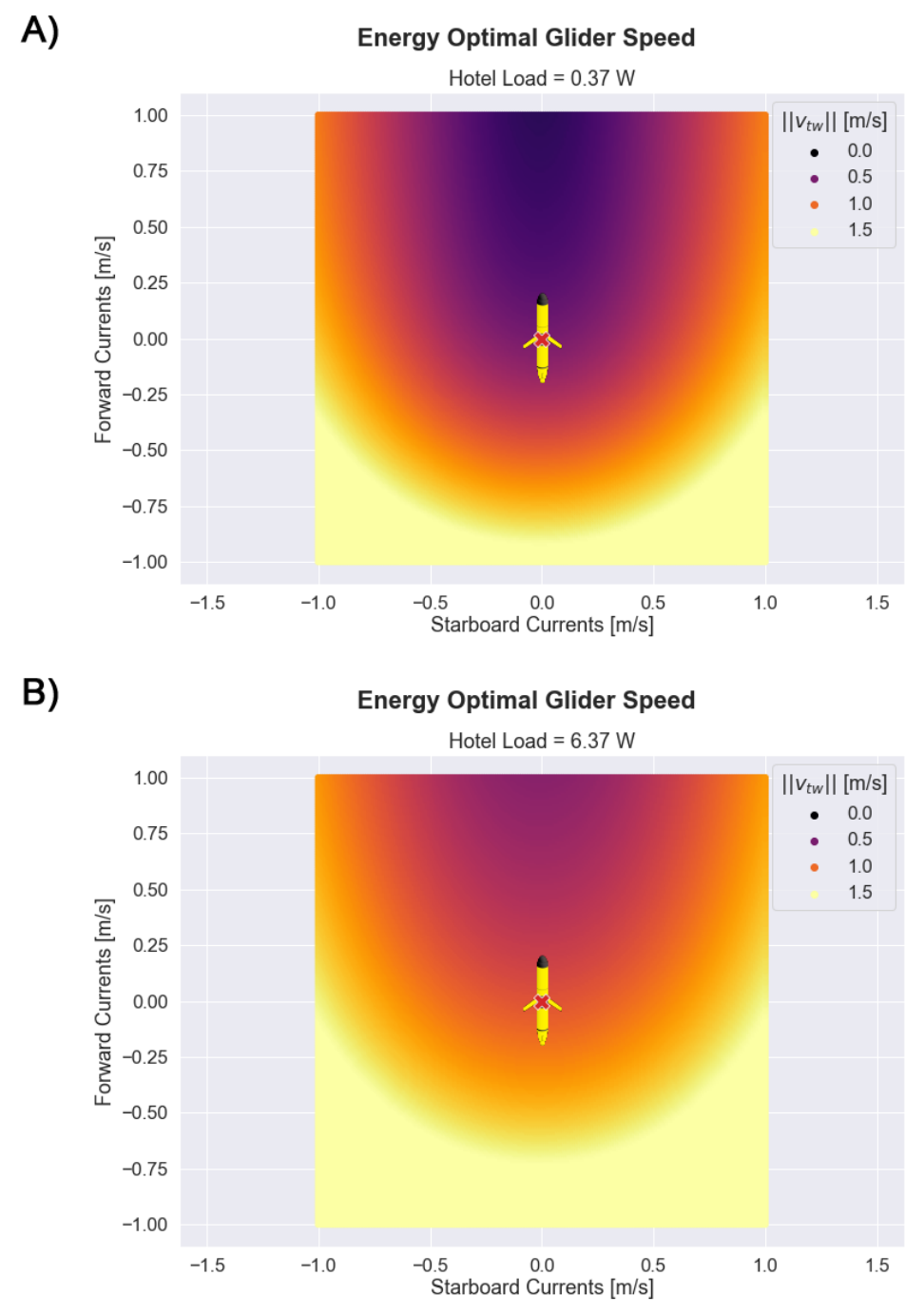

Figure 5-5: AVC given horizontal ocean current speeds $\leq 1 \mathrm{~m} \mathrm{~s}^{-1}$, where the vertical component is assumed to be negligible. In each subplot, the $\mathrm{x}$-coordinate represents the cross-track component of the ocean current and the y-coordinate represents the co-linear component of the ocean current. The color shown in both subplots represents the optimal AUG throughwater speed for transit and survey hotel loads given by the AVC control policy in equation (5.8).

Subplots A and B of Figure 5-5 echo the results obtained in the 1D case, namely that it is energetically beneficial for the AUG to speed up in the presence of adverse currents or increased hotel loads and to slow down in the presence of favorable currents or decreased hotel loads. Additionally, the figure conveys the impact of cross-track ocean currents: the AUG must increase its velocity significantly to counteract cross-track currents while still 
maintaining forward progress.

To perform AVC, ocean current velocities must be known, and as a result, the DVL and science computer must be used to actively estimate these ocean currents. Thus, the AVC control policy is not serviceable for conventional transit mode operation because conventional transit hotel load does not include DVL and science computer operation. This presents a trade-off that must be considered while transiting: utilize the conventional method of minimizing transit hotel load and forfeit the ability to perform AVC, or increase the hotel load during transit by operating the DVL and science computer to enable AVC. The value of this trade-off is dependent on environmental state. For example, if ocean current velocities have magnitude zero, it is unnecessary to operate the DVL and science computer because AUG propulsion cannot be adjusted in response to ocean currents.

Decrease in Transport Cost if DVL used while Transiting

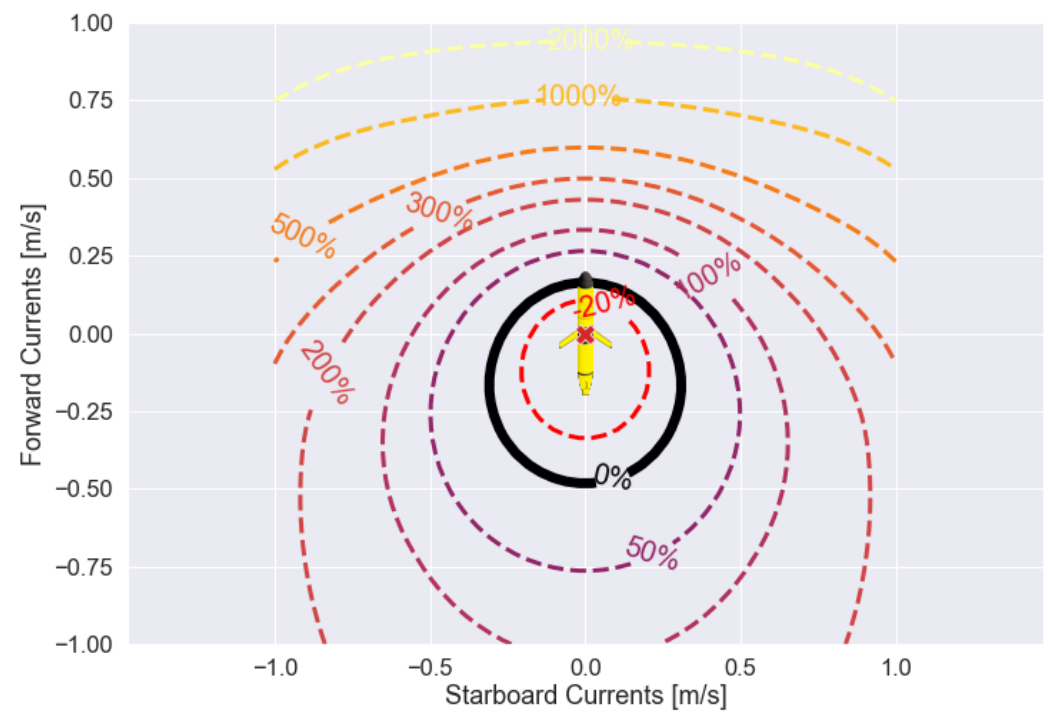

Figure 5-6: Transport cost improvement obtained by operating the DVL while transiting. By operating the DVL during transit, the AUG can perform AVC, which includes active steering to avoid cross-track and long-track drift caused by ocean currents. For ocean currents of small magnitudes, transport cost increases when the DVL is operated during transit.

Figure 5-6 explores this trade-off by showing the percent decrease in transport cost when using the DVL and science computer while transiting, when compared to the default transit hotel configuration. The black solid line indicates when the AUG transport cost is energetically equivalent between transiting with or without the DVL and science computer 
being operated. Inside the black solid line the transport cost associated with use of the DVL is increased and outside the black solid line the transport cost is decreased. Interestingly, the environmental state that leads to the greatest decrease in transport cost when using the DVL involves favorable ocean current with no cross-track component. This is largely due to the active steering component of AVC: the default transit mode overshoots the intended waypoint due to long-track drift caused by favorable ocean currents, requiring a navigation correction that must then backtrack while fighting against ocean currents that are now entirely adverse to reach the target waypoint.

\subsection{Exploitative Depth Band Selection}

With sufficient available water depth, the AUG can perform adaptive depth band selection to exploit velocity shear structure in the water column. This process is referred to as exploitative depth band selection (EDBS). As an example, if the water column contains two water mass layers such that one layer has a favorable current and the other layer has an adverse current, it may be energetically optimal for the AUG to confine itself to the favorable layer instead of travel throughout the full available water column. The downside of this narrowing of depth band is an increased frequency of inflections, which corresponds with an increased usage of the energetically expensive buoyancy pump. This trade-off presents an optimization that can be exploited to further improve the transport economy of the AUG if water column estimates are available.

Let $v_{o c, z}$ and $\eta_{z}$ represent the ocean current magnitude and direction at depth $z$, which are assumed to have been estimated via the VSP method discussed in Section 4.2.2. Then, given the heading of the AUG $\psi$ and the hotel load $P_{\text {hotel }}$, the ocean current heading offset is given by $\delta_{z}=\eta_{z}-\psi$ and the optimal through-water speed $v_{t w, z}^{*}$ at depth $z$ is given by equation (5.8). The over-ground speed $v_{o g, z}$ at depth $z$ is given by equation (5.7), where $v_{o g, z}$ encodes the relative alignment of the ocean currents with respect to AUG heading.

Let $\phi$ be the pitch angle of the AUG and $P_{\text {prop }, z}$ be the instantaneous propulsive power

of the hybrid thruster which is a function of the optimal through-water speed $v_{t w, z}^{*}$ via equation (2.1). Then, let $z_{d}$ and $z_{c}$ be the dive-to and climb-to depths of the AUG, which are the parameters that define the depth band of the AUG. The optimal depth band can then be selected by taking the argument minimum of the transport cost function, where the 
transport cost is now a function of depth band. Here, three sources of energy expenditure are considered: the buoyancy engine ballast pump, the hybrid thruster, and the hotel load. The buoyancy pump is modeled by a fixed energy cost $E_{\text {pump }}$ in units of J, which in general can be modeled as a function of dive-to depth $z_{d}$. The propulsive unit and hotel load are modeled in units of $\mathrm{W}$, so they must be integrated over time to yield a quantity of energy. To perform this integration, integration is performed over the depth band defined by $z_{d}$ and $z_{c}$, and the variable of integration $d t$ is replaced using through-water vertical speed and the vertical component of the ocean current, both at depth $z: v_{t w, z} \sin \xi+w_{o c, z}=\frac{d z}{d t}$. Similarly, the over-ground speed $v_{o g, z}$ must be integrated over the depth band to give the horizontal distance traveled while moving through the depth band. Again, $\gamma$ is the percent of ballast volume pumped by buoyancy engine. The optimal depth band can then be written as the argument minimum of the transport cost function:

$$
\operatorname{DepthBand}^{*}=\underset{z_{d}, z_{c}}{\arg \min }\left[\frac{\frac{\gamma E_{\text {pump }}}{2}+\int_{z_{d}}^{z_{c}} \frac{P_{\text {prop }, z}+P_{\text {hotel }}}{w_{t w, z}+w_{o c, z}} d z}{\int_{z_{d}}^{z_{c}} \frac{v_{o g, z}}{w_{t w, z}+w_{o c, z}} d z}\right]
$$

The expression in equation (5.9) concisely captures an intriguing trade-off between the energy cost of using the buoyancy pump during bottom inflections and the two watercolumn-integrated variables: power and over-ground speed. Note that the energy cost of the buoyancy pump is divided by two because the pump is only utilized during bottom inflections. To showcase the expressivity of this result, exemplar scenarios are presented. If a vehicle has negligible buoyancy pump cost, $E_{\text {pump }} \approx 0$, then the EDBS will simply select a depth band of infinitesimally minimal height that is centered about the most favorable current in the water column. A similar conclusion can be made for vehicles with exorbitantly high hotel loads: the water-column-integrated hotel load dominates the buoyancy pump cost regardless of the size of the depth band, so the optimal depth band centers on the most favorable current in the water column. On the other hand, if a vehicle has excessively high buoyancy pump energy cost, EDBS will maximize the depth band height to minimize the frequency of using the energetically expensive buoyancy pump. For the case of uniform water column currents in depth, EDBS again maximizes the size of the depth band because there is no shear layer to exploit. Finally, when there is high contrast between velocity shear layers in the water column, it may be optimal for the vehicle to restrict itself to a 
narrowed depth band within the most favorable shear layer.

Since EDBS considers all valid combinations of dive-to and climb-to depths, the policy will always perform at least as well as the default behavior of utilizing the maximum available water column. To improve the computational speed of EDBS, additional constraints can be used to bound the set of valid dive-to and climb-to combinations.

\subsection{Adaptive Duty Cycling}

Although hotel load is often considered as a static differential between transit mode and survey mode, individual hardware components can be optimized with respect to vehicle and environment state. In this section, the duty cycle reduction of three of the most powerintensive hotel components is considered: the Doppler Velocity Log (DVL), the Mechanical Scanning Imaging Sonar (MSIS), and the Back-seat Driver computer (BSD). Additionally, buoyancy engine power can be lowered by reducing pump volume of the buoyancy engine and by pursuing a shallower pitch angle of $5^{\circ}$ to limit frequency of inflections. This reduction of hotel components in response to science and navigation requirements is referred to as adaptive duty cycling (ADC).

The constraint that limits the minimum DVL duty cycle pertains to AUG localization and ocean current velocity estimation. For both of these functions to be performed with reasonable accuracy, it is necessary for the DVL to make at least 10 water-track observations for each vertical meter traveled by the AUG. At a nominal speed of $0.75 \mathrm{~m} \mathrm{~s}^{-1}$ at $5^{\circ}$ pitch, the DVL must take samples at a frequency of $0.65 \mathrm{~Hz}$. The nominal sampling frequency for the DVL at $100 \%$ duty cycle is approximately $2 \mathrm{~Hz}$. Therefore, to minimize DVL usage while meeting localization and state estimation requirements, the DVL can be operated at a duty cycle of $32 \%$.

For duty cycle reduction of the MSIS, the science constraint enforces that sea-ice classifications are made at a spatial resolution of $50 \mathrm{~m}$. From Section 3.7.2, each sea-ice sonar scan takes $15 \mathrm{~s}$ to record. At a nominal speed of $0.75 \mathrm{~m} \mathrm{~s}^{-1}$ at $5^{\circ}$ pitch, it takes the AUG approximately $67 \mathrm{~s}$ to travel $50 \mathrm{~m}$. Thus, to minimize the usage of the MSIS while adhering to the sea-ice sampling constraint, duty cycle of the MSIS can be set to $22 \%$.

Finally, the BSD computer must be in operation to command the hotel load sensors such as the DVL and the MSIS, as well as process sensor data from these instruments in 
real-time and send AUG control commands as necessary. The BSD computer is estimated to operate at $50 \%$ duty cycle while maintaining proper coordination of AUG sensors, data processing, and AUG control.

The results of duty cycle reduction is summarized in Figure 5.1. The cumulative power draw for the hotel load has been reduced from $5.4 \mathrm{~W}$ to $1.5 \mathrm{~W}$, reducing the total AUG power draw for survey operations from $6.37 \mathrm{~W}$ to $2.47 \mathrm{~W}$. Further reduction in energy consumption can be realized when using the hybrid thruster by decreasing the pumped displacement volume of the buoyancy engine. For example if the buoyancy engine, which requires approximately $2.95 \mathrm{~W}$ h per bottom inflection for full volume displacement, instead pumps just $20 \%$ of its available volume, the duty cycle would decrease by $80 \%$ resulting in $0.59 \mathrm{Wh}$ per bottom inflection. Despite glide efficiency decreasing with decreased pitch angle, additional duty cycle minimization may be obtained by decreasing the vehicle pitch angle to shallower angles around $5^{\circ}$ that remain above the critical stall angle. While the exact percentages of duty cycle reduction are dependent on specific operating constraints of hardware components, significant energy reduction can be achieved through adaptive minimization of buoyancy engine and hotel system power draw without sacrificing science or mission requirements. As illustrated in the discussion section, a duty cycle reduction of this magnitude can lead to substantial improvements to AUG range and mission duration.

\begin{tabular}{|c|c|c|c|}
\hline $\begin{array}{c}\text { Hardware } \\
\text { Component }\end{array}$ & $\begin{array}{c}\text { Default } \\
\text { Power Draw } \\
{[\mathrm{W}]}\end{array}$ & $\begin{array}{c}\text { Reduced } \\
\text { Duty Cycle } \\
{[\%]}\end{array}$ & $\begin{array}{c}\text { Reduced } \\
\text { Power Draw } \\
{[\mathrm{W}]}\end{array}$ \\
\hline DVL & 2.00 & 32.0 & 0.64 \\
\hline MSIS & 3.00 & 22.0 & 0.66 \\
\hline BSD & 0.40 & 50.0 & 0.20 \\
\hline
\end{tabular}

Table 5.1: Review of hardware components that can be adaptively duty cycled during mission to reduce power expenditure. For resource-constrained AUG missions in the Arctic, operation of these instruments can be reduced to save energy while still meeting mission safety and science data collection requirements.

\subsubsection{Satellite Data Transfer}

In this AUG architecture an MSIS collects data at a rate of approximately 8 kilobytes per second and requires 15 seconds to complete a scan across the most informative $\pm 60^{\circ}$ sector for sea-ice classification. This means that each sea-ice scan completed by the MSIS is ap- 
proximately 125 kilobytes in size. In order to accommodate the decision input requirements of the AUG's embedded contingency planner and the bandwidth limitations of the satellite communications, an automated sea-ice characterization process can perform a hierarchical analysis of sea-ice features, including: presence or absence of sea-ice, sea-ice thickness, and sea-ice roughness. From these three sea-ice features, sea-ice scan measurements can be categorized as a discrete set of sea-ice types. More details on this acoustics-based sea-ice characterization scheme are included in Chapter 3.

The satellite communication system requires $6.5 \mathrm{~W}$ of power when active. At the signalto-noise levels typically available in the Arctic, the satellite communication system would be expected to transmit no more than 240 bits per second reliably. Raw acoustic scan data for one sea-ice observation is 125 kilobytes whereas the compressed sea-ice characterization information can be encoded in 8 bytes, which constitutes a four orders of magnitude size reduction. This results in an equivalent decrease in power required to transmit sea-ice information via satellite communication. It is noteworthy that even if the AUG had access to unlimited power, the data transfer rate for transmitting raw data would eclipse the collection rate by over 1.5 orders of magnitude, meaning that each minute of data collected would require over an hour of satellite-base transmittal. Thus, based on energy budget alone, automated sea-ice interpretation and information compression is a necessity for sonar-based AUG survey of Arctic sea-ice.

\subsection{Results}

Our methods for propulsive control with AVC and ocean current estimation with VSP are evaluated using AUG sea-trial data from a November 2019 deployment within an active submarine volcano in the Southern Aegean Sea. For this set of experiments, a Slocum AUG was used as a reconnaissance platform to provide initial surveys of the Kolumbo caldera crater to inform subsequent ROV mission planning. During these missions the AUG relied on its default DR process, which included a static current correction that calculated a temporal and depth-averaged water column current based on discrepancy between the GPS and DR localization estimates during its prior dive. This DR with depth-averaged current correction (DR-DACC) does not directly observe ocean current velocities and is unable to account for temporally dynamic currents or biasing caused by variability in the AUG's 
depth band. Six AUG missions are selected to evaluate the AVC method and a subset of these missions are used to illustrate the performance of the VSP ocean current estimation method.

\subsubsection{Adaptive Velocity Control}

Based on the demonstrated validity of the ocean current estimation process, the potential utility of AVC can now be explored. Although the AVC method was not running onboard the AUG, potential performance benefits of using this control method can be evaluated through offline analysis of three AUG control policies: constant velocity buoyancy drive, constant velocity hybrid drive, and adaptive velocity hybrid drive.

Figure 5-7 shows the behavior of the AVC method when applied to the Dive A series previously described in Figure 4-4. As shown in Figure 5-7, the AUG increases its propulsive speed in times of adverse or cross-track currents, whereas the AUG decreases propulsive speed in times of favorable currents. Since the majority of Dive A consisted of adverse ocean currents, AVC characteristically commands the vehicle to a faster through-water speed than the constant velocity controller. While traveling at higher speeds increases the average power consumption of the AUG, the mission duration is decreased such that the total power consumption is reduced. Thus AVC is able to adaptively respond to environmental state.

Table 5.2 shows the results of the three velocity controllers when applied to six of the AUG missions during a series of dives in November 2019 in the Southern Aegean Sea. The velocity controllers are evaluated with respect to three metrics: mission duration, average power draw, and transport economy. Since the transport economy metric normalizes for the distance traveled during the mission, it is the most informative metric when comparing across the different missions.

As seen in the table, the hybrid AUG achieves a transport cost that is approximately twice as efficient as conventional buoyancy drive. Although the buoyancy engine alone yields the lowest average power draw, the slower speeds of the buoyancy engine travel lead to significantly longer mission times, which leads to less overall energy efficiency.

Additionally, because the measured water column currents were consistently non-zero, the adaptive hybrid controller reliably outperforms the constant hybrid controller, regardless of the magnitude and direction of water column currents. This result confirms that transport 

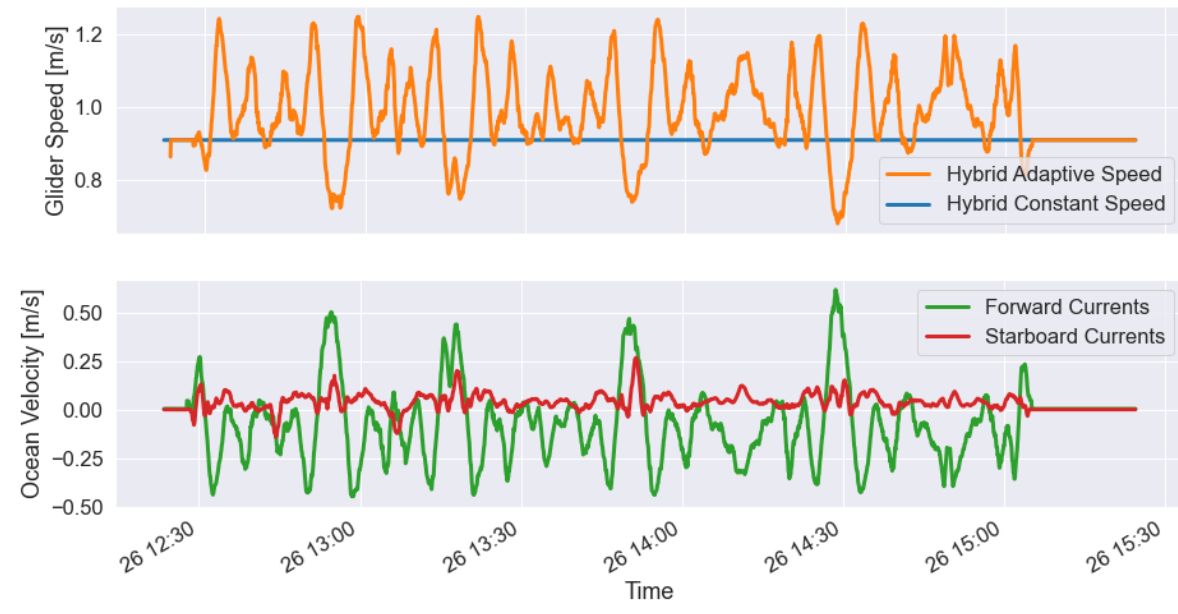

Figure 5-7: AVC when applied to Dive A of the November 2019 deployment in the Southern Aegean Sea. The Hybrid Adaptive Speed curve shows the optimal AUG speed over time, which responds to varying ocean current conditions. The Hybrid Constant Speed is only optimized with respect to hotel load, so it does not change during the mission. The Forward Currents and Starboard Currents during mission are estimated via the VSP method.

cost can be independently optimized as a decoupled component of the AUG mission planning problem. The adaptive hybrid controller performs $4.6 \%$ more efficiently than the constant hybrid controller, and in instances of strong adverse ocean currents for large portions of the mission, such as in Dive A, the adaptive speed controller performs as much as $8.2 \%$ better.

Therefore, by using acoustic sensing to estimate ocean currents in real-time onboard the AUG, the vehicle can perform AVC to reap significant improvements in energy efficiency. In addition to AVC, further energy management improvements can be realized by modulating total displaced volume pumped by the buoyancy engine in relation to thruster power, and depth band can be continuously adjusted to maximally exploit favorable shear layers in the water column.

\subsubsection{Exploitative Depth Band Selection}

In this section, results of applying the EDBS control policy are shown for AUG dive data obtained during the November 2019 research deployment in the Southern Aegean Sea. The plots shown in Figure 5-8 and Figure 5-9, along with the data shown in Table 5.3, make the following AUG operation assumptions: the AUG is operating in reduced hotel survey mode 


\begin{tabular}{|c|c||c|c|c|}
\hline \multirow{2}{*}{$\begin{array}{c}\text { Mission } \\
\text { Name }\end{array}$} & \multirow{2}{*}{$\begin{array}{c}\text { Distance } \\
\text { Traveled }[\mathrm{m}]\end{array}$} & \multicolumn{3}{|c|}{ Mission Time [min] } \\
\cline { 3 - 5 } & $\begin{array}{c}\text { Buoyancy } \\
\text { Constant } \\
\text { Speed }\end{array}$ & $\begin{array}{c}\text { Hybrid } \\
\text { Constant } \\
\text { Speed }\end{array}$ & $\begin{array}{c}\text { Hybrid } \\
\text { AVC } \\
\text { Speed }\end{array}$ \\
\hline Dive A & 6063 & 328 & 143 & $\mathbf{1 1 5}$ \\
\hline Dive B & 2520 & 114 & 47.8 & $\mathbf{4 5 . 7}$ \\
\hline Dive C & 4910 & 243 & 92.2 & $\mathbf{8 9 . 4}$ \\
\hline Dive D & 2050 & 97.7 & 40.9 & $\mathbf{3 8 . 0}$ \\
\hline Dive E & 905 & 64.3 & 19.2 & $\mathbf{1 7 . 3}$ \\
\hline Dive F & 985 & 74.8 & 21.6 & $\mathbf{1 8 . 9}$ \\
\hline
\end{tabular}

\begin{tabular}{|c|c||c|c|c|}
\hline \multirow{2}{*}{$\begin{array}{c}\text { Mission } \\
\text { Name }\end{array}$} & \multirow{2}{*}{$\begin{array}{c}\text { Distance } \\
\text { Traveled [m] }\end{array}$} & \multicolumn{3}{|c|}{ Average Power Draw [W] } \\
\cline { 3 - 5 } & $\begin{array}{c}\text { Buoyancy } \\
\text { Constant } \\
\text { Speed }\end{array}$ & $\begin{array}{c}\text { Hybrid } \\
\text { Constant } \\
\text { Speed }\end{array}$ & $\begin{array}{c}\text { Hybrid } \\
\text { AVC } \\
\text { Speed }\end{array}$ \\
\hline Dive A & 6063 & $\mathbf{7 . 9}$ & 13.2 & 15.1 \\
\hline Dive B & 2520 & $\mathbf{1 6 . 2}$ & 21.6 & 22.0 \\
\hline Dive C & 4910 & $\mathbf{1 1 . 1}$ & 16.5 & 16.8 \\
\hline Dive D & 2050 & $\mathbf{1 1 . 1}$ & 16.5 & 17.2 \\
\hline Dive E & 905 & $\mathbf{1 6 . 2}$ & 21.6 & 22.8 \\
\hline Dive F & 985 & $\mathbf{1 6 . 2}$ & 21.6 & 23.0 \\
\hline
\end{tabular}

\begin{tabular}{|c|c|c|c|c|}
\hline \multirow{2}{*}{$\begin{array}{l}\text { Mission } \\
\text { Name }\end{array}$} & \multirow{2}{*}{$\begin{array}{c}\text { Distance } \\
\text { Traveled }[\mathrm{m}]\end{array}$} & \multicolumn{3}{|c|}{ Transport Cost $\left[\mathrm{J} \mathrm{m}^{-1}\right]$} \\
\hline & & $\begin{array}{c}\text { Buoyancy } \\
\text { Constant } \\
\text { Speed }\end{array}$ & $\begin{array}{c}\text { Hybrid } \\
\text { Constant } \\
\text { Speed }\end{array}$ & $\begin{array}{l}\text { Hybrid } \\
\text { AVC } \\
\text { Speed }\end{array}$ \\
\hline Dive $\mathrm{A}$ & 6063 & 25.5 & 18.5 & 17.1 \\
\hline Dive B & 2520 & 43.9 & 24.5 & 23.9 \\
\hline Dive $\mathrm{C}$ & 4910 & 32.9 & 18.6 & 18.3 \\
\hline Dive D & 2050 & 31.7 & 19.8 & 19.1 \\
\hline Dive $\mathrm{E}$ & 905 & 69.1 & 27.5 & 26.2 \\
\hline Dive F & 985 & 73.8 & 28.4 & 26.5 \\
\hline \multicolumn{2}{|c|}{ Mean Transport Cost } & $46.1 \pm 18.7$ & $22.9 \pm 4.1$ & $21.9 \pm 3.8$ \\
\hline
\end{tabular}

Table 5.2: Offline performance analysis between three AUG velocity control policies applied to November 2019 Southern Aegean Sea missions previously mentioned: constant velocity with the buoyancy engine alone, constant velocity with the hybrid buoyancy and thruster system, and AVC with the hybrid buoyancy and thruster system. The control methods are evaluated with respect to three metrics: mission time, average power draw, and transport cost. The most important metric for AUG energy efficiency, transport cost, shows that in this mission context the hybrid AUG performs approximately twice as well compared to the buoyancy engine alone, and the AVC method reliably outperforms the constant velocity controller.

$(2.12 \mathrm{~W})$, the AUG is operating at $5^{\circ}$ pitch angle, and the ballast pump volume is set to $20 \%$ of maximum ballast volume. The derivation of these operating conditions is discussed 
in Section 5.5. The Figures display the results of the EDBS policy as applied to two separate dives during the research deployment: Dive B with strong velocity shear structure in the water column, and Dive F with more uniform structure in the water column.


Figure 5-8: EDBS as applied to Dive B of the November 2019 research deployment in the Southern Aegean Sea. The EDBS method is applied at heading intervals of $45^{\circ}$. For each AUG heading considered, the left subplot shows the transport cost as a function of depth band, where the yellow color indicates low transport cost and the blue color indicates high transport cost. Similarly, each right subplot shows the water column velocities at $1 \mathrm{~m}$ depth intervals with the resulting optimal depth band highlighted in orange.

The results displayed in Figure 5-8 and Figure 5-9 are displayed numerically in Table 5.3. In the table, the EDBS method is compared to the default behavior of utilizing the full 

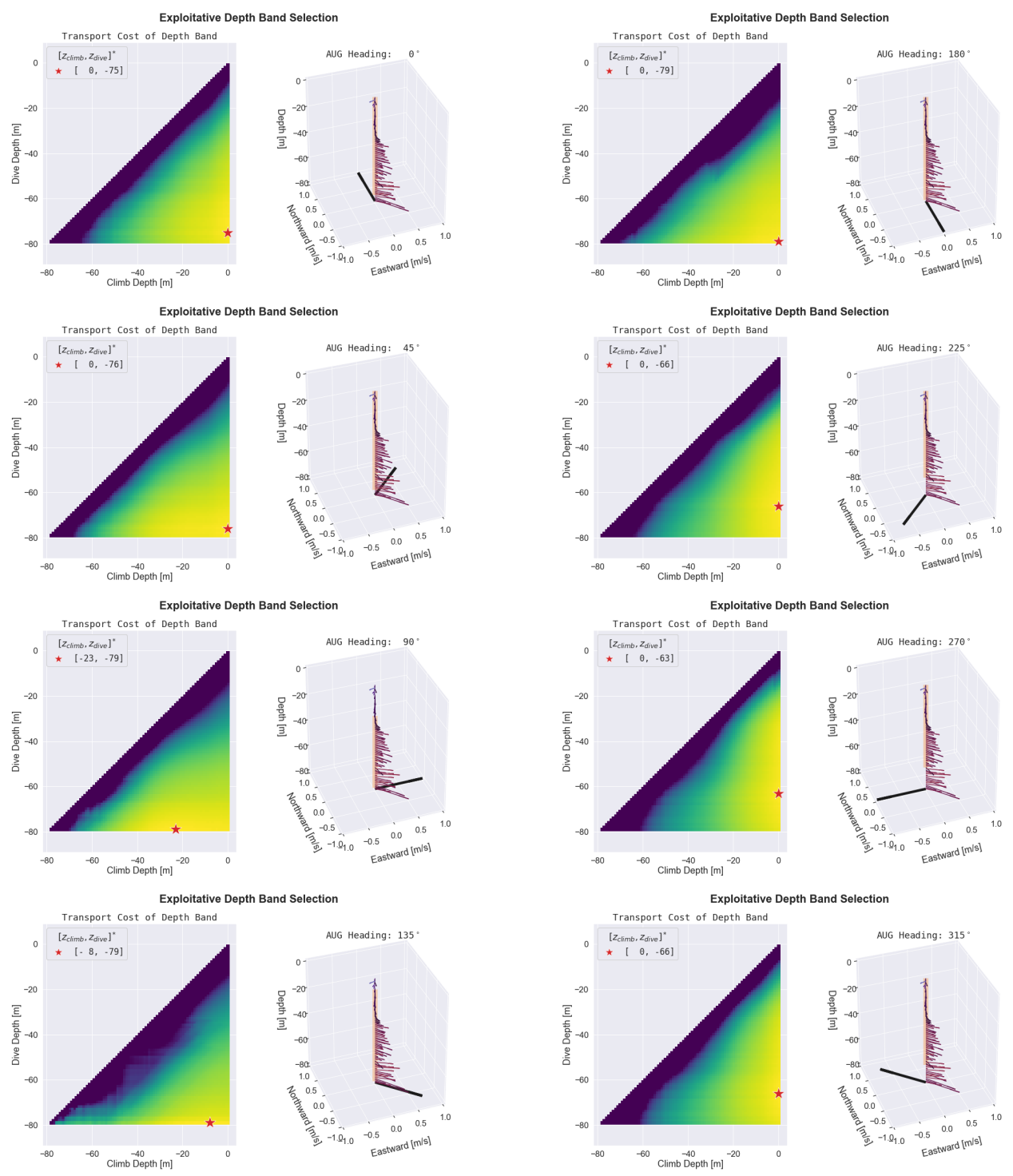

Figure 5-9: EDBS as applied to Dive F of the November 2019 research deployment in the Southern Aegean Sea. The EDBS method is applied at heading intervals of $45^{\circ}$. For each AUG heading considered, the left subplot shows the transport cost as a function of depth band, where the yellow color indicates low transport cost and the blue color indicates high transport cost. Similarly, each right subplot shows the water column velocities at $1 \mathrm{~m}$ depth intervals with the resulting optimal depth band highlighted in orange.

available water column (WC). The final column of the table shows the percent reduction in transport cost that the EDBS yields compared to the default full available water column approach. Importantly, for both dives, the EDBS method always performs as well or better compared to the default result. When velocity shear structure is present, as in Dive B, the EDBS method shows that transport cost can be reduced even if as little as $50 \%$ of the water 
column is utilized by the AUG. This result is rather remarkable because it contrasts with the commonly held belief that AUG efficiency is maximized when the full water column is utilized.

Although EDBS is designed to minimize transport cost and not AUG over-ground speed, metrics for the latter are also included in Table 5.3 because AUG speed over-ground has important implications for mission planning. Interestingly, the EDBS control policy tends to yield slightly lower over-ground speeds compared to the default WC alternative. This is because EDBS exploits favorable ocean currents, which in turn lead to slower over-ground speed when energy-optimal speed control is pursued. This phenomena is explored visually in Figure 5-10. Even though it is marginally reduced with EDBS, over-ground speed is consistent in magnitude regardless of heading direction and depth band control. This consistency is especially useful for scheduling and cooperating with multi-agent teams because the evolution of the AUG path is predictable regardless of the ocean current conditions. This idea of human robot interaction is explored further as a future work item in Chapter 6.

Since Dive B has strong velocity shear structure in the water column, the EDBS method is able to reap a greater percent reduction in transport cost compared to Dive F which has more uniform velocity structure throughout the water column. On average, the EDBS method achieves $6.36 \pm 5.76 \%$ reduction in transport cost compared to $\mathrm{WC}$ for Dive B, and the EDBS method achieves $1.43 \pm 1.22 \%$ reduction in transport cost compared to WC for Dive F.

Therefore, it is important to remember that the performance improvement attainable via applying the EDBS control policy is strongly dependent on environmental conditions. For a water column with fairly uniform structure, the EDBS policy will yield small or negligible improvements to transport cost compared to the default approach. However, when strong shear structure is present in the water column, the EDBS policy is able to achieve significant reductions in transport cost compared to the default approach. For example, for the water column profile shown in Dive B, the EDBS policy is able to achieve a transport cost reduction of $16.2 \%$. For similar shear structures as Dive B but with larger ocean current magnitudes, the performance improvement by EDBS will be even more pronounced.

Interestingly, EDBS tends to yield slightly slower speed over-ground than the default WC method, but overall, the AUG speed over ground is fairly consistent regardless of 


\begin{tabular}{|c||c|c|c||c|c|c||c|c|c|}
\hline \multicolumn{9}{|c|}{ Dive B from November 2019 Deployment in the Southern Aegean Sea } \\
\hline $\begin{array}{c}\text { AUG } \\
\begin{array}{c}\text { Heading } \\
\left.{ }^{\circ}\right]\end{array}\end{array}$ & $\begin{array}{c}\text { Optimal } \\
z_{\text {climb }} \\
{[\mathrm{m}]}\end{array}$ & $\begin{array}{c}\text { Optimal } \\
z_{\text {dive }} \\
{[\mathrm{m}]}\end{array}$ & $\begin{array}{c}\text { Utilized } \\
\text { WC } \\
{[\%]}\end{array}$ & $\begin{array}{c}\text { Avg. } \\
\text { WC } \\
{\left[\mathrm{m} \mathrm{s}^{-1}\right]}\end{array}$ & $\begin{array}{c}\text { Avg. } \\
\text { EDBS } \\
{\left[\mathrm{m} \mathrm{s}^{-1}\right]}\end{array}$ & $\begin{array}{c}\text { Avg. } \\
\text { Imp. } \\
{[\%]}\end{array}$ & $\begin{array}{c}f_{T C} \\
\text { WC } \\
{\left[\mathrm{J} \mathrm{m}^{-1}\right]}\end{array}$ & $\begin{array}{c}f_{T C} \\
\text { EDBS } \\
{\left[\mathrm{J} \mathrm{m}^{-1}\right]}\end{array}$ & $\begin{array}{c}f_{T C} \\
\text { Imp. } \\
{[\%]}\end{array}$ \\
\hline 0 & 39 & 79 & 50.6 & 0.734 & 0.734 & $\mathbf{0 . 0 2}$ & 7.12 & 6.92 & $\mathbf{2 . 8}$ \\
\hline 45 & 0 & 77 & 97.5 & 0.745 & 0.741 & $-\mathbf{0 . 5 2}$ & 8.40 & 8.39 & $\mathbf{0 . 1}$ \\
\hline 90 & 0 & 54 & 68.4 & 0.740 & 0.714 & $\mathbf{- 3 . 6 1}$ & 9.21 & 8.34 & $\mathbf{1 0 . 5}$ \\
\hline 135 & 0 & 50 & 63.3 & 0.726 & 0.694 & $\mathbf{- 4 . 4 8}$ & 9.40 & 8.09 & $\mathbf{1 6 . 2}$ \\
\hline 180 & 0 & 61 & 77.2 & 0.728 & 0.712 & $\mathbf{- 2 . 2 7}$ & 9.28 & 8.20 & $\mathbf{1 3 . 2}$ \\
\hline 225 & 0 & 63 & 79.7 & 0.745 & 0.719 & $\mathbf{- 3 . 5 2}$ & 8.75 & 8.34 & $\mathbf{4 . 9}$ \\
\hline 270 & 0 & 79 & 100.0 & 0.748 & 0.748 & $\mathbf{0 . 0 0}$ & 7.64 & 7.64 & $\mathbf{0 . 0}$ \\
\hline 315 & 32 & 79 & 59.5 & 0.694 & 0.671 & $\mathbf{- 3 . 3 2}$ & 6.38 & 6.18 & $\mathbf{3 . 2}$ \\
\hline
\end{tabular}

\begin{tabular}{|c||c|c|c||c|c|c||c|c|c|}
\hline \multicolumn{10}{|c|}{ Dive F from November 2019 Deployment in the Southern Aegean Sea } \\
\hline $\begin{array}{c}\text { AUG } \\
\begin{array}{c}\text { Heading } \\
{\left[{ }^{\circ}\right]}\end{array}\end{array}$ & $\begin{array}{c}\text { Optimal } \\
z_{\text {climb }} \\
{[\mathrm{m}]}\end{array}$ & $\begin{array}{c}\text { Optimal } \\
z_{\text {dive }} \\
{[\mathrm{m}]}\end{array}$ & $\begin{array}{c}\text { Utilized } \\
\text { WC } \\
{[\%]}\end{array}$ & $\begin{array}{c}\text { Avg. } \\
\text { WC } \\
{\left[\mathrm{m} \mathrm{s}^{-1}\right]}\end{array}$ & $\begin{array}{c}\text { Avg. } \\
\text { EDBS } \\
{\left[\mathrm{m} \mathrm{s}^{-1}\right]}\end{array}$ & $\begin{array}{c}\text { Avg. } \\
\text { Imp. } \\
{[\%]}\end{array}$ & $\begin{array}{c}f_{T C} \\
\text { WC } \\
{\left[\mathrm{J} \mathrm{m}^{-1}\right]}\end{array}$ & $\begin{array}{c}f_{T C} \\
\text { EDBS } \\
{\left[\mathrm{J} \mathrm{m}^{-1}\right]}\end{array}$ & $\begin{array}{c}f_{T C} \\
\text { Imp. } \\
{[\%]}\end{array}$ \\
\hline 0 & 0 & 75 & 94.9 & 0.719 & 0.717 & $-\mathbf{0 . 2 8}$ & 10.29 & 10.14 & $\mathbf{1 . 4 7}$ \\
\hline 45 & 0 & 76 & 96.2 & 0.724 & 0.722 & $-\mathbf{0 . 3 1}$ & 8.92 & 8.88 & $\mathbf{0 . 4 4}$ \\
\hline 90 & 23 & 79 & 70.9 & 0.720 & 0.716 & $-\mathbf{0 . 5 7}$ & 7.21 & 7.14 & $\mathbf{1 . 0 3}$ \\
\hline 135 & 8 & 79 & 89.9 & 0.714 & 0.713 & $-\mathbf{0 . 0 7}$ & 6.14 & 6.11 & $\mathbf{0 . 3 7}$ \\
\hline 180 & 0 & 79 & 100.0 & 0.728 & 0.728 & $\mathbf{0 . 0 0}$ & 6.64 & 6.64 & $\mathbf{0 . 0 0}$ \\
\hline 225 & 0 & 66 & 83.5 & 0.721 & 0.711 & $-\mathbf{1 . 2 9}$ & 7.79 & 7.68 & $\mathbf{1 . 4 1}$ \\
\hline 270 & 0 & 63 & 79.7 & 0.717 & 0.711 & $-\mathbf{0 . 9 0}$ & 9.32 & 8.99 & $\mathbf{3 . 6 6}$ \\
\hline 315 & 0 & 66 & 83.5 & 0.722 & 0.719 & $-\mathbf{0 . 3 9}$ & 10.42 & 10.11 & $\mathbf{3 . 0 9}$ \\
\hline
\end{tabular}

Table 5.3: Performance comparison between the EDBS method and the default behavior of utilizing the full available water column (WC). Water column data is taken from two dives from the November 2019 deployment in the Southern Aegean Sea. The dives are vertically scaled to $80 \mathrm{~m}$ for ease of comparison. Dive B has significant velocity shear structure in the water column while Dive F is more uniform throughout the water column. Performance is evaluated at $45^{\circ}$ heading intervals, where performance metrics include transport cost and average speed over ground.

heading direction and depth band behavior. This occurs because EDBS exploits favorable ocean currents in the water column, and in doing so, the energy optimal through-water speed given by AVC is sufficiently reduced that the over-ground AUG is actually lowered. The speed over-ground associated with energy-optimal speed control is further discussed in Figure 5-10.

\subsubsection{Mission Concept: Sea-ice Survey at the Chukchi Plateau}

Although the Slocum mission concept originally proposed by Stommel for AUGs over 30 years ago was focused on high-endurance physical oceanographic studies of the water column [Stommel, 1989], technological advancements in battery chemistry, hybrid thrust, and low-power computation and sensing, can enable AUG mission scenarios for high-endurance unattended missions in confined environments, such as sea-ice surveys in the Arctic. The 

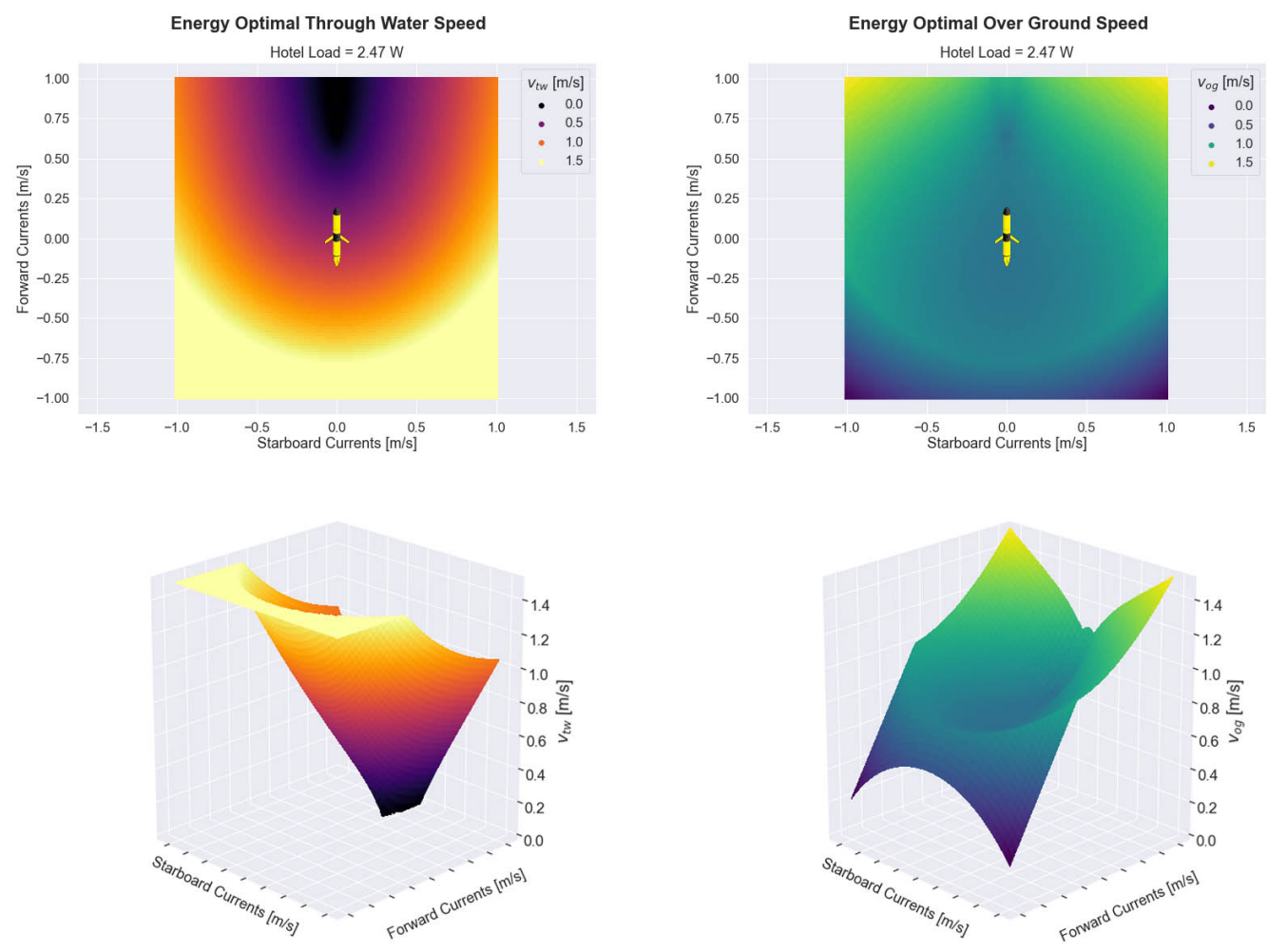

Figure 5-10: Energy-optimal through-water speed and over-ground speed for sea-ice survey using a reduced hotel load of $2.47 \mathrm{~W}$. The AUG over-ground speed is relatively constant across a fairly wide range of ocean current conditions, as indicated by the flat bowl shape in the 3D over-ground speed plot. Interestingly, the AVC control algorithm leads to a decrease in over-ground speed when small magnitude favorable ocean currents are encountered.

dangers of Arctic under-ice missions emphasizes on-board sensor interpretation, adaptive operation, and careful resource management. From an historical perspective, these constraints are not new. Mariners have faced similar requirements for millennia, and in many ways the AUG control policies that are proposed in this thesis are not unlike those devised centuries ago. In particular, opportunistic scavenging of low potential environmental energy has been understood at least since the first sail was affixed to a boat. The identification of ocean currents to improve velocity-made-good performance is clearly documented in Benjamin Franklin's analysis of sailing routes around the Atlantic Gulf Stream [Poupard and Franklin, 1786]. More recently, thermal AUGs [Webb et al., 2001] and wave AUGs [Hine et al., 2013] have demonstrated the ability to generate sufficient thrust exclusively from ocean thermal gradients and kinetic surface energy respectively, to propel robotic vehicles across entire marine basins. While the resource management policies that are proposed here 
are potentially useful in areas where other energy-scavenging platforms currently operate, they are more expansive in context. Specifically, these AUG resource management policies can provide a method for efficiently scavenging environmental energy while under sea-ice cover, where there is no opportunity to utilize wind, thermal gradients, or surface wave energy. Thus, with only modest modification to a legacy AUG design, it may be possible to greatly extend its range and observational capacity to enable low-cost, persistent, and unattended survey of marine polar regions.

With this scenario in mind it is possible to examine the possibility of a presently difficultto-impossible science mission: persistent unattended observation of the marginal ice zone (MIZ) and associated underlying water column temperature profile during seasonal sea-ice advance/retreat. During this time period the MIZ may migrate more than $40 \mathrm{~km} \mathrm{~d}^{-1}$, outpacing the speed of conventional buoyancy-driven gliders, potentially trapping and crushing them. If, for example, the marginal ice zone survey area were located at the Chukchi Plateau $\left[75.5^{\circ} \mathrm{N}, 164.0^{\circ} \mathrm{W}\right]$, and the AUG start and end point is located near Utqiagivik Alaska, which is the closest population center, the round-trip transit would be $913 \mathrm{~km}$. In this scenario, the transit path along the continental shelf margin has a typical water column depth of approximately $100 \mathrm{~m}$, enabling bottom lock DVL odometry, but the survey area includes regions with depths ranging beyond $1000 \mathrm{~m}$, necessitating use of an AUG capable of deep operation. In this hypothetical mission scenario the water column currents in the transit path are assumed to have shear flow characteristics commonly found at continental margins, equivalent to the shear flow encountered during Dive B shown in Figure 4-4, and that water column currents in the survey region include an additional velocity component in the deeper region of the water column generated by the anticyclonic Beaufort Gyre at $315^{\circ}$ azimuth with a $0.1 \mathrm{~m} \mathrm{~s}^{-1}$ horizontal velocity, as described by [Plueddemann et al., 1998]. Using these environmental state assumptions, the estimated mission duration and total range of scientific survey can be calculated for a conventional $1000 \mathrm{~m}$ buoyancy driven AUG and for a modified hybrid AUG utilizing various adaptive control policies.

Under the conditions of this mission scenario, conventional control using a buoyancy driven $1000 \mathrm{~m}$ Slocum AUG operating with a $26^{\circ}$ pitch angle would be unable to make the full round-trip transit, falling just short of a full round trip transit at $895 \mathrm{~km}$, without having initiated an ice survey at the study site. If the pitch angle is reduced to $12^{\circ}$, the buoyancy driven AUG would complete the round trip transit and only be able complete a 

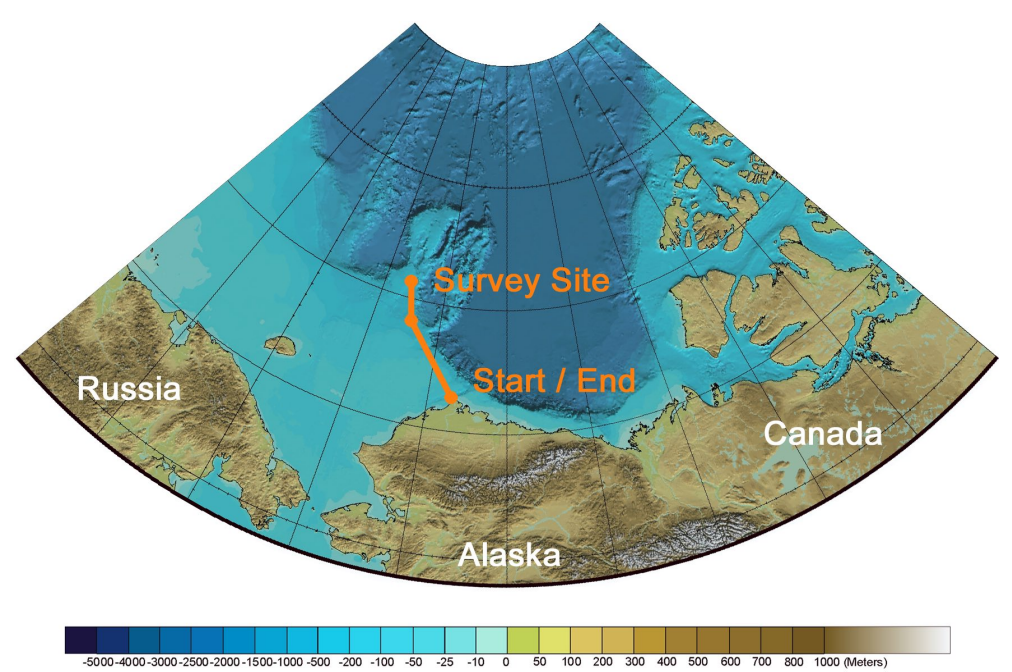

Figure 5-11: Overview of unattended AUG sea-ice survey mission scenario during the springsummer sea-ice retreat. For this mission, the AUG departs Utqiagivik, Alaska, transits $450 \mathrm{~km}$ to the survey site on the Chukchi Plateau, performs a sea-ice survey, and then transits $450 \mathrm{~km}$ back to Utqiagivik. The feasibility of this mission depends on the resource management of the AUG. Figure adapted from bathymetric map by [Jakobsson et al., 2012].

$285 \mathrm{~km}$ sea-ice survey. The addition of a hybrid thruster following the AVC control policy decreases transit time by almost a factor of 3 while increasing the total survey distance by over $200 \mathrm{~km}$. However, implementation of the additional adaptive control policies, ADC and EDBS, provide additional energy efficiency, enabling the AUG to increase the sea-ice survey by over an order of magnitude, as shown in Table 5.4.

\begin{tabular}{|c|c|c|c|c|c|c|c|c|}
\hline \multicolumn{4}{|c|}{ AUG Control Policy } & \multirow{2}{*}{$\begin{array}{l}\text { Transit } \\
\text { [d] }\end{array}$} & \multirow{2}{*}{$\begin{array}{l}\text { Transit } \\
{\left[\mathrm{J} \mathrm{m}^{-1}\right]}\end{array}$} & \multirow{2}{*}{$\begin{array}{c}\text { Survey } \\
\text { [d] }\end{array}$} & \multirow{2}{*}{$\begin{array}{c}\text { Survey } \\
{[\mathrm{km}]}\end{array}$} & \multirow{2}{*}{$\begin{array}{c}\text { Range Imp. } \\
{[\%]}\end{array}$} \\
\hline ID & Drive & Speed & Pitch & & & & & \\
\hline 1 & Buoyancy & Constant & $26^{\circ}$ & 28.6 & 40.2 & - & - & 0.0 \\
\hline 2 & Buoyancy & Constant & $12^{\circ}$ & 40.8 & 25.1 & 15.2 & 285 & 33.8 \\
\hline 3 & Hybrid & Constant & $12^{\circ}$ & 15.3 & 23.4 & 7.0 & 514 & 59.3 \\
\hline 4 & Hybrid & $\overline{\mathrm{AVC}}$ & $12^{\circ}$ & 14.3 & 22.5 & 7.1 & 558 & 64.3 \\
\hline 5 & $\mathrm{ADC}$ & AVC & $5^{\circ}$ & 16.6 & 7.4 & 44.4 & 2880 & 324 \\
\hline 6 & EDBS & AVC & $5^{\circ}$ & 16.8 & 6.6 & 43.9 & 3000 & 337 \\
\hline
\end{tabular}

Table 5.4: Performance analysis of six AUG control policies applied to a hypothetical sea-ice survey at the Chukchi Plateau. The survey mission includes $\approx 900 \mathrm{~km}$ round-trip transit, with remaining energy used for science data collection. The baseline AUG control policy is a buoyancy driven propulsion using $26^{\circ}$ pitch. Each successive control policy demonstrates continual performance improvement, where performance improvement is given by the increase in total range relative to the baseline control policy.

Although the EDBS is effectively a 1D path planner that optimizes for bathymetric 
constraints and water column currents along the vertical $z$-axis, the low-level energy resource management processes described here can be combined with higher-level $x y$-plane path planners that incorporate water current forecast modeling and risk assessment. Prior path planning work based on satellite observed surface water currents has demonstrated currentaugmented vehicle speed over-ground in excess of $1 \mathrm{~m} \mathrm{~s}^{-1}$ during transoceanic crossings [Ramos et al., 2018], improving energy efficiency while minimizing travel time.

\subsection{Summary and Future Work}

Looking toward the future of robotic under-ice survey, it is informative to consider that in addition to propulsion system inefficiencies, other AUG inefficiencies stem from limitations in fabrication processes which thereby constrain vehicle design. Although the resource management policies described here were applied to a legacy AUG design, they may, in principle be extended to other vehicle classes. As technological innovations in materials, manufacturing, and design advance, these policies should also be well-suited for next-generation designs that are more hydrodynamically efficient.

Under-ice survey of recently identified but distant ocean worlds of Europa and Enceladus is still the realm of science fiction, but may be within technological reach. For example, the NASA ICEE resource accommodation plan for a Europa surface lander limits the entire payload to just $32.7 \mathrm{~kg}, 1600 \mathrm{Wh}$, and 600 megabits for 20 days of stationary operations [Krajewski, 2018]. Survey below the estimated $10 \mathrm{~km}$ thick Europan ice sheet [Billings and Kattenhorn, 2005] using a mobile platform will almost certainly require decreased payload accommodation, suggesting that propulsion/hotel load balancing will result in a platform with a mass and power budget less than, or at most equivalent to, the AUG designs considered here. Assuming a mission cost in excess of $\$ 100 \mathrm{M}$ at present currency values, this translates to a Europan survey platform costing more than $\$ 1 \mathrm{M}$ per kg. Incorporating onboard sensing and control policies which can opportunistically identify and scavenge kinetic environmental energy sources as described here, along with thermal/chemical gradients could greatly reduce the required size of the power system, minimizing the vehicle system's mass while greatly extending mission life for under-ice exploration of these remote ocean worlds. Looking back toward polar Earth deployments, these innovations could provide an elegant approach for faster, more complete synoptic under-ice survey of polar marine 
environments without requiring scaling up in vehicle size, cost, and risk. As Jenkins et. al. aptly recognize, failure to adapt vehicle behavior in response to environmental state can lead to catastrophic losses of performance.

"Ocean currents can greatly enhance or degrade glide efficient. Failure to respond to those currents with appropriate adjustments to speed to fly can result in failure to exploit performance improvement offered up by Nature free of charge or can make even worse already catastrophic losses of performance."

[Jenkins et al., 2003]

The majority of this chapter focuses on the development of vehicle behaviors and policies that improve the energy efficiency of the AUG by adaptively taking advantage of environmental conditions, principally the water column currents. The set of policies derived in this Chapter are applicable to arbitrary AUG and AUV missions independent of the specific trajectory being pursued. However, further improvements to AUG energy efficiency can be achieved by exploiting water column current information during the mission planning process.

Similar to how the EDBS policy exploits velocity shear structure in the water column, waypoint selection and route planning routines can exploit three-dimensional structure in the ocean current vector field. If high-fidelity ocean current predictions are available, or if it is possible to perform ocean current simulations for the domain of interest, a rigorous partial differential equation (PDE) based approach that performs level set evolution can be used to determine energy-optimal or time-optimal paths for arbitrary ocean current conditions [Subramani and Lermusiaux, 2016]. However, for long endurance vehicles such as Polarsentinel, it is intractable to predict the ocean current field for the spatial and temporal scales that would be required. In the case of these long-endurance vehicles, it is possible to transmit local ocean current field to the AUG during surfacings so that the AUG can adaptively adjust its route plan [Ramos et al., 2018, Rao and Williams, 2009]. That said, neither of the these approaches include adaptive vehicle behaviors such as AVC and EDBS that actively minimize transport based on real-time estimates of environment state. Therefore, future research that combines energy optimized vehicle control policies based on real-time environment state with energy optimized mission planning based on high-fidelity ocean current predictions would be useful. 
Additionally, to build upon the idea of adaptive duty cycling of onboard instruments, further research can be done in the realm of active sampling. Similar to the active localization problem where the AUG seeks out sequences of poses that are conducive to limiting localization and navigation error, the active sampling problem is when the AUG seeks out sequences of poses and sensor control settings that are conducive to collecting science data. Importantly, active sampling is not simply a path planning problem of maximizing information gain along a trajectory, but active sampling also includes the control of AUG sensors. In the context of Arctic sea-ice survey, the AUG active sampling module seeks out routes that enable sea-ice observation and sends polling commands to the MSIS to make various sea-ice and sea-state characterizations. This process must be adaptive, reacting to vehicle and environment state as necessary. For example, if the AUG finds itself trapped underneath a collection of ice floes in the MIZ when trying to perform a surfacing maneuver, the AUG may need to make a series of sea-state measurements with the MSIS to back-out the wave propagation direction in hopes of discovering an area of ice-free fetch at the sea surface. Therefore, active sampling is not only helpful for energy efficiency purposes, but active sampling plays a critical role in risk assessment and onboard mission re-planning. Due to this coupling with mission planning, it is recommended that active sampling techniques are well-vetted via realistic simulation before pursuing sea-trials in the Arctic. 
This page intentionally left blank. 


\section{Chapter 6}

\section{Discussion and Future Work}

\subsection{Contributions}

This thesis aims to extend the autonomous capabilities of AUGs to enable basin-scale in-situ characterization of sea-ice in the Arctic. To support this central aim, this thesis includes three primary contributions: a hierarchical acoustics-based sea-ice characterization scheme to perform science data collection and assess environment risk, a multi-factor terrain-aided navigation method that leverages bathymetric features and active ocean current sensing to limit localization error, and a set of energy-optimal propulsive and hotel policies that react to evolving environmental conditions to improve AUG endurance. Together, these contributions allow modest-cost AUGs to survey the Arctic more persistently than ever before.

Although the contributions presented in this thesis are motivated by persistent basinscale sea-ice survey in the Arctic using an AUG, the benefits derived from these contributions are attainable for all AUVs, not just AUGs. Since AUVs are generally slow moving due to the inescapable drag penalty associated with high underwater speed, AUV dynamics can easily become dominated by ordinary ocean currents. To avoid catastrophic losses in performance due to being swept off-course or fighting against adverse currents, AUVs must be capable of adapting to their environment. Provided that appropriate sensors are available, this thesis describes how AUVs can actively sense and react to ocean currents in an energy-optimal manner. Though the need for energy efficiency is exacerbated by unattended operations in dangerous environments such as the Arctic, improving efficiency is ubiquitous for all forms of AUV operations. In addition to energy efficiency, navigation 
accuracy is another omnipresent requirement for all AUVs. By leveraging real-time ocean current and seafloor information, this thesis proposes a method for maintaining bounded navigation error without relying on acoustic beacons or periodic surfacing for GPS updates. As a result of devising an improved and self-contained approach for navigation, AUVs can pursue increasingly risky operations in pursuit of invaluable science data such as sea-ice survey in the Arctic. Therefore, by improving energy efficiency and navigation accuracy, this thesis allows AUVs in general to strive towards more exhaustive, more dangerous, and more scientifically uncharted missions.

\subsection{Future Work}

Since state estimation and control efficiency are central to the operation of autonomous systems, the contributions presented in this thesis can be extended in several key areas of AUG research. Such key areas include: human robot coordination, active sensing, active localization, execution monitoring, and verification and validation.

\section{Human Robot Coordination}

During AUG deployments, the vehicle does not interact with the human operator through physical interactions like a humanoid personal assistance robot. Instead, the AUG is given a mission plan to follow or science goals to explore, and the AUG is expected to intermittently report its progress back to the operator during the mission before the vehicle is later recovered by the operator. Thus, two principal considerations for human robot coordination emerge: communication and predictability. Intelligent communication allows the AUG to concisely convey important and actionable information during limited windows of opportunity when the AUG is at the sea surface. This thesis describes a method for transmitting compressed sea-ice classification information rather than raw acoustic data to reduce the time required for satellite communication. However, instead of communicating individual sea-ice classifications, it would be beneficial for the AUG to communicate the synoptic sea-ice map that has been computed during the mission so that the human operator can more readily adjust the mission plan if necessary. To accomplish this, previous efforts for semantic mapping and human-in-the-loop control can be leveraged [Jamieson et al., 2020]. Additionally, predictability of AUGs is important for scheduling coordinated actions such as 
launch and recover. Perhaps unexpectedly, the contributions of this thesis discovered that the energy optimal speed control of the AUG leads to fairly constant speed over-ground regardless of the ocean current conditions, as shown in Table 5.3 and Figure 5-10. As a future work item, it would be interesting to explore how predictable AUG behavior such as consistent over-ground speed can be exploited by multi-agent teams, such as human operators planning recovery operations or surface vessels supporting submerged vehicles.

\section{Active Sensing}

Active sensing tasks the robotic agent to adaptively plan sequences of control commands to satisfy some sensing objective; both sensor control variables and propulsive control variables are considered by the active sensing system. This thesis explores how sensor control variables, namely the control parameters of the mechanical scanning imaging sonar and the duty cycling of scientific equipment, could be actuated in response to sea-ice characterization objectives while maintaining energy efficiency. To further develop the active sensing of sea-ice surveying AUG, it would be valuable to include a science-driven hybrid mission and path planner that allows the AUG to sample the most interesting locations of the Arctic sea-ice cover [Smith et al., 2011, Ayton et al., 2019b, Ayton et al., 2019a]. For example, in the pursuit of characterizing the impact of emergent Arctic dynamics on annual sea-ice cover, it would be especially informative for the AUG to retrieve samples at the edge of the Marginal Ice Zone (MIZ), where the precise location of the MIZ boundary is poorly defined at the beginning of the mission.

\section{Active Localization}

Active localization is effectively a subset of active sensing, whereas the primary sensing objective of the AUG is minimizing navigation uncertainty. As discussed in Chapter 4, the navigation performance of the AUG is dependent on observable environmental features and path geometry. Path geometry is important for localization because relatively simple path geometry leads to more accurate ocean current estimation by the DVL-Odo module. Thus, the hybrid mission and path planning module can actively seek out trajectories that allow the AUG to localize itself with respect to the ground-truth bathymetric map [Censi, 2006, Häusler et al., 2013, He et al., 2011]. For example, the AUG path that leads to the best navigation solution may be significantly longer in terms of distance traveled than the 
direct path to the goal.

However, the path that leads to the best navigation solution may also be more dangerous in terms of the threat of collision with the seafloor. To account for the risk of collision, it may be possible to prescribe convex exclusion zones in the environment using the approximate convex decomposition (ACD) algorithm [Lien and Amato, 2004, Lien and Amato, 2007] that are constructed based on dynamic constraints of the vehicle such as maximum ascent angle. Then, these the active localization is tasked with planning a trajectory that maximizes navigation performance subject to the constraint that the trajectory does not coincide with any exclusion zones. The necessity for exclusion zones stems from the fact that several path planning modules rely on convex optimization for planning trajectories [Ono et al., 2013, Fernandez-Gonzalez et al., 2017].

In addition to trajectory planning for active localization, other sensor control parameters like AUG pitch and adaptive hotel load management can be optimized with respect to AUG localization. Therefore, active localization should be one of the prominent objectives considered by the multi-objective mission planning system, along with energy efficiency and science data collection.

\section{Execution Monitoring}

An execution monitoring system allows a robotic agent to determine when plan execution does not evolve as intended, thus allowing the agent to identify when mission replanning is necessary. The need for an execution monitoring system stems from uncertainties in the robot or uncertainties in the environment, where uncertainty may originate from missing information, unreliable resources, stochastic phenomena, or inherently vague concepts [Pettersson, 2005]. For example, the evolution of sea-ice cover in the Arctic is a stochastic process that is driven by ocean currents and atmospheric conditions, both of which are also uncertain processes. Therefore, the AUG mission planner has a limited ability to identify safe surfacing areas at the onset of the mission. Thus, an execution monitoring system is necessary to evaluate sea-ice classifications leading up to an intended surfacing location to decide whether or not the environment is sufficiently safe to surface. Execution monitoring onboard AUGs has demonstrated success in the past [Smith et al., 2012, Timmons et al., 2016], but future work must extend execution monitoring capabilities to handle domainspecific tasks such as surfacing from underneath ice cover. 


\section{Verification and Validation}

The analysis presented in this thesis was performed on real AUG and sensor data, but the analysis itself was performed during offline investigation. That said, to realize the potential of the contributions discussed in this thesis, the methods must be operationalized to run onboard the vehicle during field deployments. To do so, a sequence of verification and validation steps should be pursued, including simulation testing, bench testing, and field testing. Since sea-ice survey in the Arctic is inherently very dangerous for AUGs, it is important to make sure each software and hardware component is properly vetted before Arctic sea-trials. To do so, rigorous simulation testing can be performed to assure robustness, and analog sea-ice survey missions can be conducted locally where the threat of vehicle loss is significantly lower.

\subsection{Conclusion}

By enhancing the autonomous capabilities of AUGs via improvements to onboard environmental sensing, unattended navigation, and energy optimal control, it is possible to pursue increasingly daring missions such as basin-scale sea-ice surveys in the Arctic; chasing the vision shaped by Henry Stommel in 1989.

"I walk into our control room, with its panoply of views of the sea. There are updated global pictures from the remote sensors on satellites, there are evolving maps of subsurface variables, there are charts that show the position and status of all our Slocum scientific platforms, and I am satisfied that we are looking at the ocean more intensely and more deeply than anyone anywhere else."

[Stommel, 1989] 
This page intentionally left blank. 


\section{Bibliography}

[Anderson, 1950] Anderson, V. C. (1950). Sound scattering from a fluid sphere. The Journal of the Acoustical Society of America, 22(4):426-431.

[Anonsen and Hallingstad, 2006] Anonsen, K. B. and Hallingstad, O. (2006). Terrain aided underwater navigation using point mass and particle filters. In 2006 IEEE/ION Position, Location, And Navigation Symposium, pages 1027-1035. IEEE.

[Ardhuin et al., 2018] Ardhuin, F., Aksenov, Y., Benetazzo, A., Bertino, L., Brandt, P., Caubet, E., Chapron, B., Collard, F., Cravatte, S., Delouis, J.-M., et al. (2018). Measuring currents, ice drift, and waves from space: the sea surface kinematics multiscale monitoring (SKIM) concept. Ocean Science, 14(3):337-354.

[Armstrong et al., 2009] Armstrong, B., Pentzer, J., Odell, D., Bean, T., Canning, J., Pugsley, D., Frenzel, J., Anderson, M., and Edwards, D. (2009). Field measurement of surface ship magnetic signature using multiple AUVs. In OCEANS 2009, pages 1-9. IEEE.

[Armstrong et al., 2010] Armstrong, B., Wolbrecht, E., and Edwards, D. (2010). AUV navigation in the presence of a magnetic disturbance with an extended Kalman filter. In OCEANS'10 IEEE SYDNEY, pages 1-6. IEEE.

[Arnold and Medagoda, 2018] Arnold, S. and Medagoda, L. (2018). Robust model-aided inertial localization for autonomous underwater vehicles. In 2018 IEEE International Conference on Robotics and Automation (ICRA), pages 4889-4896.

[Arrigo et al., 2014] Arrigo, K. R., Perovich, D. K., Pickart, R. S., Brown, Z. W., van Dijken, G. L., Lowry, K. E., Mills, M. M., Palmer, M. A., Balch, W. M., Bates, N. R., et al. (2014). Phytoplankton blooms beneath the sea ice in the Chukchi Sea. Deep 
Sea Research Part II: Topical Studies in Oceanography, 105:1 - 16. The Phytoplankton Megabloom beneath Arctic Sea Ice: Results from the ICESCAPE Program.

[Asplin et al., 2012] Asplin, M. G., Galley, R., Barber, D. G., and Prinsenberg, S. (2012). Fracture of summer perennial sea ice by ocean swell as a result of Arctic storms. Journal of Geophysical Research: Oceans, 117(C6).

[Ayton et al., 2019a] Ayton, B., Timmons, E., Camilli, R., and Williams, B. (2019a). Adaptive science planning for the location of hydrocarbon seepage in the Costa Rica subduction zone. AGUFM, 2019:OS23C-1793.

[Ayton et al., 2019b] Ayton, B., Williams, B., and Camilli, R. (2019b). Measurement maximizing adaptive sampling with risk bounding functions. In Proceedings of the AAAI Conference on Artificial Intelligence, volume 33, pages 7511-7519.

[Ayton and Williams, 2018] Ayton, B. J. and Williams, B. C. (2018). Vulcan: a Monte Carlo algorithm for large chance constrained MDPS with risk bounding functions. arXiv preprint arXiv:1809.01220.

[Bar-Shalom et al., 2004] Bar-Shalom, Y., Li, X. R., and Kirubarajan, T. (2004). Estimation with applications to tracking and navigation: theory algorithms and software. John Wiley \& Sons.

[Barkby et al., 2009] Barkby, S., Williams, S., Pizarro, O., and Jakuba, M. (2009). An efficient approach to bathymetric SLAM. In 2009 IEEE/RSJ International Conference on Intelligent Robots and Systems, pages 219-224.

[Barkby et al., 2011] Barkby, S., Williams, S. B., Pizarro, O., and Jakuba, M. V. (2011). A featureless approach to efficient bathymetric SLAM using distributed particle mapping. Journal of Field Robotics, 28(1):19-39.

[Barnhart et al., 2014] Barnhart, K. R., Overeem, I., and Anderson, R. S. (2014). The effect of changing sea ice on the physical vulnerability of Arctic coasts. Cryosphere, 8(5).

[Bassett et al., 2020] Bassett, C., Lavery, A. C., Lyons, A. P., Wilkinson, J. P., and Maksym, T. (2020). Direct inference of first-year sea ice thickness using broadband acoustic backscattering. The Journal of the Acoustical Society of America, 147(2):824838. 
[Bassett et al., 2016] Bassett, C., Lavery, A. C., Maksym, T., and Wilkinson, J. P. (2016).

Broadband acoustic backscatter from crude oil under laboratory-grown sea ice. The Journal of the Acoustical Society of America, 140(4):2274-2287.

[Beattie and Geiger, 2017] Beattie, O. and Geiger, J. (2017). Frozen in time: the fate of the Franklin expedition. Greystone Books Ltd.

[Bellingham et al., 1995] Bellingham, J., Goudey, C., Consi, T., Bales, J., Atwood, D., Leonard, J., and Chryssostomidis, C. (1995). A second generation survey AUV. In Proceedings of IEEE Symposium on Autonomous Underwater Vehicle Technology (AUV'94), pages 148-155. IEEE.

[Bellingham et al., 2010] Bellingham, J. G., Zhang, Y., Kerwin, J. E., Erikson, J., Hobson, B., Kieft, B., Godin, M., McEwen, R., Hoover, T., Paul, J., et al. (2010). Efficient propulsion for the Tethys long-range autonomous underwater vehicle. In 2010 IEEE/OES Autonomous Underwater Vehicles, pages 1-7. IEEE.

[Benjamin et al., 2010] Benjamin, M. R., Schmidt, H., Newman, P. M., and Leonard, J. J. (2010). Nested autonomy for unmanned marine vehicles with MOOS-IvP. Journal of Field Robotics, 27(6):834-875.

[Billings and Kattenhorn, 2005] Billings, S. E. and Kattenhorn, S. A. (2005). The great thickness debate: Ice shell thickness models for Europa and comparisons with estimates based on flexure at ridges. Icarus, 177(2):397-412.

[Boeing, 2017] Boeing (2017). Echo Voyager product sheet. https://www.boeing. com/resources/boeingdotcom/defense/autonomous-systems/echo-voyager/echo_ voyager_product_sheet.pdf.

[Bradley et al., 1992] Bradley, A. M. et al. (1992). Low power navigation and control for long range autonomous underwater vehicles. In The Second International Offshore and Polar Engineering Conference. International Society of Offshore and Polar Engineers.

[Burgess et al., 2020] Burgess, G., Ventola, P., and Richard, C. (2020). Got ice? A statistical approach to marking sea ice and atmospheric conditions with a low-powered imaging sonar. 
[Butler and den Hertog, 1993] Butler, B. and den Hertog, V. (1993). Theseus: a cablelaying AUV. In Proceedings of OCEANS'93, pages I210-I213. IEEE.

[Carmack et al., 2016] Carmack, E. C., Yamamoto-Kawai, M., Haine, T. W., Bacon, S., Bluhm, B. A., Lique, C., Melling, H., Polyakov, I. V., Straneo, F., Timmermans, M.-L., et al. (2016). Freshwater and its role in the Arctic marine system: Sources, disposition, storage, export, and physical and biogeochemical consequences in the Arctic and global oceans. Journal of Geophysical Research: Biogeosciences, 121(3):675-717.

[Censi, 2006] Censi, A. (2006). Robot motion planning with uncertainty. Master's thesis, Università degli Studi di Roma.

[Christiansen et al., 2014] Christiansen, J. S., Mecklenburg, C. W., and Karamushko, O. V. (2014). Arctic marine fishes and their fisheries in light of global change. Global Change Biology, 20(2):352-359.

[Claus and Bachmayer, 2015] Claus, B. and Bachmayer, R. (2015). Terrain-aided navigation for an underwater glider. Journal of Field Robotics, 32(7):935-951.

[Claus and Bachmayer, 2016] Claus, B. and Bachmayer, R. (2016). Energy optimal depth control for long range underwater vehicles with applications to a hybrid underwater glider. Autonomous Robots, 40(7):1307-1320.

[Claus et al., 2012] Claus, B., Bachmayer, R., and Cooney, L. (2012). Analysis and development of a buoyancy-pitch based depth control algorithm for a hybrid underwater glider. In 2012 IEEE/OES Autonomous Underwater Vehicles (AUV), pages 1-6. IEEE.

[Cohen et al., 2018] Cohen, J., Pfeiffer, K., and Francis, J. A. (2018). Warm Arctic episodes linked with increased frequency of extreme winter weather in the united states. Nature communications, 9(1):1-12.

[Debevec et al., 2000] Debevec, P., Hawkins, T., Tchou, C., Duiker, H.-P., Sarokin, W., and Sagar, M. (2000). Acquiring the reflectance field of a human face. In Proceedings of the 27th annual conference on Computer graphics and interactive techniques, pages 145-156.

[Donovan, 2012] Donovan, G. T. (2012). Position error correction for an autonomous underwater vehicle inertial navigation system (INS) using a particle filter. IEEE Journal of Oceanic Engineering, 37(3):431-445. 
[Eustice et al., 2005] Eustice, R., Singh, H., Leonard, J. J., Walter, M. R., and Ballard, R. (2005). Visually navigating the RMS Titanic with SLAM information filters. In Robotics: Science and Systems, volume 2005, pages 57-64.

[Feltham, 2008] Feltham, D. L. (2008). Sea ice rheology. Annual Review of Fluid Mechanics, 40(1):91-112.

[Fernandez-Gonzalez et al., 2017] Fernandez-Gonzalez, E., Karpas, E., and Williams, B. (2017). Mixed discrete-continuous planning with convex optimization. In Thirty-First AAAI Conference on Artificial Intelligence.

[Francis and Skific, 2015] Francis, J. and Skific, N. (2015). Evidence linking rapid Arctic warming to mid-latitude weather patterns. Philosophical Transactions of the Royal Society A: Mathematical, Physical and Engineering Sciences, 373(2045):20140170.

[Furlong et al., 2012] Furlong, M. E., Paxton, D., Stevenson, P., Pebody, M., McPhail, S. D., and Perrett, J. (2012). Autosub long range: A long range deep diving AUV for ocean monitoring. In 2012 IEEE/OES Autonomous Underwater Vehicles (AUV), pages 1-7. IEEE.

[Griffin, 2013] Griffin, C. J. (2013). Operation Sunshine: The rhetoric of a Cold War technological spectacle. Rhetoric \& Public Affairs, 16(3):521-542.

[Haine et al., 2015] Haine, T. W., Curry, B., Gerdes, R., Hansen, E., Karcher, M., Lee, C., Rudels, B., Spreen, G., de Steur, L., Stewart, K. D., et al. (2015). Arctic freshwater export: Status, mechanisms, and prospects. Global and Planetary Change, 125:13-35.

[Häusler et al., 2013] Häusler, A. J., Saccon, A., Pascoal, A. M., Hauser, J., and Aguiar, A. P. (2013). Cooperative auv motion planning using terrain information. In 2013 MTS/IEEE OCEANS-Bergen, pages 1-10. IEEE.

[He et al., 2011] He, B., Zhang, H., Li, C., Zhang, S., Liang, Y., and Yan, T. (2011). Autonomous navigation for autonomous underwater vehicles based on information filters and active sensing. Sensors, 11(11):10958-10980.

[Heath et al., 2002] Heath, T. L. et al. (2002). The works of Archimedes. Courier Corporation. 
[Hegrenaes et al., 2009] Hegrenaes, O., Gade, K., Hagen, O. K., and Hagen, P. E. (2009). Underwater transponder positioning and navigation of autonomous underwater vehicles. In $O C E A N S$ 2009, pages 1-7. IEEE.

[Hine et al., 2013] Hine, R. G., Hine, D. L., Rizzi, J. D., Kiesow, K. A., Burcham, R., and Stutz, W. A. (2013). Wave power. US Patent App. 13/646,737.

[Horvat et al., 2016] Horvat, C., Tziperman, E., and Campin, J.-M. (2016). Interaction of sea ice floe size, ocean eddies, and sea ice melting. Geophysical Research Letters, 43(15):8083-8090.

[Houts et al., 2013] Houts, S. E., Dektor, S. G., and Rock, S. M. (2013). A robust framework for failure detection and recovery for terrain-relative navigation. Unmanned Untethered Submersible Technology.

[Hovdenak, 1935] Hovdenak, G. (1935). Amundsen's last journey. The Geographical Journal, 86:267-298.

[Hover et al., 2012] Hover, F. S., Eustice, R. M., Kim, A., Englot, B., Johannsson, H., Kaess, M., and Leonard, J. J. (2012). Advanced perception, navigation and planning for autonomous in-water ship hull inspection. The International Journal of Robotics Research, 31(12):1445-1464.

[Huang et al., 2010] Huang, A. S., Olson, E., and Moore, D. C. (2010). LCM: Lightweight communications and marshalling. In 2010 IEEE/RSJ International Conference on Intelligent Robots and Systems, pages 4057-4062. IEEE.

[Hunke and Lipscomb, 2015] Hunke, E. C. and Lipscomb, W. H. (2015). CICE: the Los Alamos Sea Ice Model Documentation and Software User's Manual. Los Alamos National Labratory, 5.1 edition.

[Jackson et al., 2012] Jackson, J. M., Williams, W. J., and Carmack, E. C. (2012). Winter sea-ice melt in the Canada Basin, Arctic Ocean. Geophysical Research Letters, 39(3).

[Jackson, 2016] Jackson, R. (2016). A morning in iceberg alley. Oceanus, 51(2):16. 
[Jakobsson et al., 2014] Jakobsson, M., Andreassen, K., Bjarnadóttir, L. R., Dove, D., Dowdeswell, J. A., England, J. H., Funder, S., Hogan, K., Ingólfsson, Ó., Jennings, A., et al. (2014). Arctic Ocean glacial history. Quaternary Science Reviews, 92:40-67.

[Jakobsson et al., 2012] Jakobsson, M., Mayer, L., Coakley, B., Dowdeswell, J. A., Forbes, S., Fridman, B., Hodnesdal, H., Noormets, R., Pedersen, R., Rebesco, M., Schenke, H. W., Zarayskaya, Y., Accettella, D., Armstrong, A., Anderson, R. M., Bienhoff, P., Camerlenghi, A., Church, I., Edwards, M., Gardner, J. V., Hall, J. K., Hell, B., Hestvik, O., Kristoffersen, Y., Marcussen, C., Mohammad, R., Mosher, D., Nghiem, S. V., Pedrosa, M. T., Travaglini, P. G., and Weatherall, P. (2012). The international bathymetric chart of the arctic ocean (IBCAO) version 3.0. Geophysical Research Letters, 39(12).

[Jakuba et al., 2015] Jakuba, M. V., Kinsey, J. C., Partan, J. W., and Webster, S. E. (2015). Feasibility of low-power one-way travel-time inverted ultra-short baseline navigation. In OCEANS 2015-MTS/IEEE Washington, pages 1-10. IEEE.

[Jakuba et al., 2008] Jakuba, M. V., Roman, C. N., Singh, H., Murphy, C., Kunz, C., Willis, C., Sato, T., and Sohn, R. A. (2008). Long-baseline acoustic navigation for under-ice autonomous underwater vehicle operations. Journal of Field Robotics, 25(1112):861-879.

[Jamieson et al., 2020] Jamieson, S., How, J. P., and Girdhar, Y. (2020). Active reward learning for co-robotic vision based exploration in bandwidth limited environments. arXiv preprint arXiv:2003.05016.

[Jeffries et al., 2013] Jeffries, M. O., Overland, J. E., and Perovich, D. K. (2013). The Arctic. Phys. Today, 66(10):35.

[Jenkins et al., 2003] Jenkins, S. A., Humphreys, D. E., Sherman, J., Osse, J., Jones, C., Leonard, N., Graver, J., Bachmayer, R., Clem, T., Carroll, P., et al. (2003). Underwater glider system study.

[Johnson-Roberson et al., 2010] Johnson-Roberson, M., Pizarro, O., Williams, S. B., and Mahon, I. (2010). Generation and visualization of large-scale three-dimensional reconstructions from underwater robotic surveys. Journal of Field Robotics, 27(1):21-51. 
[Jones et al., 2014] Jones, C., Allsup, B., and DeCollibus, C. (2014). Slocum glider: Expanding our understanding of the oceans. In 2014 Oceans-St. John's, pages 1-10. IEEE.

[Jurohi, 2008] Jurohi (2008). BSSRDF. https://commons.wikimedia.org/w/index.php? curid=4171648. Accessed: 2020-07-22.

[Kaiser et al., 2016] Kaiser, C. L., Yoerger, D. R., Kinsey, J. C., Kelley, S., Billings, A., Fujii, J., Suman, S., Jakuba, M., Berkowitz, Z., German, C. R., et al. (2016). The design and 200 day per year operation of the autonomous underwater vehicle Sentry. In 2016 IEEE/OES Autonomous Underwater Vehicles (AUV), pages 251-260. IEEE.

[Kaminski et al., 2010] Kaminski, C., Crees, T., Ferguson, J., Forrest, A., Williams, J., Hopkin, D., and Heard, G. (2010). 12 days under ice - an historic AUV deployment in the Canadian High Arctic. In 2010 IEEE/OES Autonomous Underwater Vehicles, pages $1-11$.

[Kepper et al., 2018] Kepper, J. H., Claus, B. C., and Kinsey, J. C. (2018). A navigation solution using a MEMS IMU, model-based dead-reckoning, and one-way-travel-time acoustic range measurements for autonomous underwater vehicles. IEEE Journal of Oceanic Engineering, 44(3):664-682.

[Kinsey et al., 2006] Kinsey, J. C., Eustice, R. M., and Whitcomb, L. L. (2006). A survey of underwater vehicle navigation: Recent advances and new challenges.

[Kinsey et al., 2008] Kinsey, J. C., Tivey, M. A., and Yoerger, D. R. (2008). Toward highspatial resolution gravity surveying of the mid-ocean ridges with autonomous underwater vehicles. In OCEANS 2008, pages 1-10. IEEE.

[Kinsey et al., 2013] Kinsey, J. C., Tivey, M. A., and Yoerger, D. R. (2013). Dynamics and navigation of autonomous underwater vehicles for submarine gravity surveying. Geophysics, 78(3):G55-G68.

[Kinsey and Whitcomb, 2004] Kinsey, J. C. and Whitcomb, L. L. (2004). Preliminary field experience with the DVLNAV integrated navigation system for oceanographic submersibles. Control Engineering Practice, 12(12):1541-1548. Invited Paper.

[Krajewski, 2018] Krajewski, J. (2018). Europa lander science payload draft proposal information package. https://nspires.nasaprs.com/ 
external/viewrepositorydocument/cmdocumentid=628861/solicitationId= \%7B17B73E96-6B65-FE78-5B63-84C804831035\%7D/viewSolicitationDocument=1/ Europa_PIP_Draft15v3.pdf. Accessed: 2020-06-30.

[Kukulya et al., 2016] Kukulya, A., Bellingham, J., Kaeli, J., Reddy, C., Godin, M., and Conmy, R. (2016). Development of a propeller driven long range autonomous underwater vehicle (LRAUV) for under-ice mapping of oil spills and environmental hazards: An Arctic domain center of awareness project (ADAC). In 2016 IEEE/OES Autonomous Underwater Vehicles (AUV), pages 95-100. IEEE.

[Kukulya et al., 2010] Kukulya, A., Plueddemann, A., Austin, T., Stokey, R., Purcell, M., Allen, B., Littlefield, R., Freitag, L., Koski, P., Gallimore, E., et al. (2010). Under-ice operations with a REMUS-100 AUV in the Arctic. In 2010 IEEE/OES Autonomous Underwater Vehicles, pages 1-8. IEEE.

[Kunz et al., 2009] Kunz, C., Murphy, C., Singh, H., Pontbriand, C., Sohn, R. A., Singh, S., Sato, T., Roman, C., Nakamura, K.-i., Jakuba, M., Eustice, R., Camilli, R., and Bailey, J. (2009). Toward extraplanetary under-ice exploration: Robotic steps in the Arctic. Journal of Field Robotics, 26(4):411-429.

[Kwok and Cunningham, 2015] Kwok, R. and Cunningham, G. F. (2015). Variability of Arctic sea ice thickness and volume from CryoSat-2. Philosophical Transactions of the Royal Society A: Mathematical, Physical and Engineering Sciences, 373(2045):20140157.

[Kwok et al., 2013] Kwok, R., Spreen, G., and Pang, S. (2013). Arctic sea ice circulation and drift speed: Decadal trends and ocean currents. Journal of Geophysical Research: Oceans, 118(5):2408-2425.

[Lee et al., 2012] Lee, C. M., Cole, S., Doble, M., Freitag, L., Hwang, P., Jayne, S., Jeffries, M., Krishfield, R., Maksym, T., and Maslowski, W. (2012). Marginal ice zone (MIZ) program: Science and experiment plan. Technical report, WASHINGTON UNIV SEATTLE APPLIED PHYSICS LAB.

[Lee et al., 2017] Lee, C. M., Thomson, J., Zone, M. I., and Teams, A. S. S. (2017). An autonomous approach to observing the seasonal ice zone in the western Arctic. Oceanography, 30(2):56-68. 
[Lehmenhecker and Wulff, 2013] Lehmenhecker, S. and Wulff, T. (2013). Flying drone for AUV under-ice missions. Sea Technology, 54(2):61-64.

[Leonard and Bahr, 2016] Leonard, J. J. and Bahr, A. (2016). Autonomous Underwater Vehicle Navigation, pages 341-358. Springer International Publishing, Cham.

[Lermusiaux et al., 2006] Lermusiaux, P. F., Chiu, C.-S., Gawarkiewicz, G. G., Abbot, P., Robinson, A. R., Miller, R. N., Haley, P. J., Leslie, W. G., Majumdar, S. J., Pang, A., et al. (2006). Quantifying uncertainties in ocean predictions. Technical report, HARVARD UNIV CAMBRIDGE MA.

[Lien and Amato, 2004] Lien, J.-M. and Amato, N. M. (2004). Approximate convex decomposition of polygons. In Proceedings of the twentieth annual symposium on Computational geometry, pages 17-26.

[Lien and Amato, 2007] Lien, J.-M. and Amato, N. M. (2007). Approximate convex decomposition of polyhedra. In Proceedings of the 2007 ACM symposium on Solid and physical modeling, pages 121-131.

[Lique et al., 2016] Lique, C., Holland, M. M., Dibike, Y. B., Lawrence, D. M., and Screen, J. A. (2016). Modeling the Arctic freshwater system and its integration in the global system: Lessons learned and future challenges. Journal of Geophysical Research: Biogeosciences, 121(3):540-566.

[Loranger, 2019] Loranger, S. C. (2019). Acoustic Detection and Quantification of Crude Oil. PhD thesis, University of New Hampshire.

[Lucido et al., 1996] Lucido, L., Opderbecke, J., Rigaud, V., Deriche, R., and Zhang, Z. (1996). A terrain referenced underwater positioning using sonar bathymetric profiles and multiscale analysis. In OCEANS 96 MTS/IEEE Conference Proceedings. The Coastal Ocean-Prospects for the 21st Century, volume 1, pages 417-421. IEEE.

[Lucieer et al., 2016] Lucieer, V., Nau, A. W., Forrest, A. L., and Hawes, I. (2016). Finescale sea ice structure characterized using underwater acoustic methods. Remote Sensing, $8(10)$. 
[Maksym, 2019] Maksym, T. (2019). Arctic and Antarctic sea ice change: Contrasts, commonalities, and causes. Annual Review of Marine Science, 11(1):187-213. PMID: 30216739 .

[Mallios et al., 2016] Mallios, A., Ridao, P., Ribas, D., Carreras, M., and Camilli, R. (2016). Toward autonomous exploration in confined underwater environments. Journal of Field Robotics, 33(7):994-1012.

[Mallios et al., 2014] Mallios, A., Ridao, P., Ribas, D., and Hernández, E. (2014). Scan matching SLAM in underwater environments. Autonomous Robots, 36(3):181-198.

[Markus et al., 2017] Markus, T., Neumann, T., Martino, A., Abdalati, W., Brunt, K., Csatho, B., Farrell, S., Fricker, H., Gardner, A., Harding, D., et al. (2017). The ice, cloud, and land elevation satellite-2 (ICESat-2): science requirements, concept, and implementation. Remote Sensing of Environment, 190:260-273.

[Medagoda et al., 2015] Medagoda, L., Kinsey, J. C., and Eilders, M. (2015). Autonomous underwater vehicle localization in a spatiotemporally varying water current field. In 2015 IEEE International Conference on Robotics and Automation (ICRA), pages 565-572.

[Medagoda et al., 2011] Medagoda, L., Williams, S. B., Pizarro, O., and Jakuba, M. V. (2011). Water column current profile aided localisation combined with view-based SLAM for autonomous underwater vehicle navigation. In 2011 IEEE International Conference on Robotics and Automation, pages 3048-3055.

[Mei et al., 2019] Mei, M. J., Maksym, T., Weissling, B., and Singh, H. (2019). Estimating early-winter Antarctic sea ice thickness from deformed ice morphology. The Cryosphere, 13(11):2915-2934.

[Melo and Matos, 2017] Melo, J. and Matos, A. (2017). Survey on advances on terrain based navigation for autonomous underwater vehicles. Ocean Engineering, 139:250-264.

[Meneghello et al., 2018] Meneghello, G., Marshall, J., Timmermans, M.-L., and Scott, J. (2018). Observations of seasonal upwelling and downwelling in the Beaufort Sea mediated by sea ice. Journal of Physical Oceanography, 48(4):795-805. 
[Miles et al., 2016] Miles, T., Lee, S. H., Wahlin, A., Ha, H. K., Kim, T. W., Assmann, K. M., and Schofield, O. (2016). Glider observations of the Dotson Ice Shelf outflow. Deep Sea Research Part II: Topical Studies in Oceanography, 123:16-29.

[Morgado et al., 2011] Morgado, M., Batista, P., Oliveira, P., and Silvestre, C. (2011). Position USBL/DVL sensor-based navigation filter in the presence of unknown ocean currents. Automatica, 47(12):2604-2614.

[Morgado et al., 2013] Morgado, M., Oliveira, P., and Silvestre, C. (2013). Tightly coupled ultrashort baseline and inertial navigation system for underwater vehicles: An experimental validation. Journal of Field Robotics, 30(1):142-170.

[NASA, 2020] NASA (2020). Mars 2020 Perseverance: Entry, descent, and landing technologies. https://mars.nasa.gov/mars2020/timeline/landing/ entry-descent-landing/\#Terrain-Relative-Navigation.

[Nicodemus, 1965] Nicodemus, F. E. (1965). Directional reflectance and emissivity of an opaque surface. Applied optics, 4(7):767-775.

[Nicolaus et al., 2012] Nicolaus, M., Katlein, C., Maslanik, J., and Hendricks, S. (2012). Changes in arctic sea ice result in increasing light transmittance and absorption. Geophysical Research Letters, 39(24).

[Ono et al., 2013] Ono, M., Williams, B. C., and Blackmore, L. (2013). Probabilistic planning for continuous dynamic systems under bounded risk. Journal of Artificial Intelligence Research, 46:511-577.

[Pasnani et al., 2018] Pasnani, P., Member, M. L. S. S., et al. (2018). Terrain-based localization and mapping for autonomous underwater vehicles using particle filters with marine gravity anomalies. IFAC-PapersOnLine, 51(29):354-359.

[Paull et al., 2014] Paull, L., Saeedi, S., Seto, M., and Li, H. (2014). AUV navigation and localization: A review. IEEE Journal of Oceanic Engineering, 39(1):131-149.

[Pettersson, 2005] Pettersson, O. (2005). Execution monitoring in robotics: A survey. Robotics and Autonomous Systems, 53(2):73-88. 
[Plueddemann et al., 1998] Plueddemann, A., Krishfield, R., Takizawa, T., Hatakeyama, K., and Honjo, S. (1998). Upper ocean velocities in the Beaufort Gyre. Geophysical Research Letters, 25(2):183-186.

[Poupard and Franklin, 1786] Poupard, J. and Franklin, B. (1786). A chart of the Gulf Stream. https://www.loc.gov/item/2004627238/. Accessed: 2020-07-18.

[Ramos et al., 2018] Ramos, A., García-Garrido, V., Mancho, A., Wiggins, S., Coca, J., Glenn, S., Schofield, O., Kohut, J., Aragon, D., Kerfoot, J., et al. (2018). Lagrangian coherent structure assisted path planning for transoceanic autonomous underwater vehicle missions. Scientific reports, 8(1):1-9.

[Rao and Williams, 2009] Rao, D. and Williams, S. (2009). Large-scale path planning for underwater gliders in ocean currents.

[Richter-Menge et al., 2006] Richter-Menge, J. A., Perovich, D. K., Elder, B. C., Claffey, K., Rigor, I., and Ortmeyer, M. (2006). Ice mass-balance buoys: a tool for measuring and attributing changes in the thickness of the Arctic sea-ice cover. Annals of Glaciology, $44: 205-210$.

[Rigby et al., 2006] Rigby, P., Pizarro, O., and Williams, S. B. (2006). Towards georeferenced AUV navigation through fusion of USBL and DVL measurements. In OCEANS 2006, pages 1-6. IEEE.

[Rypkema et al., 2017] Rypkema, N. R., Fischell, E. M., and Schmidt, H. (2017). One-way travel-time inverted ultra-short baseline localization for low-cost autonomous underwater vehicles. In 2017 IEEE International Conference on Robotics and Automation (ICRA), pages 4920-4926. IEEE.

[Sagalevitch, 2013] Sagalevitch, A. M. (2013). MIR submersibles historic dive under the ice dome at the geographical north pole: It has never happened before that small open window was overhead submersible crew instead of the immense ocean surface. In 2013 IEEE International Underwater Technology Symposium (UT), pages 1-6. IEEE.

[Salavasidis et al., 2019] Salavasidis, G., Munafò, A., Harris, C. A., Prampart, T., Templeton, R., Smart, M., Roper, D. T., Pebody, M., McPhail, S. D., Rogers, E., et al. (2019). 
Terrain-aided navigation for long-endurance and deep-rated autonomous underwater vehicles. Journal of Field Robotics, 36(2):447-474.

[Schaaf, 2020] Schaaf, C. (2020). BRDF/Albedo. https://modis-land.gsfc.nasa.gov/ brdf .html.

[Schwartz et al., 2002] Schwartz, E., Manseur, R., and Doty, K. (2002). Noncommensurate systems in robotics. International Journal of Robotics and Automation, 17(2):86-92.

[Schwarz and Weeks, 1977] Schwarz, J. and Weeks, W. (1977). Engineering properties of sea ice. Journal of Glaciology, 19(81):499-531.

[Schweiger et al., 2011] Schweiger, A., Lindsay, R., Zhang, J., Steele, M., Stern, H., and Kwok, R. (2011). Uncertainty in modeled Arctic sea ice volume. Journal of Geophysical Research: Oceans, 116(C8).

[Screen and Simmonds, 2010] Screen, J. A. and Simmonds, I. (2010). The central role of diminishing sea ice in recent Arctic temperature amplification. Nature, 464(7293):13341337.

[Serreze and Barry, 2011] Serreze, M. C. and Barry, R. G. (2011). Processes and impacts of Arctic amplification: A research synthesis. Global and Planetary Change, 77(1):85 96.

[Sherman et al., 2001] Sherman, J., Davis, R. E., Owens, W., and Valdes, J. (2001). The autonomous underwater glider: Spray. IEEE Journal of Oceanic Engineering, 26(4):437446 .

[Smith et al., 2012] Smith, R. N., Kelly, J., and Sukhatme, G. S. (2012). Towards improving mission execution for autonomous gliders with an ocean model and kalman filter. In 2012 IEEE International Conference on Robotics and Automation, pages 4870-4877. IEEE.

[Smith et al., 2011] Smith, R. N., Schwager, M., Smith, S. L., Jones, B. H., Rus, D., and Sukhatme, G. S. (2011). Persistent ocean monitoring with underwater gliders: Adapting sampling resolution. Journal of Field Robotics, 28(5):714-741. 
[Stammerjohn et al., 2012] Stammerjohn, S., Massom, R., Rind, D., and Martinson, D. (2012). Regions of rapid sea ice change: An inter-hemispheric seasonal comparison. Geophysical Research Letters, 39(6).

[Stommel, 1989] Stommel, H. (1989). The Slocum mission. Oceanography, 2(1):22-25.

[Stutters et al., 2008] Stutters, L., Liu, H., Tiltman, C., and Brown, D. J. (2008). Navigation technologies for autonomous underwater vehicles. IEEE Transactions on Systems, Man, and Cybernetics, Part C (Applications and Reviews), 38(4):581-589.

[Subramani and Lermusiaux, 2016] Subramani, D. N. and Lermusiaux, P. F. (2016). Energy-optimal path planning by stochastic dynamically orthogonal level-set optimization. Ocean Modelling, 100:57 - 77.

[Sullivan-Silva, 1989] Sullivan-Silva, K. (1989). Underwater acoustic scattering from spherical particulates and bubbles. Final Report Naval Underwater Systems Center.

[Sutherland and Gascard, 2016] Sutherland, P. and Gascard, J.-C. (2016). Airborne remote sensing of ocean wave directional wavenumber spectra in the marginal ice zone. Geophysical Research Letters, 43(10):5151-5159.

[Teixeira et al., 2015] Teixeira, F. C., Quintas, J., and Pascoal, A. (2015). AUV terrainaided navigation using a doppler velocity logger. IFAC-PapersOnLine, 48(2):137-142.

[Thomson and Rogers, 2014] Thomson, J. and Rogers, W. E. (2014). Swell and sea in the emerging Arctic Ocean. Geophysical Research Letters, 41(9):3136-3140.

[Timco and Frederking, 1996] Timco, G. and Frederking, R. (1996). A review of sea ice density. Cold regions science and technology, 24(1):1-6.

[Timmons et al., 2016] Timmons, E., Vaquero, T., Williams, B., and Camilli, R. (2016). Preliminary deployment of a risk-aware goal-directed executive on autonomous underwater glider. In PlanRob Workshop, ICAPS, volume 2016.

[Todd, 2017] Todd, A. L. (2017). Abandoned: the story of the Greely Arctic expedition, 1881-1884. Pickle Partners Publishing. 
[Todd et al., 2017] Todd, R. E., Rudnick, D. L., Sherman, J. T., Owens, W. B., and George, L. (2017). Absolute velocity estimates from autonomous underwater gliders equipped with doppler current profilers. Journal of Atmospheric and Oceanic Technology, 34(2):309-333.

[Toole et al., 2011] Toole, J. M., Krishfield, R. A., Timmermans, M.-L., and Proshutinsky, A. (2011). The ice-tethered profiler: Argo of the Arctic. Oceanography, 24(3):126-135.

[Van Uffelen et al., 2013] Van Uffelen, L. J., Nosal, E.-M., Howe, B. M., Carter, G. S., Worcester, P. F., Dzieciuch, M. A., Heaney, K. D., Campbell, R. L., and Cross, P. S. (2013). Estimating uncertainty in subsurface glider position using transmissions from fixed acoustic tomography sources. The Journal of the Acoustical Society of America, 134(4):3260-3271.

[Visbeck, 2002] Visbeck, M. (2002). Deep velocity profiling using lowered acoustic doppler current profilers: Bottom track and inverse solutions. Journal of atmospheric and oceanic technology, 19(5):794-807.

[Vogt et al., 2008] Vogt, C., Laihem, K., and Wiebusch, C. (2008). Speed of sound in bubble-free ice. The Journal of the Acoustical Society of America, 124(6):3613-3618.

[VonHaarberg, 2018] VonHaarberg (2018). BRDF mirror, BRDF glossy, BRDF diffuse. https://commons.wikimedia.org/w/index.php?curid=66095932. Accessed: 2020-0718.

[Wadhams, 2012] Wadhams, P. (2012). Arctic ice cover, ice thickness and tipping points. Ambio, 41(1):23-33.

[Wadhams and Doble, 2008] Wadhams, P. and Doble, M. J. (2008). Digital terrain mapping of the underside of sea ice from a small AUV. Geophysical Research Letters, 35(1).

[Wadhams and Horne, 1980] Wadhams, P. and Horne, R. (1980). An analysis of ice profiles obtained by submarine sonar in the Beaufort Sea. Journal of Glaciology, 25(93):401-424.

[Webb, 2006] Webb, D. C. (2006). Variable buoyancy device. Technical report, Teledyne Instruments, Inc. US Patent 7,096,814. 
[Webb et al., 2001] Webb, D. C., Simonetti, P. J., and Jones, C. P. (2001). SLOCUM: An underwater glider propelled by environmental energy. IEEE Journal of oceanic engineering, 26(4):447-452.

[Webster et al., 2015] Webster, S. E., Freitag, L. E., Lee, C. M., and Gobat, J. I. (2015). Towards real-time under-ice acoustic navigation at mesoscale ranges. In 2015 IEEE International Conference on Robotics and Automation (ICRA), pages 537-544. IEEE.

[Whitcomb et al., 1999] Whitcomb, L. L., Yoerger, D. R., and Singh, H. (1999). Combined doppler/LBL based navigation of underwater vehicles. In In: Proceedings of the the 11th International Symposium on Unmanned Untethered Submersible Technology.

[Williams et al., 2015] Williams, G., Maksym, T., Wilkinson, J., Kunz, C., Murphy, C., Kimball, P., and Singh, H. (2015). Thick and deformed Antarctic sea ice mapped with autonomous underwater vehicles. Nature Geoscience, 8(1):61-67.

[Willis, 2009] Willis, Z. (2009). The Scarlet Knight's trans-Atlantic challenge. Technical report, Rutgers University. http://rucool.marine.rutgers.edu/atlantic/.

[Yoerger et al., 2007] Yoerger, D. R., Jakuba, M., Bradley, A. M., and Bingham, B. (2007). Techniques for deep sea near bottom survey using an autonomous underwater vehicle. The International Journal of Robotics Research, 26(1):41-54.

[Zhang et al., 2013] Zhang, J., Lindsay, R., Schweiger, A., and Steele, M. (2013). The impact of an intense summer cyclone on 2012 Arctic sea ice retreat. Geophysical Research Letters, 40(4):720-726.

[Zhang et al., 2015] Zhang, J., Schweiger, A., Steele, M., and Stern, H. (2015). Sea ice floe size distribution in the marginal ice zone: Theory and numerical experiments. Journal of Geophysical Research: Oceans, 120(5):3484-3498.

[Zhou et al., 2019] Zhou, M., Bachmayer, R., and deYoung, B. (2019). Mapping the underside of an iceberg with a modified underwater glider. Journal of Field Robotics, 36(6):1102-1117.

[Zippel and Thomson, 2016] Zippel, S. and Thomson, J. (2016). Air-sea interactions in the marginal ice zone. Elementa: Science of the Anthropocene, 4:000095. 